\title{
Perceptual identification in normal ageing, dementia of the Alzheimer type, and depression
}

Citation for published version (APA):

Abbenhuis, M-A. (1995). Perceptual identification in normal ageing, dementia of the Alzheimer type, and depression. [Doctoral Thesis, Maastricht University]. Rijksuniversiteit Limburg.

https://doi.org/10.26481/dis.19950531ma

Document status and date:

Published: 01/01/1995

DOI:

10.26481/dis.19950531ma

Document Version:

Publisher's PDF, also known as Version of record

\section{Please check the document version of this publication:}

- A submitted manuscript is the version of the article upon submission and before peer-review. There can be important differences between the submitted version and the official published version of record.

People interested in the research are advised to contact the author for the final version of the publication, or visit the DOI to the publisher's website.

- The final author version and the galley proof are versions of the publication after peer review.

- The final published version features the final layout of the paper including the volume, issue and page numbers.

Link to publication

\footnotetext{
General rights rights.

- You may freely distribute the URL identifying the publication in the public portal. please follow below link for the End User Agreement:

www.umlib.nl/taverne-license

Take down policy

If you believe that this document breaches copyright please contact us at:

repository@maastrichtuniversity.nl

providing details and we will investigate your claim.
}

Copyright and moral rights for the publications made accessible in the public portal are retained by the authors and/or other copyright owners and it is a condition of accessing publications that users recognise and abide by the legal requirements associated with these

- Users may download and print one copy of any publication from the public portal for the purpose of private study or research.

- You may not further distribute the material or use it for any profit-making activity or commercial gain

If the publication is distributed under the terms of Article $25 \mathrm{fa}$ of the Dutch Copyright Act, indicated by the "Taverne" license above, 


\section{Perceptual identification in normal ageing, Dementia of the Alzheimer Type, and depression}

"Those who acknowledge their ignorance can learn something[..]".

Umberto Eco: Foucault's Pendulum 
Promotores:

Beoordelingscommissie: Prof. Dr. H.G. Schmidt (voorzitter)

Prof. Dr. B.G. Deelman (R.U. Groningen)

Dr. P.A.T.M. Eling (K.U. Nijmegen)

Prof. Dr. M.A. van den Hout

Prof. Dr. H.M. van Praag

\section{CIP-DATA KONINKLIJKE BIBLIOTHEEK, DEN HAAG}

Abbenhuis, Marie-Antoinette

Perceptual identification in normal ageing, dementia of the Alzheimer type, and depression / M.A. Abbenhuis. [S.I. : s.n.]

Proefschrift Aijksuniversiteit Limburg Maastricht. - Met lit. opg. - Met samenvatting in het Nederlands.

ISBN 90-9008190-9

NUGI 716

Trefw: geheugen / dementie / veroudering.

Printed by: Datawyse Maastricht / Krips Repro Meppel 


\section{Perceptual identification in normal ageing, Dementia of the Alzheimer Type, and depression}

\section{Proefschrift}

ter verkrijging van de graad van doctor aan de

Rijksuniversiteit Limburg, te Maastricht, op gezag van de Rector Magnificus, Prof. Mr. M.J. Cohen, volgens het besluit van het College van Dekanen, in het openbaar te verdedigen op woensdag, 31 mel 1995 om $16.00 \mathrm{uur}$

door

Marie-Antoinette Abbenhuis

geboren op 8 aprill 1957 te Uden 



\section{TABLE OF CONTENTS}

1.1 Normal ageing

1.2 Dementia of the Alzheimer Type (DAT) 5

1.3 Depression 6

1.4 Implicit and explicit measures of memory 8

1.5 Automatic and controlled memory processing 10

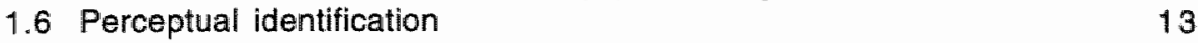

1.7 General outline of the present study 16

2.1 Episodic memory in dementia of the Alzheimer type and in normal ageing: Similar impairment in automatic processing (Experiment 1 and 2)

2.2 No difference in episodic memory performance between DAT patients, depressed patients, and normal elderly (Experiment 3)

3.1 Introduction

3.2 A comparison of DAT patients, depressed patients, and normal elderly on a perceptual identification task with words (Experiment 4),

3.3 Perceptual identification of words in six age-groups (Experiment 5). 43

3.4 Perceptual identification and recognition of words and nonwords in three age-groups (Experiment 6)

4.1 Introduction

4.2 Perceptual identification with visual, auditory, and no feedback in DAT patients and normal elderly (Experiment 7)

4.3 Perceptual identification with wisual, auditory, and without feedback in young, middle-aged, and elderly subjects (Experiment 8) 58

4.4 Discussion 
5.1 Introduction

5.2 A comparison of the performance of DAT patients and normal elderly subjects on perceptual identification after three different delays (Experiment 9)

5.3 A comparison of the performance of young, middle-aged, and elderly subjects on perceptual identification three different delays (Experiment 10)

6.1 Summary of the results of the present study $\quad 77$

6.2 Perceptual identification in normal and memory-impaired old adults 78

6.3 The contribution of semantic and episodic components in perceptual identification

6.4 Implicit memory as a description of structures or task manipulations 


\section{Chapter 1}

\section{General introduction}

"Home is our memony. The only true memory. For memory is our home".

Carios Fuentes: The Old Gringo.

Memory can be described as the capacity to retain information for later use. The matter of how the human organism retains information has been the subject of study of many scientists since numerous centuries (Yates, 1990). There is much discussion over which particular brain structures, or even whether particular brain structures are involved (Rosenfield, 1988), which processes act on the capacity to retain information, whether all information we perceive is retained, and if so, how it is stored, etc.

One of the main characteristics of memory research is that one can easily be overwhelmed by the wealth of theories and models trying to describe this intriguing phenomenon. Most models focus upon a particular aspect of memory, but untll now, no overall theory of memory, that is to say, a theory which can explain all manifestations of normal and amnesic memory is available. Some models emphasize particular aspects of memory, while other madels focus on other aspects. Hence, it is important to keep in mind that none of these models are mutually exclusive. They overlap considerably in the data they can explain. Also, the employed concepts are often not very clearly defined, or are modified as new data become avallable. This makes the theoretical muddle even greater.

It goes beyond the purpose of this study to describe in detail all the theories which have been developed up on the subject of how human memory functions. We will only describe three recent classifications, which seem to have heuristic value, although their explanatory merits have, as yet, to be ascertained.

The first classification (implicit vs. explicit) can be interpreted as a way to divide retrieval tasks in a practically useful way. The second classification (automatic vs. controlled) describes processes that act upon the to-be-remembered information at encoding. The third classification (episodic vs. semantic) can be taken as a description 
of types of information that are processed. We chose the first two distinctions because they provide a practical framework to describe the memory disturbances that accompany normal ageing, pathological ageing and the memory impairments that can result from depression. The distinction between episodic and semantic memory is described in relation to the task we used (l.e. perceptual identification).

All three models have been the subject of much discussion. We will try to clearly describe the pro's and con's of each model, how they relate to each other, and what the results of studies on the effect of ageing. DAT and depression within each framework are. We will further elucidate these problems in the next sections.

Aside from the necessity to clearly define the framework within which one tries to investigate memory, it is important to delineate the intent of the investigation. For example, the purpose may be a) to differentiate between normal and abnormal memory, b) to clinically discern particular amnesic patient groups, c) to get more insight into the nature of the memory deficits of specific groups, or d) to evaluate the effect of drugs on memory. Each approach calls for different experimental designs. One approach is not preferable over the other and they often go together by necessity.

For example, if the examination of memory serves a neuropsychological purpose, $(e . g$. discerning normal from pathological memory) the way one investigates memory is usually by using standardized tests which have proven to differentiate among normal and amnesic subjects. These standardized tests have been shown to serve their diagnostic usefulness, but have little or no theoretical value. Or, at least, interpretations concerning the nature of the underlying memory deficit are difficult because many factors contribute to the performance on these tests which may have little to do with memory functioning per se. In relation to the memory deficits in normal ageing, Grober, Buschke, Chrystal, Bang and Dresner (1988) differentiate between apparent and genuine memory deficits. They presume that normal ageing is accompanied by apparent memory deficits. These deficits are due to the use of inefficient strategies, impaired attention, reduced processing capacity etc. Impairments in these factors contribute to deficient performance on standardized memory tests, but do not reflect a true memory deficit.

Concerning the second possible objective (i.e. differentiating between amnesic syndromes of various etiology), reliance on standardized tests of memory is usualiy too crude. For example, Butters (1984) states that "[..] reliance on a single quantitative measure of memory (e.g. the MQ) for the assessment of amnesic syndromes may have as many limitations as does the utilization of an isolated score on a naming or a fluency test for the full description of aphasia" ( $p$. 33). Accordingly, Lezak (1983, p. 179) states that "In the long run most degenerative conditions become neuropsychologically indistinguishable".

Hence, using standardized tests may serve the clinical objective of discerning normal and abnormal memory performance, but to differentiate among the memory deficits of varlous patient groups may require another approach, namely the search for underlying factors that constitute the memory deficits of various amnesic syndromes.

There are several ways to investigate the nature of memory functioning (Mitchell, 1989). One way is to vary a certain aspect (for example, task instructions or depth of processing at encoding) and observe what effect this variation has on various memory tasks. In these kinds of studies mostly young normal individuals serve as experimental subjects. From these studies inferences are made about normal memory functioning. Another way is to study memory functioning of diverse experimental groups (for example, amnesic subjects and normal controls) of which it is known that they differ in memory functioning. However, if the second approach is taken it is necessary that the 
selection of the patient groups is done with close scrutiny:

Thus, it may be clear that meticulous clinical, or neuropsychological evaluation is a necessary prerequisite for more theoretical classifications of the diverse manifestations of memory. On the other hand, measures of memory originating from experimental psychology may provide useful instruments for clinical differentiations among amnesic patient groups, which do have some theoretical basis.

As mentioned above, the study of how normal memory functions usually involves young normal subjects. Studying abnormal memory mostly involves amnesic patients such as patients suffering from alcoholic Korsakoff syndrome or patients with a clearly circumscribed lesion as a consequence of traumatic brain damage. These patients exhibit deficits limited to the cognitive domain of memory, without extensive deficits in other domains. Studying memory functioning in these patients might eventually lead to a better insight into intact memory functioning.

However, in the last few decades much research has also focused on patients who suffer from some kind of degenerative brain disease such as dementia which may arise in the later decades of life. The proportion of aged adults is vastly growing, and with it the number of demented patients has also increased. One of the first and the main characteristics of dementia is an impairment in memory functioning. However, memory disturbances are not limited to the pathological ageing process of dementia. Other disorders are also reported to be accompanied by disruptions in memory performance, such as depression. The distinction between depression and dementia is often very difficult to make. Also, it is not clear at all what exactly constitutes the memory impairment caused by normal ageing (see Light, 1991, for a review), although elderly people very often complain about problems with remembering. Not knowing what the nature of the memory deficit of the normally aged is will render the practice of differential diagnosis especially troublesome. Moreover, a direct comparison between the outcomes of the diverse studies is often difficult to make because of different interpretations of the concepts involved, different procedures used, etc.

Therefore, we chose to study performance of normal young adults, normal old adults, demented patients and depressed patients on only one particular test (perceptual identification). The choice of our experimental groups was based upon the difficulties of differentiating the memory disturbances of normal ageing, DAT, and depression.

The original objective of the study presented in this thesis was to explore whether it was possible to use an instrument originating from experimental psychology for the differential diagnosis of DAT and depression. However, after the first two experiments it became clear that a different approach was called for. The results of the two experiments were contrary to what we expected based on what was reported by other investigators. We concluded that it was necessary to broaden the perspective of our study to the effects of normal ageing on memory.

Few studies have been done in which pathologically aged subjects are directly compared (i.e. using the same tests or procedures) with healthy elderly, as well as with young adults (but see: Graf \& Schacter, 1985; Nebes, Brady \& Huff, 1989). One of the current theories about DAT is that this disease is caused by an accelerated ageing process. To draw conclusions concerning a possible accelerated ageing effect in DAT one should obviousiy have an understanding of the effects of normal ageing. Hence, it is necessary to include a group of young subjects in the study of memory deficit of normal older adults and the various elderly patient populations.

The general outline of the present study can be described as follows: We used the perceptual identification procedure 1) to get more insight into the effects of normal 
ageing on memory 2) to investigate whether it was possiblle with the procedure we used to discern the memory impairments of DAT patients and normal elderly, 3) to investigate whether it was possible to differentiate between the memory impairments of DAT patients and elderly depressed patients.

First we will describe some general findings and theories concerning the nature of the memory deficits that accompany normal ageing. DAT and depression. Next, we will narrow down the study of the nature of the memory deficit in ageing: DAT and depression by describing two conceptual frameworks of memory processing (i.e. the distinction between implicit and explicit memory on the one hand, and the difference between automatic and controlled memory processes on the other hand), as well as the results of studies that thave been done within these two frameworks. And, subsequently, we will describe the perceptual identification task after which the relevant distinction between episodic and semantic memory will be discussed.

\subsection{NORMAL AGEING}

Much research on memory in recent years has concentrated on the memory impairments of old age. More than twenty years ago, Anders, Fozard and Lillyquist (1972) stated that the nature of the memory impairments in oider aduits was not clear. In her review of the recent literature, Light (1991) comes to a similar conclusion, namely that a sound explanation for the memory problems older adults experience is still not availlable.

Older adults often complain about memory problems in activities of daily living. In traditional memory tasks, the most consistent and striking impairments of elderly persons are found on recall and recognition tasks (Craik, 1977; Davis et al., 1990; Light, 1991; Mitchell, 1989; Mitchell, Hunt \& Smitt, 1986). The impairment of older adults on free-reciall tasks, however, is more severe than on recognition tasks.

The difference between older adilits' performance on recall and recognition tests led to the production deficiency hypothesis (Craik, 1977; Craik \& Simon, 1980). This hypothesis holds that elderly are less inclined to choose the appropriate self-initiated strategies at encoding and retrieval. This hypothesis is related to the assumption that older adults suffer from reduced attentional resources or capacity (see below) in that attentional resources or capacity are taxed by the use of strategies. Elderly subjects are inclined to encode information at a more superticial and less elaborate level. That they are, however, capable of more elaborate encoding is shown on tasks in which instructions induce, for example, the organization of the to be learned material into caltegories (Rankin \& Hinrichs, 1983).

This led Craik (1977) to the assumption that age-related impairments will disappear whenever retrieval conditions are optimal. Optimal retrieval conditions are those, which bear the greatest resemblance to the circumstances under which acquisition has taken place. So, as long as there is a considerable similarity between the processes involved at acquisition and retrieval, no age-related impairments should arise. Recognition of a stimulus requires less of retrieval processes, because the stimulus is presented again and serves in itself as a retrieval cue. In recall tasks, on the other hand, the to be remembered information is not avallable at the retrieval stage and an active, selfinitlated search strategy is necessary. The foregoing is used to explain the difference in recall and recognition performance of the elderly.

Another view is the general-slowing hypothesis. This position entails the idea that a general slowing of processing is responsible for the memory impairments in old age. 
Parallel to the physical and peripheral slow-down which can be seen in ageing, this view hypothesizes a general slowing of central processing, or simply a slowing of input and output processes (Balota \& Duchek, 1988). Which processes exactly are affected by sllowing is not clear. However, a general slowing of central and peripheral processes may exaggerate the observed impairments of older adults, especially on tasks that are paced or in which reaction time is a dependent variable.

A thitrd general hypothesis about the cause of age-related impairments is the assumption that elderly subjects suffer from reduced attentional resources (Craik \& Byrd, 1982; see for a review of this assumption: Salthouse, 1988). In this view it is conjectured that older adults have a reduced amount of activation available for attentional processes. So, in this view, the more effortful a task is (1.e. the more attentional processing is required) the greater the impairments older adults exhibit.

The evidence concerning these three hypotheses, however, is scarce. In her review, Light (1991) summarizes the evidence concerning these and related explanations for the memory impairments in old age. The only, more consistent findings are that, first, elderly subjects, in general are slower but that this only results in quantitative differences between the memory performances of older and young adults, and that, second, elderly subjects need greater contextual support both at encoding and retrieval. Likewise, Craik (1986) argues that ageing does not alter the kind of mental operations engaged in during a memory task, but it does alter the degree to whlch environmental support is needed at encoding and retrieval in order to compensate for an age-related decline in self-initiated activities.

\subsection{DEMENTIA OF THE ALZHEIMER TYPE (DAT)}

In the DSM III-R (1987) dementia is described as follows: "The essential feature is impairment in short- and long-term memory, associated with impairment in abstract thinking, impaired judgement, other disturbances of higher cortical function, or personality change" (p. 103). Dementia may result from a variety of causes. Primary degenerative dementia of the Alzheimer type (in this thesis we will refer to this disorder as Dementia of the Alzheimer Type: DAT) is described as involving [...] "a multifaceted loss of intellectual abilities, such as memory, judgement, abstract thought, and other higher cortical functions, and changes in personality and behavlour" (pp. 119-120). It is most often accompanied by pathological changes in the brain, such as pleques and tangles, widened cortical sulci and gyri and enlarged ventricles. The onset of DAT is insidious, and the course is progressively degenerating. A distinction is often made between an early onset (before age 65) and a late onset (after age 65). This boundary is rather arbitrarily chosen, and there is much discussion whether this distinction reflects two separable clinical pictures. As stated above, DAT is a progressively degenerating disease. The course of the disease is very idiosyncratic. There are, however, some general characteristics in the progression of the disease, which led Reisberg, Ferris, De Leon \& Crook (1982) to divide the course of DAT into five stages.

Diagnosis of DAT is extremely difficult. McKahn, Drachman, Folstein, Katzman, Price \& Stadian (1984) state that "Dementia is a diagnosis based on behaviour and cannot be determined by computerized tomography, electroencephalography, or other laboratory instruments, although specific causes of dementia may be identified by these means" (p. 940). They advised to divide the diagnostic criteria into possible, probable and definite DAT. For the diagnosis of probable DAT they advise a combination of 
neuropsychological, neurologic, psychiatric and laboratory tests. In vivo diagnosis is based upon the presence of dementia, and an insidious onset with a progressive degenerating course. Furthermore, all possible other causes for dementia ${ }_{\text {such }}$ as normal-pressure hydrocephalus, vitamin B 12 deficiency, hypothyroidism, etc. should be excluded by extensive laboratory testing, neurological and physical examinations. A diagnosis of possible DAT is appropriate when, in addition to the conditions that are necessary for a diagnosis of probable DAT, another disease which can be responsible for dementia is also present, or when the course is somewhat aberrant. A definite diagnosis is made when all the criteria for probable DAT are met in addition to postmortem histopathological confirmation of the disease. The patients who participated in the present study were diagnosed according to the criteria described in the DSM III (1980), and according to the criteria for probable DAT described by Mckahn et al. (1984). Furthermore we will designate the severity of the patlents according to the stages described by Reisberg et al. (1982).

Memory disturbances are among the first and most prominent features in DAT. Especially in an early stage of DAT it is sometimes very difficult to discriminate these patients" memory impairments from the memory disturbances of the normally aged, often described as "benign senescent forgetfuiness", especially when standardized tests of memory are used. For example, digit span is sometimes reported to be impaired in DAT (Kopelman, 1985; Nebes, Martin \& Horn, 1984) "and in other studies is found to be intact (Hart, 1985; Weingartner et al., 1981). This is also the case for elderly subjects. An excellent review of the memory impairments in DAT is given by Morris and Kopelman (1986).

Similarly, it is often difficult to discriminate DAT patients from other amnesic groups when using standardized tests. Shimamura, Salmon, Squire and Butters (1987) found similar impairments of patients suffering from alcoholic Korsakoff syndrome and DAT patients on a widely used standardized test: the Rey Auditory Verbal Learning Test. Free-Recall is always reported to be severely impaired in DAT (Nebes et al., 1984; Bondi \& Kaszniak, 1991). However, Heindel, Salmon, Shults, Walicke and Butters (1989) found that their DAT patients and their Huntington patients showed an equally impaired performance on a recall task compared to normal controls.

Recognition memory is often reported to be impaired in DAT (Nebes et al., 1984) but Heindel et al. (1989) also found a similarly impalied recognition performance in Huntington and DAT patients. Moss, Albert, Butters and Payne (1986) tested patients suffering from Huntington's disease, Korsakoff's syndrome, and DAT on a delayed recognition span test in which the cues to attend to were varied, such as spatial, colour, pattern, facial, and verbal cues. There was no difference between the three amnesic groups in the recognition of facial, spatial, colour or pattern stimuli: all patient groups performed worse than the controls. The only measure on which DAT patients performed worse than all other groups was the rate of forgetting: DAT patients forgot faster than all other groups.

\subsection{DEPRESSION}

One of the clinical pictures that complicates the diagnosis of DAT is depression in the elderly. On the one hand, patients with a Major Depressive Episode (DSM III-R, 1987) may complain of memory impairment, difficulty in thinking, and an overall reduction in intellectual abilities. They also may perform poorly on mental-status examination and neuropsychological testing. On the other hand, DAT patients while they are aware of 
the fact that their cognitive functioning is deteriorating, especially in the early stages, can present a depressed mood state; which can manifest itself in the presence of apathy, psychomotor retardation, welght loss and complaints of difficulty concentrating or memory loss. Lazarus, Newton, Cohler, Lesser and Schweon (1987) report that $40 \%$ of their patients with a presumptive diagnosis of DAT scored within the range of mild to severe depression on the Hamilton Depression Scale.

The DSM III-R describes as the essential fealure of Major Depression the occurrence of one or more Major Depressive Episodes. The main characteristic of a Major Depressive Episode is either depressed mood or loss of interest or pleasure in all, or almost all, activities, and assoclated symptoms, for a period of at least two weeks. Symptoms are relatively persistent and include appetite disturbance, change in weight, sleep disturbance, psychomotor agitation or retardation, decreased energy, feelings of worthlessness or excessive inappropriate guilt, difficulty thinking or concentrating, and recurrent thoughts of death, or suicidal ideation or attempts.

The percentage of misdiagnosis, that is patients diagnosed as suffering from DAT subsequently shown to be suffering from depression at follow up, can range from $5 \%$ to $15 \%$ (Feinberg \& Goodman, 1984), or even from 3\% to $60 \%$ with the majority of studies reporting a percentage misdiagnosis between $20 \%$ and $30 \%$ (Gurland \& Toner, 1983). As depression can be treated by the timely administration of appropriate medication, it may be clear that wrongly diagnosing a patient as suffering from DAT can have major consequences for the patient and his or her family.

As stated above, the behavioural pattern of depression and early DAT can be similar. Also, neuropsychological evaluation may show considerable similarities between the performance of DAT patients and patients with depression. Depressed patients are reported to score lower than controls but within the limits of performance of DAT patients on the Digit Symbol subtest of the WAIS (Hart, Kwentus, Wade \& Hamer, 1987), and the Visual Reproduction subtest of the WMS (Hart et al. "1987c). They also are reported to perform worse than controls, and sometimes as worse as DAT patients (Hart et al., 1987) on timed measures.

Some neuropsychological instruments do not differentiate between depressed elderly, mildly impaired DAT patients and normal elderly. Hart, Kwentus, Wade and Taylor (1988) found a simillar performance of these groups on a modified version of the Wisconsin Card Sorting Test. Only moderately impalred DAT patients performed worse than controls on all measures, but on some measures this test did not differentiate between the patient groups.

As for the impairments seen in DAT and normal ageing, recall and recognition are also reported to be impaired in depression (Kopelman, 1986b; O'Hara, Hinrichs, Kohout, Wallace \& Lemke, 1986; Watts \& Sharrock, 1987).

It will be clear from the aforementioned that standardized memory tests which use recall, recognition, or timed measures are not sufficient for clinical use in the practice of differential diagnosis, especially in an early stage of DAT.

In the following sections we will describe two models which can be useful for a more theoretically based differentiation between the memory impairments of DAT patients, depressed patients and normal elderly. 


\subsection{IMPLICIT AND EXPLICIT MEASURES OF MEMORY}

In a number of studies a clear dissociation was observed in amnesic patients between two types of memory tasks. If explicit reference at retrieval was made to the earlier studied material, amnesic patients showed a consistent deficit. However, if no reference was made to the original study task; these patients showed an equal to normal retention of the presented materials. This phenomenon has been addressed as "the difference between knowing how, and knowing that (Cohen \& Squire, 1980), procedural vs. declarative memory (Squire, 1982; Squire \& Cohen, 1984), or incidental vs. intentional learning (Jacoby, 1984).

However, these distinctions did not explain certain findings. For example, Moscovitch, Winocour and McLachlan (1986, exp. 1) tested young, old and older memory impaired subjects on reading of geometrically inverted text. After a variable delay they were offered old and new sentences. Reading time of the new sentences improved, indicating the acquisition of a general skill (i.e. procedural memory), but old sentences were consequently read most quickly, which suggested an item-specific priming effect, which could not be explained by the learning of a general procedure.

In 1985, Graf and Schacter introduced the distinction between implicit and explicit memory. Implicit memory is revealed when previous experiences facilitate performance on a task that does not require conscious or intentional recollection of those experiences. It is revealed in studies on savings in relearning, effects of subliminally encoded stimuli, and repetition or direct priming effects. Repetition priming tasks include: lexical decision, word identification, biased homophone spelling, and word stem or word fragment completion (Schacter, 1987). However, priming is not restricted to repetition or direct priming condittons (Shimamura, 1986). Amnesic patients exhibit normal priming even when cues are related semantically to previously learned material l.e. Cues that were never themselves presented for study (i.e. semantic priming task). Explicit memory is revealed when performance on a task requires conscious recollection of previous experiences (Schacter, 1987). They involve the classical recall, cued recall, and recognition tasks.

In amnesic patients there is a rather consistent difference between performance on implicit and explicit memory tasks, with implicit memory measures showing unimpaired performance, whille major deficits arise on explicit memory tasks (Graf, Squire \& Mandler, 1984; Jacoby \& Witherspoon, 1982).

Although the original description of the distinction between implicit and explicit memory seemed to imply of two separate memory systems (Schacter, 1985), Schacter (1987) states that this distinction should be taken as a description of a person's psychological experience at retrieval and not as a definition of two separate memory systems.

This is a rather vague description. It is not easy to see how a person's "psychological experience at retrieval" can be operationalized in a proper experimental design. Besides the fact that this definition is rather vague there are some practical problems in discerning implicit and explicit memory. For example, Graf et al. (1984) tested Korsakoff patients and normal controls on a word completion task with explicit, as well as implicit task instructions. Explicit instructions resulted in a better performance of the normal subjects compared to the patients. But periormance of normal subjects under the explicit condition was also better than their performance under the implicit condition. Therefore, Shimamura (1986) concluded that the control subjects profited from the explicit instructions, while the amnesic subjects did not. In other words, explicit reference at retrieval to the material originally studied provided some additional benefit 
for the control subjects. Finding differences between normal subjects and amnesic subjects on implicit memory tasks could be the result of normal subjects becoming aware that the material tested is related to the material studied. Schacter (1987) introduced the term "involuntary explicit memory" for this possibility. This factor cannot be excluded. Furthermore, informing subjects at the time of encoding that recollection of the studied material will be tested in a later stage, can increase the probability of involuntary explicit memory. Differences in implicit memory performance could be the result of normal subject's use of explicit memory strategies. Schacter (1987) proposes to present the implicit memory task as a filler task to reduce to a certain extent the possibility of involuntary explicit memory.

A difference between implicit and explicit performance not only arises in amnesic subjects but can also be observed in normal subjects under the appropriate experimental conditions. Several experimental variables differentially influence performance on implicit and explicit memory tasks in normal subjects. For example, levels of processing manipulations affect explicit performance, but not implicit performance (Jacoby \& Dallas, 1981); Giving explicit instructions in a word stem completion task, which turns it into a cued recall task, improved the number of stems completed after elaborative encoding compared to superficial encoding. Implicit memory instructions (i.e. no reference was made in the retrieval task to the previous studied words) did not show an effect of elaborateness of encoding (Graf \& Mandler, 1984); Modality shifts between study and test reduce priming, but not recognition (Jacoby \& Dallas, 1981; Graf, Shimamura \& Squire, 1985; Roediger \& Blaxton, 1987); Generating an item results in later recognition, but does not produce priming of that item (Jacoby, 1983b). And last, in a number of studies statistical independence has been demonstrated between implicit and explicit memory measures (Mitchell \& Brown, 1988; Parkin, Reid \& Russo, 1990; Perruchet \& Baveux, 1989; Squire, Shimamura \& Graf, 1985).

Implicit and Explicit Memory in Normal and Memory-Impaired Aged Subjects.

Performance of older adults on implicit tasks is generally believed to be intact. Preserved implicit memory has been found in a variety of tasks: Graf \& Schacter, 1985; Light, Singh \& Capps, 1986: word fragment completion; Light \& Singh, 1987: word stem completion and perceptual identification: Moscovitch, 1982: repeated reading of sentences).

For example, Graf and Schacter (1985) tested amnesic patients and their matched controls on word fragment completion. In this study, an additional control group consisting of young students was tested. They found no difference in the pattern of priming between amnesic patients, older adults and young adults.

As is found in normal old adults, a number of studies report an unimpaired performance of DAT patients on implicit memory tasks like semantic priming (Nebes et al. 1984), lexical decision in repetition priming (Moscovitch, 1982), or word stem completion (Miller, 1975; Morris, Wheatly \& Britton, 1983). There are, however, some studies that report impaired implicit memory in DAT patients. For example, Salmon, Shimamura, Butters and Smith (1988) tested DAT patients, amnesic Korsakoff patients, and patients suffering from Huntington's disease on a word stem completion task (lexical priming) and a task in which subjects had to produce a second word in response to a word of a previously studied pair (semantic priming) as measures of implicit memory. Explicit memory was tested by a recall and a recognition task. Explicit memory showed the normal pattern of impairments in the patient groups. However, DAT patients were selectively impaired on the two tasks of implicit memory. 
Also, Shimamura et al. (1987) tested mild to moderate, possible and probable DAT patients, Korsakoff patients, Huntington patients and their proper control groups on a word stem completion test and the Rey Auditory Verbal Learning Test. In the word stem completion test no reference was made to the subsequent memory test. At acquisition subjects had to judge how much they liked each word. DAT patients scored lower on the word stem completion test than any of the other groups. This deficit could not be related to severity of dementia for which their patient groups were matched. They attributed the word completion deficit of the DAT patients to a selective impalirment in memory activation rather than a global intellectual or cognitive decline. All patient groups showed a deficit on the standardized (explicit) test.

Bond and Kaszriak (1991) compared non-demented patients suffering from Parkinson's disease with DAT patients on various measures of implicil memory (Exp. 1: skill-learning and perceptual memory in a perceptual identification task with pictures, Exp. 2: word-stem completion, Exp. 3: pursulit rotor tracking (motor-skill learning), and explicit memory (Exp. 1: tree-recall, Exp. 2: cued recall). The DAT's were impaired compared to normal subjects and Parkinson patients on perceptual memory, and word-stem completion, but not on skill-learning, pursuit rotor tracking (considered to be a reflection of the learning of a specific procedure).

In summary, the most striking deficits in normal ageing, as well as in DAT, are found on explicit memory measures. Most often, the difference on explicit memory measures between normal and pathologically aged subjects is only quantitative. However, no difference arises betwoen various patient groups of differing etiology. On implicit memory measures, on the other hand, sometimes an impairment arises which is specific for the DAT patients. Thus, using an implicit memory task may provide a possible instrument for the purpose of differential diagnasis.

\subsection{AUTOMATIC AND CONTROLLED MEMORY PROCESSING}

In 1979, Hasher and Zacks published an article in which they moved away from more structural models of memory to a more process-orlented view on memory. They further explored the division in automatic and controlled memory processes. These two types of processing differed along dimensions of intention, awareness, susceptibility to inhibition, effects of stimulus lload, practice effects, etc. Hasher and Zacks" framework rests on two major assumptions: First that there is a continulum of attentional requirements among encoding processes; processes at the extremes of this continuum are refierred to as automatic and controlled. Second, that there is a variable capacity limit to attention and this limit interacts with encoding demands. With Kahneman (1973), they assume that attentional capacity varies both within and among individuals. The stimulus attributes that are encoded automatically are spatial location, time, frequency-of-occurrence, and word meaning. Effortful, or controlled processes are those that inwolve imagery, rehearsal, organization, and mnemonic techniques. They see the memory trace as an accumulation of attributes, of which some are encoded automatically, and some effortfully. However, they stress that whether or not a certain attribute is stored into memory a necessary condition is that the subject attends to the input; whether intentionally or not. They mention six characteristics of automatic processes: 1) they operate continually to encode attributes of whatever information is under the focus of attention 2) they can no longer be improved upon either by additional practice or by feedback about earlier performance 3) they do not require either intention, or awareness, and cannot be wilfully inhibited 4) they drain minimal 
amounts of energy from attentional capacity 5) knowledge gainod by automatic processing is accessible to consciousness, and 6) person can pay attention to incoming information that would otherwise be automatically encoded. Posner and Snyder (1975) also assume that they are not subject to interference, while Schneider and Shiffrin (1977) suggest that they can develop after a large amount of practice under the appropriate circumstances. In accordance with this last characteristic Hasher and Zacks assume that there are two types of automatic processes, to wit inherited and practised. To the first calegory belong operations that process frequency-of-occurrence, spatial location, and time of events. Processes that act on the meaning of words are reckoned to belong to the second category. The first category should be minimally influenced by differences in age, culture, education, early experience and intelligence because they are already present at birth: The second category of automatic processes develops after birth and can be influenced by factors such as early experience, intellect, education, and practice.

On the other hand, effortful, or controlled processes require conscious attention (Posner \& Snyder, 1975) and are open to strategies. These involve decisions, search, rehearsal and coding. However, on the role of consciousness in effortfull processes opinions differ. For example, Shiffrin and Schneider (1977) assumed that there are two types of effortful processes: veiled and accessible. Veiled processes occur quickly, and do not necessarily involve conscious awareness. Accessible processes are slow and can be perceived by the subject.

The model of Hasher and Zacks offers clear criteria to investigate which processes can be considered to be automatic. These criteria include 1) independence from intentional or incidental learning manipulations, 2) invariability under explicit instructions, or after practice, 3) they are insensitive to interference of concurrent processing, 4) states that alter attentional capacity (arousal, stress, depression, etc.) should have no effect, and last 5) the process of ageing does not affect automatic processing. All these criteria must be met to locate a process at the far end of automaticity.

How does the automatic/controlled distinction relate to implicit and explicit memory tasks? There are several parallels in the criteria for implicit memory and automatic processes, on the one hand, and explicit memory and controlled processing on the other hand. For example, intention to learn should not influence performance on implicit memory tasks, nor should it influence automatic processing. Implicit memory and automatic processing do not require awareness. There are, however, some differences. First, it should be stressed that Hasher and Zacks' model applies to the encoding stage, while Schacter's model applies to the retrleval stage. However, Hasher and Zacks (1979) state that their distinction may apply to the retrieval stage as well. Second, the implicit/explicit distinction was originally defined in terms of two separate systems, or might be defined in terms of task variables. The automatic/controlled distinction relates to different memory processes. Similarly, in accordance to the view of Craik (1977) who assumes that the same processes that act on the encoding stage may act on the retrieval stage as well, one can assume that the same principles that pertain to the encoding stage also have an effect on the retrieval stage. Hence. automatic processes can act at the encoding stage but also be of influence at the retrieval stage. Whether or not the task at hand is implicit does in itself not mean that either automatic or controlled processes dominate. But, the relative contribution of automatic processes under implicit retrieval conditions is likely to be greater than under explicit task instructions because conscious control is not necessary in implicit memory tasks. 
Automatic and Controlled Processing in Normal and Memory-Impaired Older Adults. The memory impaiments seen in old age are attribuled to a different use of strategies (Craik, 1977; Craik \& Rabinowitz, 1984), reduced attentionall resources (Puglisi, Park, Smith, \& Dudley, 1988; Park, Smith, Dudiey, \& La Fronza, 1989), or reduced capacity of working memory (Light, Zelinsky \& Moore, 1982; Salthouse, Mitchell, Skovronek \& Babcock, 1989). Stralegies, attention, and limited capacity are typical characteristics of controlled processing. It is on tasks in which a considerable demand is laid upon controlled processing and the limited capacity system of memory that the elderly show the greatest decrements (e.g. free-recall, divided attention, etc.). Thus ${ }_{n}$ it is assumed that controlled processes change with ageing while automatic processes (per definition) do not (Hasher \& Zacks, 1979; Howard, McAndrews \& Lasaga, 1981; but see: Howard, Shaw \& Heisey, 1986; Shimamura et al., 1987; Salmon et al., 1988).

Hasher and Zacks (1979; exp. 2) compared young and older adults on a frequencyof-occurrence task. Half of the subjects in each group were informed about the nature of the following retrieval task and half were not. Informing subjects about the nature of the task did not alter performance. This is taken as support for the notion that frequency-of-occurrence is encoded automatically. Results show that the judgement of older adults increased as actual frequency increased, although they did so at a slower rate than younger subjects.

Light and Singh (1987) compared young and older on repetition priming, word completion and perceptual identification. They found no differences in the size of the priming effects on either of these tasks, which they take as support for the notion that automatic activation processes are unimpaired in older adults. Light et all. (1986), also did not find age differences on a word fragment completion task. Similarly, Burke, White \& Diaz (1987) found no evidence for an age-related deficit in semantic priming.

Weingartner and his associates (Weingartner, Grafman, Boutelle, Kaye \& Martin, 1982; Weingartner, 1984; Strauss, Weingartner \& Thompson, 1985; Tariot \& Weingartner, 1986) view the automatic/controlled distinction as a useful framework for the purpose of differentiating the early memory impairments of DAT and those of depressed patients. They argue that depressed patients only show impaired performance on tasks that require effort, especially sustained effort (Silberman, Weingartner, Laraia, Byrnes \& Post, 1983) while DAT patients are both impaired on tasks that require effortful, as well as on tasks that mainly rely on automatic processing. For example, Strauss et al. (1985) tested subject suffering from Korsakoff's syndrome, Huntington's disease, and DAT on a frequency-of-occurrence memory task followed by a recall task. They presented words with a varying number of repetitions. Repetitions at encoding result in an increased recall in normal subjects, considered to be an effort-demanding process, while frequency-of-occurrence judgements are based upon automatic processing. The results show that, although performance of their Huntington and Korsakoff patients on the recall task was overall decreased, they showed evidence of benefit of repetitions. The DAT patients did not show such a benefit.

The assumption of Weingartner and his associates (Grafman et al., 1990; Newman, Weingartner, Smaliberg \& Calne, 1984) that depressed patients only show a deficit in controlled or effortful processing is in accordance with the Hasher and Zacks (1979) model. These authors state that attentional capacity interacts with arousal, mood ${ }_{n}$ and disease. Hasher and Zacks (1979; exp. 3) tested depressed and normal subjects on a frequency-of-occurrence task. The depressed subjects showed the same pattern of performance as the normal subjects did. They also tested depressed and normal subjects on a recognition task in which they presented a target and three distractors 
(Exp. 4). One of them was an associated word. They reasoned that if a target is presented for study associated words are automatically activated. This activation only persists for a short duration. If an associated word is still activated after a considerable period this must be as a consequence of a controlled process (e.g. rehearsal): On the other hand, recognition memory performance is presumed to be based on (automatically encoded) frequency tags. Thus, under the assumption that especially controlled processing is impaired in depression, they predicted that depressed and normal subjects would not differ in overall recognition performance (i.e. number correct), but that depressed subjects would be less inclined to falsely choose an associate (controlled process: rehearsal). This was confirmed by the results.

Hart et al. (1988) compared the performance of depressed patients and DAT patients using the Buschke selective reminding procedure. Although depressed patients showed an impairment on several measures (e.g. the use of imagery) on this task compared to normal controls, they did not differ on measures in which the use of effortful retrieval strategies is minimized (recall of recently presented items and recognition memory). DAT patients were impaired on all measures.

In the foregoing, we described two frameworks within which in recent years much research has been done. Although there is much ongoing discussion on the validity of these distinctions and their premisses they have a widely acknowledged heuristle value. The results of some studies within the implicit/explicit framework indicate that DAT patients show a deficit on implicit memory tasks not seen in normal older adults, nor in any other memory impaired patient groups. These results suggest that in order to differentiate between various amnesic patient groups implicit memory tasks could prove to be useful instruments. Similarly, it is suggested that only DAT patients exhibit decreased automatic processing, in contrast to normal elderly or depressed patients.

A suitable task to test whether implicit memory, which is predominantly based upon automatic memory processes is the perceptual identification task. In the next section we will describe the task and its theoretical basis.

\subsection{PERCEPTUAL IDENTIFICATION}

In most perceptual identification tasks stimull are presented for a very short duration. Subjects are required to identify these stimull. Memory is measured by comparing the probability (or speed) of identification of novel stimuli with that of earlier presented material. Repeated items generally have an advantage in identification over items which are presented for the first time and this advantage is called the facilitation effect or direct or repetition priming effects. We will use the former term to describe this effect. There is much discussion about what causes the facilitation effect. The relevant paradigm in this discussion is Tulving's distinction between episodic and semantic memory.

The Episodic/Semantic Distinction.

In 1972, Tulving introduced the distinction between episodic and semantic memory. As with the implicit/explicit distinction, it was originally meant to reflect two separable memory systems. Tulving (1983) defines episodic memory as an autobiographical record of unique episodes and events in an individual's experience, encoded and maintained in relation to a particular temporal-spatial context. Episodic memory is loosely organized and highly susceptible to forgetting. Its source is direct perception, or mere sensation. Its units are "events" ${ }^{*}$ with a definite affective tone. Access to 
episodic information is presumed to be deliberate and conscious.

Semantic memory, by contrast, is a relatively context-free thesaurus of organized knowledge regarding words, concepts and their associations, and rules for manipulating these symbols and concepts. It is highly organized and relatively permanent. For registration of information into the semantic memory system the content of that information should be understood and related to existing knowledge. Access to semantic memory is presumed to be automatic. It is considered to be devold of affective tone. Although most sclentists agree on the heuristic value of this dissociation, its theoretical value is subject to much discussion (See for example the peer comments on the article of Tulving, 1984 in the Behavioral and Brain Siciences). Tulving has made a variety of adaptations to his theory which seem rather ad-hoc. For example, originally the assumed the existence of two clearly dissociable memory systems (Tulving, 1972). In 1983, he suggested a larger framework in which lexical memory and procedural memory are also incorporated. Furthermore, he suggested in 1984 that episodic memory is embedded in the semantic system, and in 1985 he incorporated procedural memory into his model by suggesting that procedural memory underlies both semantic and episodic memory.

The discussion mainly concentrates upon the loose definition of the concepts of episodic and semantic memory which make them very difficult to be empirically tested (McKoon, Ratcliff \& Dell, 1986). Also, the empirical data are often inconclusive or even contradictory, or can be explained just as well within other theoretical frameworks in which a dissociation between episodic and semantic memory becomes superfluous (McClelland \& Rumelhart, 1985). Moreover, in terms of task characteristics it is often hard to label a task as purely episodic or semantic. McKoon et al. (1986) state that "labelling effects as semantic or episodic does not provide a theoretical explanation, but only a categorization (p. 295)".

Although there may be many theoretical problems with the episodic/semantic distinction, we also agree upon its heuristic value. We, for our part, only see the distinction as a categorization of the information that is processed. The amount of episodic or semantic processing that is involved in particular tasks is presumed to lie on a continuum (Burke et al., 1987) and depends upon the specific task characteristics. Tasks that measure retention of new information, for which no semantic representation can exist, are presumed to be purely episodic. Also, assuming that context in a very broad sense can never be exactly the same at two different points in time, tasks that reveal any kind of context-specificity, are considered to involve some amount of episodic information processing.

\section{Perceptual Identification and the Episodic/Semantic Distinction.}

In terms of the above mentioned characteristics of episodic memory (e.g. access is deliberate and conscious) recognition and recall should be considered to reflect episodic memory (note, that deliberate and conscious access are also the aspects of explicit memory, or controlled processes). However, the inherited automatic processes described in the model of Hasher and Zacks (1979; i.e. frequency-of-occurrence, spatial location, and time of events) are typically aspects which fall under the category of episodic memory. These are aspects of context of events and do not imply any knowledge about the content, or the meaning of events. Thus, some aspects which belong to the domailn of episodic memory can be processed automatically, in contrast to what Tulving (1983) proposes. However, originally, the facilitation effect was presumed to be based on semantic memory. For example, activation views hold that priming is based upon the temporary activation of pre-existing representations in 
memory (see, for example, Cermak, Talbot; Chandler \& Wolbarst, 1985; Graf and Mandler, 1984; Mandler, 1980; Morton, 1969; Shimamura, 1986).

Accordingly, multiple memory system views assume that explicit memory is per definition episodic, while priming tasks are based on semantic memory (see for a discussion of this notion McKoon et al., 1986; Roediger \& Blaxton, 1987; Schacter \& Tulving, 1982; Squire \& Cohen, 1984; Tulving 1983, 1986; Schacter 1987).

Nevertheless, there are several problems with the view that priming is purely based on semantic memory. For example, Tulving (1983) states that one of the characteristics of semantic knowledge is that it is not bound to a specific temporalspatial context. However, if the context between study and test is changed, priming effects are reduced (Richardson-Klavehn \& Bjork, 1988). Changing letter-case between first and second presentation resuits in reduced priming (Feustel, Shiffrin \& Salasoo, 1983; Jacoby \& Hayman, 1987; Jacoby \& Witherspoon, 1982) Similarly, a shift in modality between study and test reduces priming effects (Jacoby \& Dallas, 1981; Graf et all, 1985; Roediger \& Blaxton, 1987). It is difficult to imagine a separate general knowledge system existing within each modality. Also, if nonwords or pseudowords are used as stimuli normal subjects show priming. However, nonwords do not have a preexisting representation in semantic memory (Cermak et al., 1985; Forster \& Davis, 1984; Johnston, Dark \& Jacoby, 1985; McKoon \& Ratcliff, 1986; Schacter \& Graf, $1986 \mathrm{a}, \mathrm{b})$.

In contrast to the view that priming phenomena are solely based on the activation of pre-existing memory are the processing views of priming. Within these views it is argued that implicit measures of memory can also rely on newly established episodic representations (Craik, 1983; Jacoby, 1983a,b; McKoon \& Ratcliff, 1986; Moscovitch et al., 1986; Witherspoon \& Moscovitch, 1989). One argument for this notion is, for example, that it is difficult to see how a single presentation of words which have been encountered many times can have such large and long lasting effects on perceptual identification performance.

A third view is represented in the assumption that episodic and semantic information processes interact, while carrying out a memory task (Feustel et al., 1983; Salasoo, Shiffrin \& Feustel, 1985). However, a practical problem is that it is difficult to separate the relative contribution of semantic or episodic activation on priming tasks.

In summary, our choice for a perceptual identification task was stipulated by first, the nature of the facilitation effect which is presumed to be based upon automatic processing. Advantages of a test which tackles this type of prociessing are the following:

1. it is, per definition, supposed to be independent of age-related factors, or factors that alter attentional capacity, such as arousal, stress, or depression.

2. It is presumed to be independent of factors such as intelligence or educatlonal background.

Education has changed a lot since the beginning of the century, and sometimes age differences in memory performance are considered to be a result of this change (Light, 1991). And second, because it is an implicit memory task which offers an additional advantage in that it's instructions are very simple.

Comprehension is often reported to be impaired in DAT. The possibility of deflicits in comprehension calls for certain restrictions on test instructions. If subjects cannot comprehend the sometimes complex instructions of traditional memory tasks, it is obvious that impaired performance on that task cannot be ascribed to a memory deficit. The instructions of the perceptual identification task only involves reading the words aloud that are presented on the screen. Thus, there is little to comprehend so 
that performance of DAT patients will not be attenuated by deficits in comprehension. Moreover, even if no instructions are given, subjects spontaneously engage in reading aloud the words presented on the screen.

Additionally, often disturbances in language are reported in DAT (see for a review: Hart, 1988). It is repeatedly reported, for example, that naming performance is impaired in DAT. This can obscure the results of many memory tests; in which it is necessary to name objects, pictures etc. However, reading words aloud is preserved unitil the very late stages of DAT, as long as no reference is needed to the meaning of words (Hart, 1988). Knowing the meaning of words is irrelevant for an identification response.

\subsection{GENERAL OUTLINE OF THE PRESENT STUDY}

At the outset of the study, we expected the following results concerning perceptual identification performance:

1. There will be a difference in facilitation between DAT patients and normal elderly. This first hypothesis ensued from the results of Weingartner and his associates concerning a deficit in automatic processing of DAT patients.

2. Depressed patients will exhibit an unimpaired performance compared to normal elderly, but will show a greater amount of facilitation than DAT patients. The second hypothesis was based upon the presumptions of the Hasher and Zacks' (1979) model and the results they obtained in their depressed sample.

In our first experiment (Chapter 2, exp. 1) we compared moderately-to-severely impaired DAT patients with a sample of normal elderly. In contrast to what we expected, DAT patients performed equally well as healthy older adults. However, the facilitation effect we obtained in both groups was rather low compared to what Jacoby (1983 a,b) found in normal young subjects. This led us to the second experiment (Chapter 2, exp. 2), in which we explored the age-independence criterion of automatic processing. Again in contrast to what we expected, we found an age-related deficit in the facilitation effect.

Parallel to the beforementioned experiments we compared performance of mildy-to. moderately impaired DAT patients, depressed patients and a control group (Chapter 2, exp.3). We obtained similar results as in our first experiment, l.e. no difference botween DAT patients and controls. Moreover, the sample of depressed patients did not differ from the other two groups.

As a result of these outcomes we changed the emphasis of our study to the agerelated impairments we observed. However, all experiments were done with samples of DAT patients as well to explore whether factors that influenced age-related impairments would differentially affect the performance of our DAT patients.

In Chapter 3 (exp. 4), we first tested the hypothesis whether the fallure to find an effect of DAT on the facilitation effect was perhaps due to a floor-effect. Therefore, we changed the mode of feedback (auditory vs. visual) to increase the range of facilitation. Again, DAT patients, depressed subjects en controls did not differ. In the next experiment (Chapter 3 , exp. 5), we to determine the age at which a decrease in facilitation would arise in normal elderly. We used the same task (i.e. with visual feedback) as in the previous experiment and tested six age groups, ranging from 20 to 80 years. Surprisingly, no age-related impairment was found. Obviously, the difference in 
perceptual identification tasks we used in the experiments described in Chapter 2 and those described in Chapter 3, was crucial for finding an age-related deficit or not.

To explore whether finding an age-related impairment was due to a dieficit in episodic memory processing per se, we performed an experiment (Chapter 3 , exp. 6) in which nonwords were used as stimuli and tested three age groups. No difference was found on the facilitation of nonwords.

Because we not only changed mode of feedback but also changed the apparatus we replicated the foregoing experiments. The results are described in Chapter 4. In addition to testing subjects on perceptuall identification with auditory, as well as with visual feedback, we also tested subjects on a condition without feedback. This was done to explicitly determine the relative contribution of visual or auditory feedback on the facilitation effect.

The results concerning DAT (Chapter 4, exp. 7) were similar to our previous experiments. In the next experiment (Chapter 4, exp. B) we found the following: The results of the experiments described in Chapter 2 and 3 were replicated (i.e. an agerelated impairment on perceptual identification with auditory feedback, but not on perceptual identification with visual feedback). However, in the condition without feedback neither group (except the midole-aged) showed a notable facilitation effect. Clearly, feedback was a crucial factor in the manifestation of the facilitation effect, and varying the mode of feedback had differential effects upon young and elderly subjects.

In Chapter 5 (exp. 9 and 10) we further investigated the nature of the facilitation effect by changing the procedure altogether, and thus rendering feedback an irrelevant factor. These experiments were also done to determine the persistence of the facilitation effect by testing at three different intervals, and to explore whether our experimental groups would exhibit differences in the persistence of the facilitation effect.

This procedure again resulted in equal performance of DAT patients and controls, as well as in an age-dependent impairment in the amount of facilitation at the first two intervals. However, neither the older adults" facilitation effect, nor that of the DAT patients did deteriorate at a faster rate than that of the young subjects.

In Chapter 6 the general conclusions are discussed, as well as their consequences for the paradigm of implicit memory and the concept of automatic processing. 


\section{Chapter 2}

\section{Basic experiments}

"He looked at me and there was nobody home behind his eyes".

Patricia D. Cornwell: Body of Evidence.

\subsection{EPISODIC MEMORY IN DEMENTIA OF THE ALZHEIMER TYPE AND IN NORMAL AGEING: SIMILAR IMPAIRMENT IN AUTOMATIC PROCESSING (EXPERIMENTS 1 AND 2)}

\section{INTRODUCTION}

Dementia of the Alzheimer's Type (DAT) is generally defined as a clinical syndrome characterized by a global deterioration of cognitive functioning. Although the course of the disease is very idiosyncratic memory deficits develop very early on. However, disturbances in memory are in no way specific to DAT. For example, depressed patients and patients suffering from Korsakoff's syndrome also exhibit deficits on traditional recall and recognition tasks (Ellis, Thomas \& Rodriguez, 1984; Martone, Butters, Payne, Becker \& Sax, 1984).

As problems with memory are among the first symptoms, developing very early in the course of the disease, it is not surprising that much of the research of recent years has been focused on this topic. The use of research in this area may be that studying abnormal memory performance may render new insights about normal, healthy memory functioning. In order to determine the specific nature of the memory deficit in DAT or other disorders, it is necessary to investigate new techniques that may lead to enhanced perspicuity of the problems that DAT or other patients encounter in the course of their disease. 
Two distinctions have emerged that seem to be important to the understanding of the amnesic syndrome. Recently, new techniques based on paradigms from experimental psychology have been used to characterize the nature of the memory deficilt of diseases with different etlology. One distinction is that between episodic and semantic memory (Tulving, 1983). Semantic memory refers to general, organized knowledge, including the rules that govern the use of language and the meaning of words and concepts. It stands in contrast to episodic memory-the autoblographical memory for personal, unique events that are related to specific contexts.

Several studies have been done based on this theoretical dichotomy. Most of this research has involved patients with alcoholic Korsakoff's syndrome or other forms of amnesia. Generally, amnesic patients are believed to be unimpaired on tests that concern semantic memory but to suffer from a major deficit in episodic memory. In a case study, Tulving, Schacter, Mclachlan, and Moscovitch (1988) report on a patient with dense retrograde and anterograde amnesia. This patients has gross episodic memory deficits and virtually no semantic deficits. He can remember certain facts (i.e. semantic information) about his past but does not remember any of the circumstances of the major events in his life (i.e. episodic information). Given that relevant semantic knowledge is available "he is quite able to make the appropriate inferences from this Information, which may sometimes be quite puzzling in interpreting the nature of his disturbances. Another case report is presented by Wilson and Baddeley (1988). Their patient shows an impaired performance on a wide variety of episodic memory tasks but virtually no impairments on semantic tasks. However, in contrast to the patient of Tulving et al. (1988), this patient can recall autobiographical events; moreover, he even shows recollection of the details of the original experience. They argue that the existence of two separate memory systems is not at all clear. Theoretically, it is possible that semantic memory is an accumulation of many episodes (see also McClelland \& Rumelhart, 1985). An practically, it is often difficult to distinguish between what is episodic or semantic. For example, striking events and their circumstances may have been recollected often and thus repetition of these events might have resulted in their becoming part of semantic memory.

Although the evidence for a distinction between episodic and semantic memory is still a matter of discussion, it offers a very useful theoretical framework in the research of memory. Weingartner et al. (1982) argue that DAT patients show a deficit in semantic memory that is not found in other forms of amnesia. They propose that semantic structure and organization are preserved but that DAT patients are unable to access and utilize this semantic information. The problem of not being able to gain access to and utilize semantic information then accounts for the memory disturbances DAT patients exhibit on various tests of episodic memory.

A second distinction that seems to be important is that between implicit and explicit forms of memory testing (Schacter \& Graf, 1986b). In traditional memory testing subjects are well aware of the fact that the task they have to perform concerns memory. With these kinds of tasks subjects are explicitly instructed to remember and reproduce certain information (e.g. recall and recognition). In recent years, evidence has accumulated that amnesic patients show normall retention when memory is tested using procedures that do not involve conscious recall (implicit memory testing: e.g. priming, Cohen \& Squire, 1980; Jacoby \& Witherspoon, 1982; Shimamura \& Squire, 1984). Graf ef al. (1984) report that Korsakoff patients are unable to reproduce information when they are explicitly instructed to remember the presented information on an episodic memory task. However, when implicit instructions are given and attention is not directed at memory functioning, they are well able to remember that 
same information. Whether the same pattern holds true for DAT patients is not clear. Nebes et al. (1984) tested DAT patients on a semantic priming task. Both the experimental and the control group showed the priming effect, although it was rather small. They proposed that DAT patients may perform normally provided memory is tested under conditions that do not require effortful processing.

This phenomenon of preserved implicit memory and deficits in explicit memory capacity has been explained by a number of dichotomies; for example, automatic vs. controlled processing (Schneider \& Shiffrin 1977; Shiffrin \& Schneider, 1977), or procedural vs. declarative memory (Cohen \& Squire, 1980). Which of these dichotomies describes the results best is still a matter of dispute. The purpose of the present study is to investigate whether DAT patients perform normally when episodic memory is tested under implicit task instructions. A suitable task to test memory in an implicit way is the perceptual identification task (Jacoby, $1983 \mathrm{a}, \mathrm{b}$; Feustel et al., 1983). In this task words are presented for a very short duration. Subjects have to identify these words. The only instruction given to the subject is to read the word aloud. Although DAT patients show a number of deficits in language functioning, Cummings, Houllhan and $H$ ill (1986) found that reading words aloud is preserved until the very last stages of the disease.

The core of the perceptual identification task is that some of the words are repeated. It has been shown that a facilitation effect results due to previous exposure. The second time a word is presented, the probability of correct identification is increased. It is still not clear what kind of activation is responsible for the facilitation effect. Jacoby and Dallas (1981) attribute the facilitation effect to the activation of the episodic memory trace resulting from the first presentation (see also Salasoo et al., 1985). Support for this hypothesis comes from the work with normal subjects in which facilitation of pseudowords is studied (Cermak et al., 1985). They showed that when pseudowords are used as stimulus material (for which no semantic representation exists), normal subjects exhibit a normal facilitation effect. However, in the same experiment amnesic patients did not demonstrate a facilitation effect for pseudowords but did show facilitation for normal words. The results of the amnesic patients support an alternative hypothesis -namely, that facilitation depends upon the activation of preexisting representations in memory (Diamond \& Rozin, 1984; Graf et al., 1984). If memory deficits of DAT patients on episodic tests are only a result of not being able to access and utilize semantic information and implicit memory is preserved, a possible hypothesis would be that DAT patients will exhibit the same amount of facilitation as normal, age-matched controls. When tested on an explicit memory task, there will be a difference between the two groups. To test episodic memory with explicit task instructions, a forced choice recognition test was given after the perceptual identification task.

\section{EXPERIMENT 1}

In the first experiment, the performance of 11 patients suffering from probable Alzheimer's disease was compared to that of 11 age-and sex-matched controls. 


\section{METHOD}

\section{Experimental Subjects:}

DAT Patients. From a population of patients with memory problems from the Regional Hospital of Helmond/Deulne (The Netherlands), 11 patients were selected who met the criteria for primary degenerative dementia described in DSM III (Diagnostic and Statistical Manual of Mental Disorders, 1980), and NINCDS-ADRDA criteria for probable DAT (McKhan et al., 1984). Patients who came to their physician with memory complaints were first examined physically and neurologically at the hospital. This assessment consisted of examination of blood and urine, thorax photo, EEG, Hachinsky Scale, and Hamilton Depression Scale. If the symptoms could not be attributed to a specific cause, neuropsychological assessment procedure followed. This consisted of an anamnestic interview with the patient and with the spouse or the child(ren), the WAIS, the Trallmaking Test (version $A+B$ ), the Rey Complex Figure test (CFT), the Bender Gestalt Test, a test of attention (Attention Diagnostic Method), and the 15-Woorden Test (a Dutch standardized test; after learning and recalling a list of 15 unrelated words in five consecutive trials, subjects have to recall the items after a $30-\mathrm{min}$. dellay, followed by yes/no recognition). Only if no definite cause of the symptoms could be detected based on the results of the complete physical, neurological, and neuropsychological examination, were patients assigned to the group of probable DAT patients. This resulted in a group of nine women and two men varying in age between 54 and 86 (mean age $=74.7, \mathrm{SD}=9$ ); their mean education was 7.2 years. All patients were moderately to severely impaired: one patient with a GDS-score (the Global Deterioration Scale; Reisberg et all. 1982) between four and five, five patients with a GDS-score five, and five patients with a score of six. Four patients were neuropsychologically re-examined between six and twelve months later and proved to have deteriorated on all tests. One other patient had a cerebro-vascular accident and had developed an aphasia in the meantime, and thus comparable test results could not be obtained. Two patients had moved to a nursing home and were not longer testable according to their physician. The remaining four patients could not be traced.

The Control Group. The control group consisted of nine women and two men varying in age between 62 and 85 (mean age $=74.2, S D=7$ ); their mean education was 7.9 years. These subjects were paid for their participation. Screening of the control group consisted of an anamnestio interview, the 15-Woorden Test, the Rey Complex Figures Test, and the Trailmaking Test (version $\mathrm{A}+\mathrm{B}$ ). None of these subjects had complaints concerning their memory or any history of major neurological or psychiatric disease. The two eldest subjects ( 82 and 85 ) of the control group were slightly deteriorated on all tests compared to the standards of their age group.

\section{Matorial:}

Stimuli were presented via an Apple lle computer. Attached to it were a second (monochrome black/white) monitor and a button, with which subjects could start the presentation of a stimulus. Words were presented in lower-case letters. Character size was $5.7 \mathrm{~mm} \times 6.6 \mathrm{~mm}$. The perceptual identification task consisted of two parts: a training session and a test session. 
In the training session a list consisting of 50 words of intermediate frequency $(6 / 100,000<$ freq. < 90/100,000; Uit den Boogaart, 1975) was presented. During the test session two lists of words, each containing 42 high- and 42 low-frequency words (resp. treq $>90 / 100,000$ and freq. $<6 / 100,000)$ were presented. From each category 44 words were presented once, 14 twice, and 14 three times, resulting in 168 presentations. Repetitions were randomly dispersed across the list. The forced-choice recognition task consisted of 24 choice items of four words each. From each category of the stimulus material of the perceptual identification task (once, twice, three times presented $\mathrm{X}$ high and low frequency) four words were selected for use as target items. With each target item three other words were presented to serve as istractors. This was done for both lists of the perceptual identification test.

\section{Procedure:}

The training session was given to familiarize the subject with the procedure and to determine base-line performance in order to avoid floor or ceiling effects in the test session. All subjects had normal or corrected-to-normal vision. Each was asked to press the button, to look at the screen, and to read aloud the word presented on the monitor. First a trame consisting of lines was generated in the centre of the monitor. This frame stayed on the screen until the test was. completed. Stimuli were flashed within this frame for a very short duration. A mask consisting of a row of asterisks immediately followed the stimullus. This mask disappeared 1 sec after the subject had read the word. If the subject could not read the word or made an error, auditory feedback was given. Sitarting exposure was set at $200 \mathrm{msec}$. If an individual could not read any of the stimuli at $200 \mathrm{msec}$, the program was re-initialized, and starting exposure was increased by 140 msec. After presentation of a block of 10 words $_{n}$ stimulus duration was shortened by 40 msec. When the subject had read all five blocks of 10 words, the presentation duration was taken as base-line duration, which rendered a probability of correct identification that came closest to $40 \%$. This duration remained constant during the test session of the experiment. In this way base-line performance level was more or less equalized for all subjects. (An accurate $40 \%$ levell could not be reached because the reset cycle of the screen tekes 20 msec. This poses a problem for exact base-line determination of stimulus duration time, because of the individuall variation in visual capacity.) During the test session instructions and word presentation were the same as in the training session. After each test session of perceptual identification the (associated) forcedchoice recognition task was given. Subjects were instructed to read the words that appeared on the screen and to choose the word they had seen before in the perceptual identification task. If in doubt, they were encouraged to choose the word that seemed most probable. Four words were presented at a time (the target and three distractors). There was no restriction as to the exposure time. After a choice had been made, the answer was registered by the experimenter, and the next choice item appeared. 


\section{RESULTS}

Two subjects, one in the patient group and one in the control group, could not read the stimull at $200 \mathrm{msec}$. Their base-line presentation durations were 320 and $300 \mathrm{msec}$, respectively. Preliminary analysis revealed no difference between the results of the two lists of perceptual identification and recognition, so data were combined in the final analysis. The third presentations of words that were three times presented added no new information and were not further analyzed. Data of the first presentation and the second presentation of words that were presented twice and three times were combined. Data of the perceptual identification test and the recognition test were analyzed with ANOVA in a $2 \times 2 \times 2$, Group (DAT Ws controls), Frequency (high vs. low). Facilitation (first vs. second presentation of words) design, with repeated measures on the last two factors. In Table 1 the results of the perceptual identification task are represented.

Both groups show a higher percentage of correct identification the second time a word is presented, $F(1,20)=24.2, p<0.01$. On average, low-frequency words are less often correctly identified than high-frequency words, $F(1,20)=21.2, p<0.01$. However, the critical term group by facilitation is not significant $[F(1,20)=0.4]$, which means that both groups do not differ in the amount of facilitation in the perceptual identification task. The facilitation effects of the subject groups of high- and lowfrequency words are represented in Figure 1.

As it is possible that differences in the base-line presentation duration reflect qualitative differences in processing, an analysis of covariance was performed with stimulus exposure time as covariate. The results of this analysis are essentially the same as for the analysis of variance: The two groups do not differ in the amount of facilitation $[F(1,19)=0.2]$. The only difference is a disappearance of the frequency effect $\llbracket F(1,19)=2.0]$. The results for recognition memory are represented in Table 2 .

There exists a clear difference in recognition performance between DAT patients and controls, $F(1,20)=29.4, p<0.01$. Low-frequency words are more readily recognized than high-frequency words, $F(1,20)=5.4, p<0.05$. However, no clear facilitation effect is found for recognition memory $[F(1,20)=3.0]$. Analysis of covariance on the data of recognition memory again reveals a disappearance of the frequency effect $[F(1,19)=$ 0.81.

The base-line presentation duration was analyzed nonparametrically by a MannWhithey test for tied ranks. No difference was found between DAT patients and the control group (mean $=134.6 \mathrm{msec}, \mathrm{SEM}=21$, and mean $=117.3 \mathrm{msec}, \mathrm{SEM}=24$, respectively; $z=0.888$ ).

The results presented above confirm our hypothesis that, when using a perceptual identification test in which attention is directed to the reading of words and no conscious effort to remember items is involved. DAT patients do perform as well as normal elderly. However, when using a memory test for which subjects are explicitly instructed to search their memory for items previously presented, a significant difference arises between DAT patients and normal elderly subjects. The results of this experiment suggest that, when a minimal contribution of semantic memory is required and when task instruction is implicit, DAT patients can perform normally. Another, more unexpected, phenomenon can be observed in this study. The amount of facilitation of the control group is less than expected (see Jacoby, 1983b). However, we used a slightly different procedure than Jacoby, who presented words in a study list that were repeated in the perceptual identification task. One explanation is that the difference in procedure is responsible for the smaller facilitation effect in this study. 
Table 1: Mean Percentage of Correctly Identified Words as a Function of Frequency (HIgh- and Low-Frequency Words) and Repetition (First and Second Presentation; SEM between

Parentheses).

\begin{tabular}{l|llll} 
& \multicolumn{2}{|c}{ DAT } & \multicolumn{2}{c}{ Control } \\
& High & Low & High & Low \\
& & & & \\
\hline First presentation & $54.7(4)$ & $44.8(4)$ & $51.6(4)$ & $41.7(4)$ \\
Second Presentation & $63.1(4)$ & $50.7(6)$ & $58.8(3)$ & $52.9(4)$
\end{tabular}

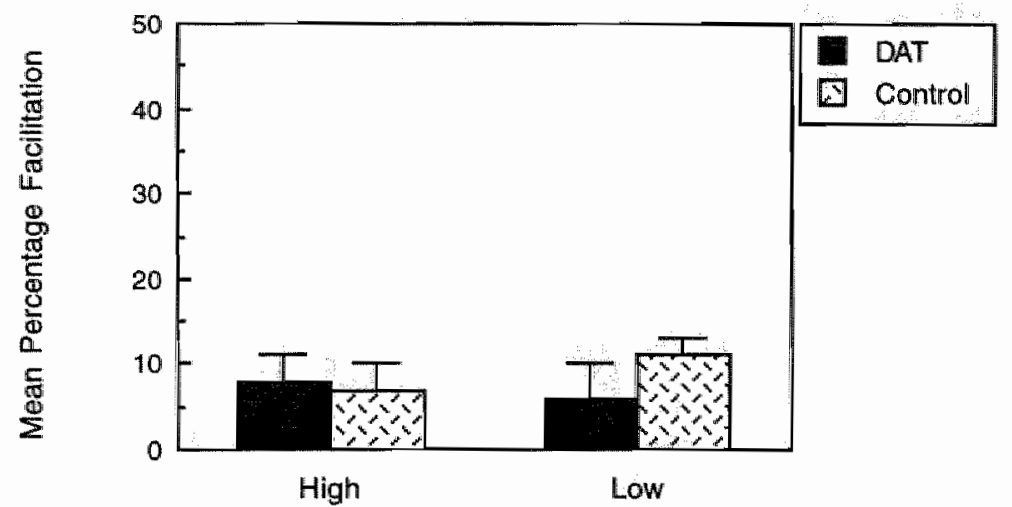

Figure 1: Facilitation Effects of DAT Patients and Normal Elderly.

Table 2: Mean Percentage of Correctly Recognized Target Items as Function of Frequency (High- and Low-Frequency Words) and Repetition (First and Second Presentation; SEM between Parentheses).

\begin{tabular}{l|cccc} 
& \multicolumn{2}{|c}{ DAT } & \multicolumn{2}{c}{ Control } \\
& High & Low & High & Low \\
\hline Once Presented & $28.4(5)$ & $38.6(6)$ & $46.6(5)$ & $63.5(5)$ \\
Twice Presented & $39.8(5)$ & $40.9(8)$ & $58.0(4)$ & $64.8(4)$
\end{tabular}

An alternative explanation is that the reduction of the amount of facilitation is agedependent. Therefore, a second experiment was done to explore the hypothesis that this automatic memory process of episodic memory diminishes as a result of ageing. 


\section{EXPERIMENT 2}

\section{Experimental Subjects:}

In the second experiment 11 normal elderly subjects and 11 young normal subjects were tested on perceptual identification and recognition. All subjects were pail for their participation. The elderly group consisted of four men and seven women, age varying between 62 and 85 (mean age $=72.8_{\text { }} \mathrm{SD}=7$ ); their mean education was 11.2 years. Performance of this elderly group was compared to that of a group of students, consisting of five men and six women, varying in age between 20 and 25 , with a mean of $23.3(S D=2)$; their mean education was 16.6 years. The base-line procedure was essentially the same as in Experiment 1 , with the exception that starting exposire time for the younger group was set at $100 \mathrm{msec}$. It was reduced by $20 \mathrm{msec}$ over each of the five successive blocks of 10 words. One individual in the elderly group could not read the stimull even at $340 \mathrm{msec}$. His base-line presentation duration was finally computed to be $700 \mathrm{msec}$. It is not clear why this subject needed so much time. The testing procedure was identical to that of the first experiment.

\section{RESULTS}

Data are analyzed in the same way as in Experiment 1. Results of the perceptual identification task are shown in Table 3, those of the recognition test in Table 4, Both groups show a significant facilitation effect of perceptual identification, $F(1,20)=158.8$, $p<0.01$. In this experiment the critical Group $X$ Facilltation interaction term is significant $F(1,20)=11.6, p<0.01$, which means that the groups differ in the amount of facilitation (See Figure 2). The facilitation effect for the elderly group is considerably less than that of the young group.

Percentage facilitation for the low-frequency words is higher than for the highfrequency words, resulting in a significant frequency effect, $F(1,20)=10.5, p<0.01$. As in the first experiment, an analysis af covariance was performed on the data with stimulus presentation duration as a covarlate. Agaln we find the same results as with the analysis of varlance; The young group exhibits a greater amount of facilitation, $F(1,19)=9.5, p<0.01$. High-frequency words are better identified than low-frequency words, $F(1,19)=8.2, p<0.01$. Words are more readily identified at second presentation than at first presentation, $F(1,19)=109.0, p<0.01$. The stimulus presentation duration was tested nonparametrically by a Mann-Whitney test for tied ranks. Elderly subjects needed longer exposure times than young subjects ( mean $=132$ msec, $S E M=58$, and mean $=27$ msec, $S E M=3$, respectively; $z=3.79, p<0.01$ ).

The young group recognized significantly more words than the older group, $F(1,20)$ $=16.2, p<0.01$. Also, a facilitation effect can be observed for recognition memory, $F(1,20)=12.0, p<0.01$. However, the interaction between group and facilitation is not significant $[F(1,20)=0.7]$, which means that both groups exhibited the same amount of facilitation on the recognition test. Recognition of the elderly group in this experiment is slightly higher than those in Experiment 1. This might be caused by a higher educational level. This higher educational level did not influence the perceptual identification task, as this task involves automatic processing (Hasher \& Zacks, 1979). Percentage facilitation on perceptual identification is approximately the same for the normal elderly group of Experiment 1 and of Experiment 2. 
Table 3: Mean Percentage of Correctly Identified Words as a Function of Frequency (High- and Low-Frequency Words) and Repetition (First and Second Presentation; SEM between parentheses).

\begin{tabular}{l|cccc} 
& \multicolumn{2}{|c}{ Young } & \multicolumn{2}{c}{ Old } \\
& High & Low & High & Low \\
& & & & \\
\hline First presentation & $43.9(5)$ & $37.7(6)$ & $50.5(4)$ & $40.1(4)$ \\
Second Presentation & $57.8(6)$ & $57.0(6)$ & $57.4(4)$ & $52.3(3)$
\end{tabular}

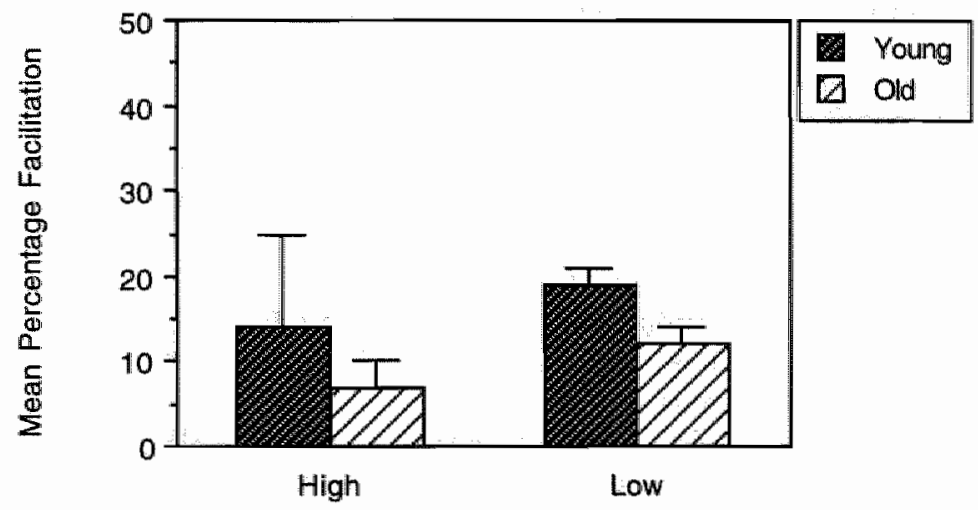

Figure 2: Facilitation Effects of the Young and the Old Adults.

Table 4: Mean Percentage of Correctly Recognized Target items as a Function of Frequency (High- and Low-Frequency Words) and Repetition (First and Second Presentation; SEM between Parentheses).

\begin{tabular}{l|cccc} 
& \multicolumn{2}{|c}{ Young } & \multicolumn{2}{c}{ Old } \\
& High & Low & Hight & Low \\
& & & & \\
\hline Once Presented & $84.1(5)$ & $83.4(4)$ & $59.1(6)$ & $53.4(6)$ \\
Twice Presented & $89.4(3)$ & $94.7(2)$ & $71.6(8)$ & $67.1(7)$
\end{tabular}

Analysis of covariance of the data of the recognition test reveals a difference between groups in recognition memory, $F(1,19)=10.5, p<0.01$. Also, a facilitalion effect can be observed for recognition memory, $F(1,19)=12.1, p<0.01$. 
The most striking result of the present study is that normal elderly and DAT patients are both impaired in their performance on the perceptual identification task. In other words, if episodic memory is tested implicitly, DAT patients were not impaired, compared to normal age-matched controls, but both the elderly groups showed impaired performance in comparison with young subjects. If memory is tested explicitly, as with the forced-choice recognition task, all elderly groups performed worse than young subjects, although DAT patients did so to a greater extent.

Our findings closely resemble those of Moscovitch (1982). He first tested implicit memory by letting young, old, and memory-disturbed subjects read sentences and timing their reading speed. After several delays old and new sentences were presented. Again the reading speed was registered, and the subjects were asked whether or not they had seen the sentence before. He found that the DAT patients read old sentences that were presented twice faster than sentences that they had seen only once. Sentences that had never been presented were read most slowly. This pattern was also found in the normal elderly. Both groups performed worse than the young group. Recognition memory was worst for the memory-disturbed subjects and best for the young adults. Interestingly, in a further experiment he tested the same subjects on repetition priming in a lexical decision task with words and nonwords. In contrast to the findings of the previous experiment, he found that the normal elderly participants exhibited unimpaired priming, as did the DAT group.

Sometimes preserved implicit memory in DAT, as in amnesia, is offered as an explanation for the pattern of memory disturbances these patients exhibit (Morris \& Kopelman, 1986). For example, Gabrieli (1989) reports preserved perceptual priming in a perceptulal identification task of DAT patients. Implicit memory in DAT, however, is not always found to be intact. For example, the findings of Salmon et al. (1988) contradict this hypothesis. They found an impaired performance of the DAT group of lexical priming on a word-stem completion task, in contrast to a preserved performance of Korsakoff patients and patients with Huntington's disease.

Several authors stress the fact that there may be parallels between the memory disturbances that accompany DAT and normal ageing (Corkin, 1982; Moscovitch 1982 ; Moscovitch et al., 1986). On many tests of episodic memory DAT patients as well as normal elderly do not perform as well as young subjects. A possible explanation for the poor performance on episodic tasks is sometimes sought in disturbances in semantic processing. For example, Weingartner et al. (1983) suggest that the deficit of DAT patients may lie in a lack of access to semantic structures, so that semantic information necessary for good performance on eplsodic tests does not become available. Rabbit (1984) offers a similar explanation for the memory impairments of the elderly: Elderly subjects are assumed to suffer from a semantic encoding deficit. Impairments on episodic memory tasks are attributable to the sensitivity of those tasks for the level of semantic processing (Burke et al., 1987). However, Jacoby and Dallas (1981) have shown that semantic encoding of stimuli or depth-of-processing manipulations do not affect facilitation, in contrast to recoginition memory.

Our results suggest that ageing affects automatic processing in episodic memory, but that DAT does not further aggravate the process of decline. This is contradictory to the notion that processes that require little conscious attention are less likely to degrade with age (Hasher \& Zacks, 1979). This suggestion, however, is in accordance with two studies (by Lehman \& Mellinger, and by Kausler et al.) reported by Reder, Wible, and Martin (1986), and with the results of Howard et al. (1986). The latter 
tested young and old adults on a semantic priming task. They used three different SOAs. The old group showed considerably less priming only at the short SOA which is presumed to mirror automatic processing. They interpreted their results in terms of a slowing in mental processing of the elderly. This explanation cannot be applled to the present findings, because presentation duration was individually assessed. The issue of preserved or impaired automatic processing is not yet settled, as different results have been reported (Craik \& Byrd, 1982; Light et al., 1986). Part of the confusion seems to be due to differing uses of such concepts as automatic and controlled processing. The differential effects of ageing and DAT on perceptual identification and recognition memory might be explained by assuming that recognition memory is much more sensitive to effort and strategies (controlled processing). Older "healthy subjects presumably benefit more from controlled processing than does the DAT group. It is possible that people may actively select among strategies to mitigate specific decrements that accompany old age and as such (partly) compensate for changes that occur with advancing age (Rabbit, 1982).

Some additional remarks concerning the perceptual identification task are in order. A first remark concerns the base-line procedure. The determination of the base-line presentation duration was rather crude, probably due to, as mentioned earlier, a large variation in visual capacity, especially in the elderly groups. Although variations in visual capacity can influence initial identification, it cannot be responsible for a diminished facilitation effect of the elderly groups, because this is a constant factor at the first and the second presentation. Analysis of covariance with stimulus exposure time as a covariate also reveals no differences in the observed effects on facilitation. However, the refresh-cycle of the monitor takes $20 \mathrm{msec}$, and this causes a problem that is especially obvious in the young group. Some subjects could read the words too well (rendering a ceiling effect) at $40 \mathrm{msec}$, but they could not read the stimuli at all at 20 msec (rendering floor effects). It would be appropriate to refine the procedure by, for example, more precise screen-control. Another point is that the range of the facilitation effect was rather small. It is imaginable that in this case the facilitation effect reaches an asymptote, which may be why additional changes as a result of DAT go unnoticed. Increasing the range of facilitation -by giving visual instead of auditory feed-back, for example- might reveal a difference between normal ageing and DAT.

The results of the present study might be summarized as follows: DAT patients and normal elderly exhibit a similar amount of facilitation on a perceptual identification task, but both are impaired compared to young subjects, indicating a loss of automaticity as a result of ageing. However, recognition memory of the elderly is not as poor as in DAT patients. A tentative explanation might be that the normally aged compensate for their loss of automaticity (for example, by relying more on controlled processing and choosing different strategies in recognition judgements), but that DAT patlents show an incapacity to compensate, and this is superimposed upon a loss of automaticity. 


\subsection{NO DIFFERENCE IN EPISODIC MEMORY PERFORMANCE BETWEEN DAT PATIENTS, DEPRESSED PATIENTS, AND NORMAL ELDERLY (EXPERIMENT 3)}

\section{INTRODUCTION}

In a previous experiment we tested the performance of moderately-to-severely impaired DAT patients and normal elderly on perceptual identification and recognition memory (Abbenhuis, Raaijmakers, Raaijmakers \& van Woerden, 1990, Exp. 1). Parallel to that study we investigated the performance of mildly impaired DAT patients, depressed patients and control patients on the same tests.

One of the major problems in the diagnosis of dementia of the Alzheimer type (DAT) is differentiating the disturbances that accompany DAT from those that are attributable to depression. DAT patients, especially in the very early course of the disease, may often deny problems and early intellectual changes may go unnoticed. The patient, however, may be very well aware of cognitive decline. As a result depressed mood, anxiety, loss of interest, decreased spontaneity, somatic complaints and irritability may lead to the impression that the DAT patient is suffering from an affective disorder. On the other hand, a depressed patient may utter complaints of intellectual deterioration and memory impairments. Cognitive decline in terms of psychomotor slowing and memory loss is often obvious in the early stages of DAT, but these characteristics may be very simillar to the impairments of elderly depressed patients (Hart et al., 1987). The difficulty of differentiating dementia from depression may be illustrated by the percentage of incorrect diagnosis of thoroughly evaluated patients with an initial diagnosis of dementia who, at follow-up, are revealed to be suffering from depression. Percentage of misdiagnosis may range from $5-15 \%$ (Feinberg \& Goodman, 1984) up to $60 \%$ (Gurland \& Toner, 1983). Although there are parallels in the symptomatology of depression and DAT, the latter is characterized by irreversibility, a progressive decline, and resulting from degenerative brain disease. This stands in contrast with the lack of progression, reversibility, and a functional causation of pseudodementia (Mahendra, 1985).

Depressed and DAT patlents may exhibit equal test performances in many traditional tests of memory. For example, La Rue, D'Ella, Clark, Spar and Jarvik (1986) compared depressed and demented patients on three widely used tests of memory. Although one of these test appeared to be sensitive to varying degrees of memory disturbance, they concluded that it was less diagnostically specific as one might wish. Also, Hart, Kwentus, Taylor, and Harkins (1987) found similar performances of their DAT group and their group of depressives on several traditional tasks.

In recent years, several studies have been done to evaluate the specific nature of the memory deficits of DAT and depression. These studies are based upon paradigms originating from experimental psychology which allow us to study mare closely the distinctive characteristics of the memory deficits accompanying DAT and depression. An important distinction is that between automatic and controlled, or effortful processing (automatic vs. controlled processing; Shiffrin \& Schneider, 1977). Automatic processing is not limited by the processing capacity of working memory because it is parallel in nature, nor is it influenced by strategles, practice, or motivational aspects. Controlled processing, on the other hand, is under conscious control, relles on 
strategies, and is serial in nature. Thus, it is limited by the processing capacity of working memory. Mostly, the impaired performance of depressed elderly is explained in terms of reduced effortful or controlled processing. The more effortful processing is required in a task, the greater the deficits depressed subjects exhibit (Ellis et al., 1984; Hart, Kwentus, Hamer, \& Taylor, 1987; but see OHara et al.; 1986). This impaired performance on memory tasks that require effortful processing is mainly attributed to motivational aspects, to the allocation of attentional resources, or to differences in the use or initiation of strategies (Hertel \& Hardin, 1990). For example, Roy Byrne, Weingartner, Bierer, Thompson and Post (1986) found greater impairments of depressed patients on tasks that require effort, particularly sustained effort. This followed from the observation that depressives were more impaired on tests of free recall than on tests of recognition, or on tests of free recall as opposed to tests of cued recall. They assumed that cued recall and recognition require less controlled processing than free recall.

However, as the disturbances in DAT tend to stretch to many domains of cognitive functioning, some assume that not only controlled processing but also the more basic aspects of memory (like automatic processing) are affected in DAT (Watts, Morris Macleod, 1987; Sallmon et al., 1988). However, the evidence for impaired automatic processing in DAT is not very clear-cut. Some authors report preserved automatic processing in DAT. For example, Nebes et al. (1984) demonstrated preserved semantic priming in DAT. Similarly, Moscovitch (1982) found normal repetition priming in DAT, as opposed to severe impairments in recognition memory. In our previous study (Abbenhuis et al., 1990), we also found preserved automatic processing in moderately to severely impaired DAT patients compared to normal, age-matched controls.

We will present the results of a study with mildly impaired DAT patients, depressed patients and patients who reported problems in remembering, but whose memory complaints could not be objectified by neuropsychological assessment. We selected a group of patients of varying etiology as control subjects, because some authors caution against the use of normal or exceptionally high-functioning control subjects (Johnson \& Magaro, 1987; La Rue et al., 1986). Any kind of pathology, be it DAT or depression, or diseases of a different etiology, might be the only important factor which determines the extent of cognlitive impairment, particularly impairment on tasks which involive effortful processing (Cohen, Weingartner, Smallberg, Pickar \& Murphy, 1982). As in our previous study we used two tests: one in which memory is implicitly tested (repetition priming; Jacoby, 1983 a,b; Feustel, Shiffrin \& Salasoo, 1983), as well as a test which explicitly assesses memory (recognition memory task). Taking the results of our earlier study into account we expect no differences between the performances of the three patient groups on perceptual identification.

\section{METHOD}

\section{Experimental Subjects:}

A total of 36 subjects from a population of patients from the Regional Hospital in Helmond (the Netherlands) was selected for the experiment. All patients underwent an exiensive physical, neurological and neuropsychological examination. They were assigned to the separate experimental groups based on the results of the total assessment. In general, the physical and neurological assessment included examination of blood and urine, thorax photo, EEG and, if 
indicated, CT-scan, the Hamilton Depression Scale and the Hachinsky Scale. Neuropsychological examination consisted of an anamnestic interview with the patient and with the spouse or the child(ren), the WAIS, the Trailmaking Test, the Rey Complex Figure Test, the Bender, a test of attention (the Attention Diagnostic Method), and the 15-Woorden Test (a Dutch version of the Rey Auditory Verbal Learning Test: see Lezak; 1983). All subjects had normal, or corrected-tonomal vision. The diagnosis is based upon the judgements of both a neurologist and a neuropsychologist. Assignment criteria will be delineated within the description of each group.

DAT Patients. These subjects received the diagnosis of probable DAT based on the criteria of the NINCDS-ADRDA (McKahn et al., 1984). Selection criteria included a modified Hachinsky score $<5$, a score $<14$ on the Hamilton depression scale, a modified Crichton score between 14 and 30, and GDS-levels of 4 (the Global Deterioration Scale; Reisberg et al., 1982). The subjects were all community-dwelling. All patients had memory complaints, which were confirmed in neuropsychological assessment, although the impairments were rather mild. Also, mild deficits in other domains of cognitive functioning were verified. Only if no definite cause of the symptoms could be detected based on the results of the complete physical, neurological, and neuropsychological examination, were patients assigned to the group of probable DAT patients. This resulted in a group of six women and six men varying in age between 52 and 79 (mean age = $64.4 n S D=8.4)$; their mean education was 10.8 years ( $S D=4.2)$. Eight patients were neuropsychologically re-examined between 2.5 and 3 years later. These patients revealed a mild to moderate decrease in performance on all tests. Three patlents appeared to have deteriorated more severely, and were no longer testable. One patient had died.

Depressed Patients. This group consisted of three men and nine women with ages varying between 39 and 8.4 years (mean age $=65.8, \mathrm{SD}=13.3$ ); their mean education was 8.6 years ( $\mathrm{SD}$ $=3.0$ ). All subjects suffered from major depression according to the criteria described in the DSM III (Diagnostic and Statistical Manual of Mental Disorders, 1980), and were known for prior depressive episodes. All patients were hospitalized until the depressive symptomatology was diminished to the extent that it did not interfere with daily living routines. Four of these subjects had, among others, specific complaints concerning their memory. These complaints were braced by neuropsychological testing. Also, six other patients proved to be more or less deteriorated on standard tests of memory. However, none of these patients were very motivated, and they were inclined to give up very easily. A reaction that was often heard trom these patients was: "I can't do this anymore". Four patients were neuropsychologically re-examined between 2.5 and 3 years later. Two patients still performed on the same level, and two patients had improved. The remaining eight patients refused to cooperate.

The Control Group. The control group consisted of nine men and three women with ages varying between 42 and 75 years (mean age $=61.4, \mathrm{SD}=10.5$ ); their mean education was 9.8 years (SD $=1.9)$. All subjects complained of memory fallure, but this could not be confirmed by neuropsychological assessment. Nine of them were referred to the hospital for examination by theili physician, because of excessive anxiety to become demented. One patient had suffered from a 
cerebral infarct, one patient was suspected to be suffering from TIA's, and one patient underwent a lobotomy of the left superior frontal lobe. Assignment criterion was subjective memory complaints, which could not be objectified by neurologic nor neuropsychological testing.

\section{Material and Procedure:}

Material and procedure were the same as in experiment 1 and experiment 2 in our previous study.

\section{RESULTS}

The data of the perceptual identification task and the recognition task were analyzed in the same way as in Experiment 1 and Experiment 2. In Table 5 the results of the identification responses of the perceptual identification task are presented, in figure 3 the facilitation effects are shown.

There is no difference between groups in the percentage correct identification of words the first time they are presented [resp. High-frequency words: $F(2,33)=0.4$; Low-frequency words $[F(2,33)=0.6]$. All groups show a higher percentage of correct identification the second time a word is presented, $F(1,33)=87.3, p<0.01$. On average, low-frequency words are less often correctly identified than high-frequency words, $F(1,33)=17.7, p<0.01$. However, the critical term group by facilitation is not significant $[F(2,33)=1.1]$, which means that the three groups do not differ in the amount of facilitation in the perceptual identification task.

Post-hoc Duncan analyses of the facilitation effects, also reveals no differences between groups. Stimulus presentation duration was analyzed with a Kruskal-Wallis one-way analysis of variance by ranks, corrected for ties: There is a difference in stimulus presentation duration between the groups: $H(d f=2)=18.6, p<0.01$. (DAT: mean = 83.3 msec $($ SEM $=23.2) ;$ DEP: mean $=125.0$ msec $($ SEM $=36.3) ;$ CON: mean $=$ $75.0 \mathrm{msec}(\mathrm{SEM}=10.8)$ ). As in our previous experiments (Abbenhuis et al., 1990) an analysis of covariance was done with stimulus presentation duration as a covariate. The results of this analysis are essentially the same as for the analysis of variance, which means that the difference in stimulus presentation duration has no influence upon the similar facilitation effects of the three groups.

The results for recognition memory are presented in Table 6 . There is only a marginal difference in recognition performance of the three groups, $F(2,33)=3.0, p=0.06$. Post-hoc Duncan analyses only reveals a difference on recognition of low-frequency words after repeated presentation between DAT and depressed patients on the one hand and control patients on the other hand. Words are more readlly recognized after repeated presentation than after a single presentation, $F(1,33)=9.0, P<0.01$. There is also a marginal interaction between frequency and number of presentations, $F(1,33)$ $=3.9, p=0.06$.

Analysis of covariance with stimulus presentation duration as a covarlate results in the disappearance of the marginal differences between the three groups. 
Tabile 5: Mean Percentage of Correctly ldentified Words as a Function of Frequency (High- and Low-Frequency. Words) and Flepetition (First and Second Presentation; SEM between parentheses).

\begin{tabular}{l|cccccc} 
& \multicolumn{2}{|c}{ DAT } & \multicolumn{2}{c}{ Depressed } & \multicolumn{2}{c}{ Control } \\
& High & Low & High & Low & High & Low \\
\hline First Presentation & $53.6(3)$ & $44.4(5)$ & $56.3(4)$ & $48.4(4)$ & $51.8(3)$ & $42.6(3)$ \\
Second Presentation & $62.4(2)$ & $56.0(4)$ & $67.3(3)$ & $61.9(5)$ & $64.7(4)$ & $59.8(4)$
\end{tabular}

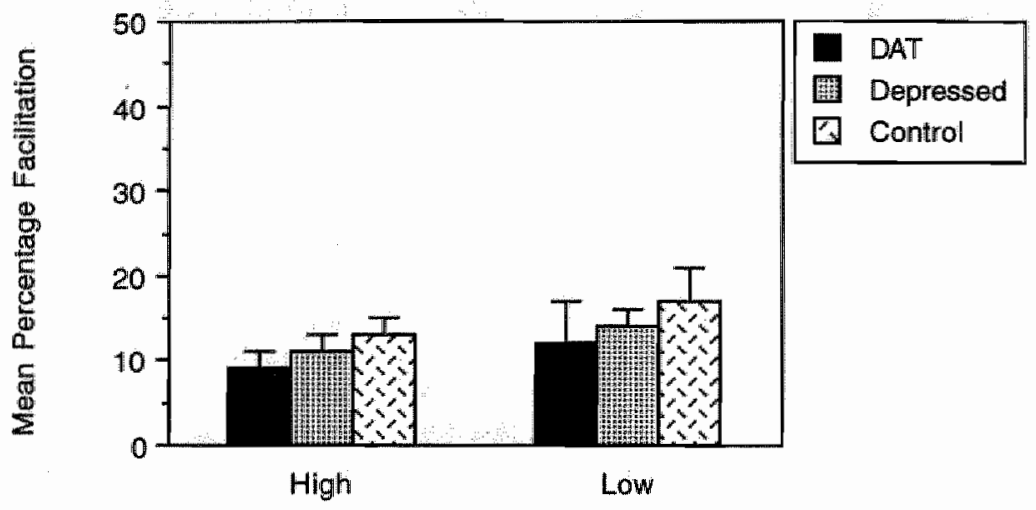

Figure 3: Mean Percentages Facilitation of the DAT Patients, the Depressed Patients and the Control Group as a Function of Word-Frequency.

Table 6: Mean Percentage of Correctly Recognized Target Items as a Function of Frequency (High- and Low-Frequency Words) and Repetition (First and Second Presentation; SEM between Parentheses).

\begin{tabular}{l|cccccc} 
& \multicolumn{2}{|c}{ DAT } & \multicolumn{2}{c}{ Depressed } & \multicolumn{2}{c}{ Control } \\
& High & Low & High & Low & High & Low \\
\hline Once Presented & $43.8(5)$ & $55.2(6)$ & $50.0(5)$ & $55.2(7)$ & $56.3(7)$ & $61.5(5)$ \\
Twice Presented & $70.8(4)$ & $54.2(5)$ & $60.4(3)$ & $51.0(7)$ & $65.6(6)$ & $72.9(6)$
\end{tabular}




\section{DISCUSSION}

The results of the present study can be summarized as follows:. There is no difference in the automatic retrieval of an episode between DAT patients, depressed patients and control patients. Concerming recognition memony, only a marginal difference can be seen between the three groups, attributable to an impaired recognition of low-frequency words of DAT and depressed patients compared to the control group. The presentation duration differs between groups with the depressed patients requiring the longest time to correctly identify about $40 \%$ of the words.

These results seem to support our hypothesis that automatic processing is presenved in DAT, as well as in depression. That is, if we assume that the control group we tested shows an unimpaired performance. Alternatively, it is possible that all three groups are impaired in automatic memory performance. This is, however, not probable as the facilitation effect of the control groups of the previous experiments (Abbenhulis et al., 1990) are even somewhat smaller than that of the control group of the present experiment.

The results of the DAT patients on the perceptual identification task are similar to those of our previous study (Abbenhuis et al., 1990; Exp: 1): DAT patients show a similar amount of facilitation as the control group. Also, the amount of facilitation of the present experiment is comparable to that of the previous one (mean percentage: 7.2 vs. 10.2). These results are in contrast with the findings of Salmon et al. (1988) and Shimamura et al. (1987). They tested DAT patients on a word-completion task and found an impairment of DAT patients compared to age-matched controls. The former authors also found no semantic priming effects of their DAT patients. Partridge, Knight and Feehan (1990), however, found unimpaired word-completion performance in their DAT patients. They attribute the discrepancy of their results and the results of Salmon et al. (1988) and Shimamura et al. (1987) to the nature of the orlenting task, and the amount of attentional processing that is necessary. However, in recent publications it is suggested that various implicit memory measures, like wordcompletion and perceptual identification, may differ in their underlying processes (Hunt \& Toth, 1990; Perruchet \& Baveux, 1989), so our results on perceptual identification and the results of the abovementioned studies may not be directly comparable.

Grafman et al. (1990) compared DAT patients with patients suffering from Progressive Supranuclear Palsy (PSP) on a perceptual identification task. They found preserved perceptual priming in the DAT group. They suggest that the longer exposure times of the DAT group may have confounded the finding of preserved perceptual priming. However, we did not find an effect of exposure duration on the facilltation effect. In the same article Grafman et al. (1990) report the results of an experiment in which they compared DAT patients, depressed patients and normal controls on another perceptual priming task in which they used the Gollin Figures as stimulus material. They presented the figures on two consecutive days and registered the time needed to "dentify each object. DAT patients as well as depressed patients showed savings from one day to the other, although DAT patients were much slower. On an explicit recognition memory task, both DAT patients and depressed patlents performed worse than controls. Our findings are in accordanice with these results both for the priming effect and the results on the recognition memory task.

Most studies which directly compare DAT patients, depressed patients and normal elderly use standard clinical memory tests. The problem with these kinds of tasks is that the underlying mechanisms of good or impaired performance are largelly unknown, and it is not surprising that conflicting results are found. For example, Kopelman 
(1986a) compared DAT, Korsakoff, depressed patients, and normal controls on several verbal learning tasks. The depressed differed from the DAT patients on all measures but also from the control subjects, except on Digit Span. Hart et al. (1987c) used an incidental memory task based on the Digit Symbol subtest of the WAIS. DAT patients performed worse than depressed and controls both on the recall of symbol pairs and the total number of recalled symbols. Depressed patients; on the other hand, only performed worse than controls on the more difficult task: the recall of symbol pairs. In another study, Hart et al. (1987b) found equal performance of depressed patients and control subjects on the recognition of previously presented linedrawings of common objects. Both groups performed better than DAT patients. Hasher and Zacks (1979) used a similar recognition procedure as we did and found no impairment of their depressed group. However, their subjects were considerable younger than ours. It is quite possible that the higher age of our depressed subjects is responsible for the discrepancy between their study and ours.

This leaves us to some final remarks on the relation between the results on perceptuall identification and recognition of the previous and the present study. The assumption that ageing does have an effect on automatic processing, although not directly addressed in the present study, might be supported by the observation that our present (younger) control group performs better than the (older) control groups of the earlier experiments. In fact, they exhibit almost the same percentage facilitation as the young group in experiment 2 . This could serve as an indication that the effects of ageing on automatic processing only become noticeable at the higher ages. This issue will be further addressed in the next chapter.

The DAT group of the present experiment, although they are younger, perform at a similar level as the older and more severely impaired group of the previous experiment. This could mean that the effects of ageing become evident at an earlier age in DAT patients. However, the lack of a further deterioration of tacilitation in the more severe group could also mean that the observed amount of facilitation is the result of a flooreffect. In other words, there may be minimal amount of facilitation. The DAT groups, as well as the older control groups may exhibit this minimal amount of facilitation, so that no further deterioration can be manifested as a consequence of DAT.

For recognition memory a different pattern can be seen: Mildly impaired DAT patients recognize more words than moderately-to-severely impaired DAT patients. But control subjects under the age of 70 recognize a similar percentage of items as subjects over the age of 70 do. 


\section{Chapter 3}

\section{Perceptual identification with visual feedback}

"He was wandering inside himself, and he was lost. [..] His life no longer seemed to dwell in the present .

Paul Auster: The Invention of Solitude.

\subsection{INTRODUCTION}

In Chapter 2 we first examined the hypothesis that DAT patients, whose disturbances stretch to various domains of cognitive functioning, would suffer from impairments in automatic processing, as well as from controlled processing deflcits (Cohen et al., 1982; Newman et al., 1984; Roy-Byrne et al., 1986). Secondly, we examined the hypothesis that depressed patients exhibit no impairments in automatic processing, in contrast to the impairments which are presumed to occur in DAT patients, an assumption which could be of value in the differential diagnosis between DAT and depression (Hasher \& Zacks, 1979; Silberman et al., 1983). And thirdly, we tested the hypothesis that automatic processing is preserved in normal ageing (Hasher \& Zacks, 1979; Light \& Singh, 1987).

We did not, however, find support for these assumptions: With respect to the first hypothesis, mildly impaired, and moderately-to-severely impaired DAT patients did not differ from age-matched control groups on the automatic retrieval of words when tested on a perceptual identification task. Moreover, the amount of facilitation of the DAT patients in experiment 1 and experiment 3 is approximately the same. Given that we did not find a difference between DAT patients and control subjects, it is not surprising that we could not observe any difference between DAT and depressed patients. Thus, we could not find any evidence in support of the hypothesis that depressed subjects perform better than DAT patients on the perceptual identification task. 
With respect to the effect of ageing, however, a different pattern was seen. In experiment 2 we found an age-related deterioration of the facilitation effect. However. the elderly control group of experiment 3 , which was more than ten years younger than the elderly control groups of experiment 1 and 2 , seemed to perform almost as good as the young group of experiment 2 . Although the results of the various experiments in Chapter 2 are not directly comparable, they might serve as an indication that, first, ageing negatively affects automatic processing, but only at the higher ages. And second, that progression of DAT does not further exacerbate this impaiment.

A possible explanation for the failure to find a difference between the DAT and the depressed patients could be that the amount of facilltation of the two groups reflects a floor-effect. In order to avold possible floor-effects we changed the procedure to increase the range of the facilltation effect. A feasible reason why we found an agerelated deficit is that the size of the letters was small. This may impose particular problems especially in all the elderly groups. Furthermore, as has been proposed in the discussion of experiment 1 , it was difficult to assess the base-line presentation duration because of the limitation of the screen refresh-cycle of the monitor we used.

Therefore, we changed the experimental procedure as follows: We developed a display which permitted improved timing resolution. The screen we developed was composed of LED-displays. Timing resolution is much more accurate as presentation durations can be increased by intervals of 1 millisecond. (however, the memory capacity of the Apple $l l e$ computer presented an additional restriction, so stimulus duration could only be actually increased by 5 milliseconds). An additional advantage is that the standard letter-size of this screen is larger than that of the one we used before. In addition to the modification of the apparatus we changed the mode of feedback: Instead of giving auditory feedback in case of an incorrect identification, visual feedback was given.

To our knowledge, there is only one study which examined the effect of feedback upon perceptual Identification performance. Jacoby and Dallas (1982, exp. 2a) investigated whether task difficulty in the encoding stage would differentially affect perceptual identification performance and recognition memory. They compared two encoding conditions: Solving an anagram of a word or simply reading the word. If the anagram was not solved the correct solution was told to the subject by the experimenter. The subsequent facilitation effect in perceptual identification was $12 \%$, in the next experiment (Jacoby \& Dallas, 1982, exp. 2b) the procedure was analogous, except that they provided visual feedback if the anagram was not solved (i.e. the correct solution was visually presented): Consequently, the observed facilitation effect was increased to $21 \%$, but the overall pattern of results was the same as in their previous experiment. These results are in accordance with the assumption of Hasher and Zacks (1979) who state that automatic processing should not be ameliorated by feedback about earlier performance. Therefore, we expected to find an increase in the range of facilitation, but not in the pattern of facilitation. That is, elderly subjects should show less facilltation than younger subjects as was the case in our previous experiments presented in Chapter 2 .

In paragraph 3.2 we will present the data of an experiment in which the performance of mildly-to-moderately impaired DAT patients was compared to that of a group of depressed patients and a group of normal elderly subjects. If we failed to find a difference in the amount of facilitation between the DAT patients on the one hand and the depressed patients and normal controls on the other hand (Chapter 2, experiment 3) because of a possible floor-effect, we expected to find a difference between the groups on the perceptual identification task with visual feedback in which range of 
facilitation was increased.

In paragraph 3.3 we will present the results of an experiment in which we further explored our hypothesis that a deficit in automatic processing develops with increasing age. Davis et al. (1990, exp. 2) tested seven different age groups on word-stem completion. Up until 70 years they found no evidence for impaired pertormance of the elderly. However, after the age of seventy their subjects performed worse than the younger groups. Similarly, Light and Singh (1987) tested young and older adults on both word-stem completion test (exp. 1 and 2) as well as on perceptual identification (exp. 3). Mean age of their elderly groups was comparable to that of our subjects in experiment 3. They found no impairment in their elderly groups on either of these implicit memory measures. The former authors attribute the difference between the two studies to the age of the eldest group in the latter study.

We were interested to find out at which age the impairment in the facilitation effect we observed in experiment 2 would arise. Therefore, we tested six age groups, varying in age between 20 and 80 . In accordance to the results of the study of Davis et al. (1990), we expected to find attenuated facilitation, but only so in the eldest groups. After finding a discrepancy between the task with visual and that with auditory feedback (1.e. finding an age-related impairment on the task with auditory feedback, but not on the task with visual feedback) we performed an experiment in which three age groups were compared on perceptual identification and recognition performance of words and nonwords.

As has been stated in Chapter 1, there is much discussion about whether the facilitation effect is based upon episodic or semantic processing or both. Support for the episodic hypothesis comes from studies that use pseudowords as stimulus material. There is no semantic representation of these words, and thus facilitation of pseudowords must be attributed to episodic memory processing. Feustel et al. (1983) found a facilitation effect for words, as well as for pseudowords in normal young subjects. However, Cermak et al. (1985, Exp. 2) found a dissociation between the perceptual priming of words and pseudowords in amnesic patients. In contrast to the normal priming effect they found for words, their amnesic patients exhibited impaired priming for pseudowords. They concluded that for priming to occur in amnesiacs there must be a semantic representation. Some authors suggest that there may be parallels between the disturbances that accompany ageing and the impairments which result from the amnesic syndrome (Corkin, 1982; Moscovitch, 1982; Moscovitch et al., 1986). To investigate whether similar results could be obtained In relation to ageing, we tested elderly subjects on implicit memory for words and nonwords. The results of this experiment will be presented in paragraph 3.4. Our expectations were that if the impairment of elderly people on perceptual identification with auditory feedback is only due to a deficit in processing episodic information an age difference should arise in the facilitation effect of nonwords for which no semantic representation exists. 


\subsection{A COMPARISON OF DAT PATIENTS, DEPRESSED PATIENTS, AND NORMAL ELDERLY ON A PERCEPTUAL IDENTIFICATION TASK WITH WORDS (EXPERIMENT 4)}

\section{METHOD}

\section{Experimental Subjects:}

The patient groups were selected on the same criteria as described in experiment 3.

DAT Patients. This group consisted of eight men and six women, varying in age between 56 and 86 (mean age: $72.9 ; S D=7.5)$. Their mean education 9.2 years $(S D=2.1)$. Seven patients were re-examined between 1 and 1.5 years later. Four of them revealed to be deteriorated on all neuropsychological tests, and three performed on essentially the same level. Four patients were not remexamined because the time between the initial and the repeated experiment was less than one year. of the remaining three patients one was still living at home. His spouse reported that he was too severely deteriorated to be tested again. The other two had been admitted to a nursing home.

Depressed Patients. This group consisted of three men and seven women, varying in age between 54 and 78 (mean age $=67.9 ; \mathrm{SD}=8.3$ ). Their mean education was 8.0 years $(\mathrm{SD}=$ 2.9). Four patients were re-examined between 1 and 1.5 years later. Three patients had improved on all neuropsychological lests, and one patient pertormed on essentially the same level. The remaining six patients refused to cooperate.

The Control Group. The control group consisted of subjects who were recruited by advertizing in a local newspaper. Prior to the experiment a checklist was given to assure no subjects had a history of present or prior alcohol abuse, neurological or psychiatric disease. All subjects were paid for their participation. The control group consisted of seven men and seven woman with ages varying between 64 and 77 (mean age $=70.0 ; \mathrm{SD}=5.4$ ). Their mean education was 9.6 years $(\mathrm{SD}=3.5)$.

\section{Material:}

Stimuli were presented via an Apple lle-computer. Attached to it was a screen composed of a series of 10 matrix-displays (SIEMENS Dot Matrix Intelligent Displays/DLG 7137, green). Each display ( 12.9 by $17.3 \mathrm{~mm}$ ) consisted of a matrix of $5 \times 7$ LEDs. Also connected to the computer were a button with which the presentation of a stimulus could be initiated, and a voice-key with which the onset of a response could be registered. Words varied in length between three and six letters and were presented in upper-case letters.

The perceptual identification task consisted of two parts: a training session and the test session. In the training session a list consisting of 50 words of intermediate frequency $\left(6 / 100.000<\right.$ freq $^{2}<90 / 100.000$; Uit den Boogaart $\left.{ }_{\mathrm{n}} 1975\right)$ was presented. During the test 
session a list of 100 words was presented. The test list contained 30 high and 30 low frequency words (resp. freq. $>90 / 100.000$ and freq. $<6 / 100.000$ ). Ten words of each frequency served as fillers and were presented only once. Twenty words of each frequency were presented twice. This resulted in a list of 100 stimulus presentations. Repetitions of words were randomly dispersed across the list.

\section{Pracedure:}

First the training session was given. Stimulus material was similar to that described in the previous chapter. Initial exposure duration in the training session for the first block was 500 ms. For each block of ten words a different starting exposure time was computed, depending on the performance of the subject in the previous block. During the first block of ten words stimulus exposure time was reduced by $40 \mathrm{msec}$ at the presentation of a new word. For the next four blocks of ten words stimulus presentation for each word were reduced by resp. 30, 20, 10, and 5 ms. Thus stimulus presentation was "narrowed down" at each consecutive block. After the last block of ten words the presentation duration was computed which rendered a probability of correct identification of approximately $40 \%$. The resulting stimulus presentation duration served as the stimulus exposure time for the test session. This duration remained constant during the test session of the experiment. The main difference concerning the stimulus presentation was the feedback procedure: If the subject could not read the word or made a mistake, visual feedback was given; the stimulus was repeated on the screen and disappeared half a second after the onset of a response was regilstered by the voice-key. During the test session instructions and word presentation were the same as in the training session.

\section{RESULTS}

Data of the perceptual identification task were analyzed with ANOVA in a $3 \times 2 \times 2$ Group (DAT patients, depressed patients, and normal controls), Frequency (high ws. low). Facilitation (first vs. second presentation of words) design, with repeated measures on the last two factors. In table 7 the results on identification responses of the perceptual identification task are presented "in Figure 4 the facilitation effiects are depicted.

There is no difference between groups on correct identification of high-and lowfrequency words the first time they are presented [resp. $F(2,35)=0.7$ ) and $F(2,35)=$ 0.1]. There is no main effect for group $[F(2,35)=0.5]$, nor for frequency $[F(1,35)=$ 0.11 . All groups exhibit the facilitation effect, $F(1,35)=236.8, p<0.01$, but there is no difference between the groups in the amount of facilitation $[F(2,35)=2.1]$.

Stimulus presentation durations were analyzed with a Kruskal-Wallis one-way analysis of variance by ranks, corrected for ties. This analysis reveals a difference between groups in the amount of time needed to correctly identify $40 \%$ of the words: $H(d f=2)=19.9, p<0.01(D A T:$ mean $=71.1 \mathrm{msec}(S E M=15.5) ;$ DEP: mean $=57.0$ 
Table 7: Mean Percentage of Correctly Identified Words as Function of Frequency (High- and Low-Frequency Words) and Repefition (First and Second Presentation; SEM between parentheses).

\begin{tabular}{l|cccccc} 
& \multicolumn{2}{|c}{ DAT } & \multicolumn{2}{c}{ Depressed } & \multicolumn{2}{c}{ Control } \\
& High & Low & High & Low & High & Low \\
\hline First Presentation & $37.1(4)$ & $43.2(3)$ & $43.5(5)$ & $42.0(6)$ & $38.6(3)$ & $40.7(5)$ \\
Second Presentation & $64.6(5)$ & $60.4(4)$ & $68.0(5)$ & $71.5(5)$ & $71.1(3)$ & $68.9(5)$
\end{tabular}

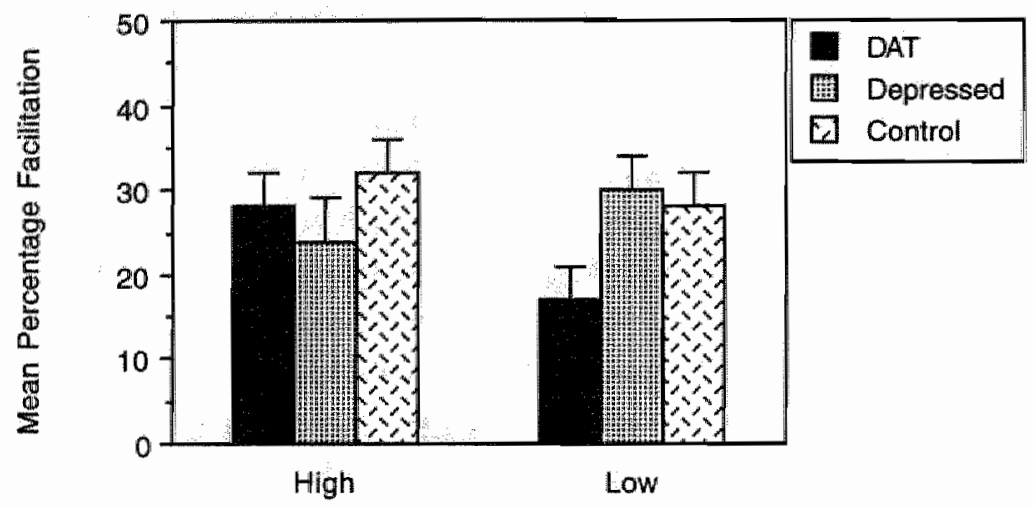

Flgure 4: Mean Percentages Facilitation of DAT Patients, Depressed Patients, and the Control Group as a Funclion of Word-Frequency.

msec $(S E M=5.0) ;$ CON: mean $=31.8$ msec $(S E M=2.7)$ ).

As can be seen in Table 7, the range of facilitation was increased by giving visual feedback but, nevertheless, we did not find any differences in the tacilitation effects of DAT patients, depressed patients and normal elderly subjects. Hence, we were again unable to find support for the hypothesis that DAT patients are impaired on implicit tasks which mainly rely on automatic processing (Salmon et al., 1988; Shimamura et all., 1987; Strauss et al., 1985). 


\subsection{PERCEPTUAL IDENTIFICATION OF WORDS IN SIX AGE-GROUPS (EXPERIMENT 5)}

\section{METHOD}

Experimental Subjects:

Six age groups were tested for their performance on the perceptual identification task. Subjects were recruited by advertizing in a local paper. Selection procedure and criteria were the same as described in the previous paragraph. All subjects were paid for their participation. All subjects had normal, or corrected-to-normal vision. Demographic data are presented in Table 8. Material and procedure were the same as in experiment 4.

Table 8: Demographic Data of the Six Age Groups.

\begin{tabular}{l|cccccc} 
Age-Group & $\begin{array}{c}20-30 \\
(n=13)\end{array}$ & $\begin{array}{c}30-40 \\
(n=10)\end{array}$ & $\begin{array}{c}40-50 \\
(n=10)\end{array}$ & $\begin{array}{c}50-60 \\
(n=11)\end{array}$ & $\begin{array}{c}60-70 \\
(n=10)\end{array}$ & $\begin{array}{c}70-80 \\
(n=13)\end{array}$ \\
\hline Mean Age (years) & $24.6(2)$ & $35.5(2)$ & $44.3(2)$ & $54.6(2)$ & $65.3(1)$ & $75.0(2)$ \\
Male & 6 & 5 & 5 & 5 & 5 & 6 \\
Female & 7 & 5 & 5 & 6 & 5 & 7 \\
Education & $12.7(3)$ & $11.4(3)$ & $12.6(4)$ & $10.6(3)$ & $10.4(3)$ & $8.5(3)$
\end{tabular}

\section{RESULTS}

Data of the perceptual identification task were analyzed with an ANOVA in a $6 \times 2 \times 2$ Group (Age: 20-30, 30-40, 40-50, 50-60, 60-70, and 70-80 years), Frequency (high vs. low), Facillitation (first vs. second presentation of words) design, with repeated measures on the last two factors. In Table 9 the results of the identiflication responses on the perceptual identification task are presented, in Figure 5 the facilitation effects are shown.

There is no difference between groups in correct identification of high- and lowfrequency words the first time they are presented [resp. $F(5,66)=2.1$ and $F(5,66)=$ 1.3]. There is no difference in correct identification of words between the groups [F(5, $61)=2.1]$. High-frequency words are Identified as readily as low-frequency words $[F(1,61)=0.1]$. Words are identified better at the second presentation than at the first presentation, $F(1,61)=632.8, p<0.01$. However, the age-groups do not differ in identification performance $[F(5,61)=1.5]$. Stimulus presentation duration was analyzed by a Kruskal-Wallis one-way analysis of variance by ranks, corrected for ties: Elderly subjects require more time to correctly identify the stimuli at a $40 \%$ at first presentation, $H(d f=5)=30.2, p<0.01$. The mean stimulus presentation durations for the six age groups were: Age 20-30 y: 20.0 (SEM $=1.0)$ msec; $30-40$ y 26. (SEM $=$ 2.6) msec; 40-50 y: 27.0 (SEM = 2.5) msec; $50-60$ y: 33.2 (SEM = 3.0) msec; 60-70 y: $28.0(\mathrm{SEM}=1.9) \mathrm{msec} ; 70-80 \mathrm{y:} 33.1(\mathrm{SEM}=3.2) \mathrm{msec}$. 
Table 9. Mean Percentage of Correctly Identffied Words as a Function of Frequency (High-and Low-Frequency Words) and Repetition (First and Second Presentation; SEM between parentheses).

\begin{tabular}{l|cccccc} 
Age-Group & $20-30$ & $30-40$ & $40-50$ & $50-60$ & $60-70$ & $70-80$ \\
\hline High & & & & & & \\
First Presentation & $35.8(4)$ & $41.0(3)$ & $39.5(4)$ & $29.1(3)$ & $39.0(4)$ & $42.7(3)$ \\
Second Presentation & $75.8(4)$ & $77.0(9)$ & $71.0(4)$ & $62.7(6)$ & $75.0(2)$ & $73.1(4)$ \\
Low & & & & & & \\
First Presentation & $36.2(3)$ & $40.5(5)$ & $42.5(4)$ & $30.9(3)$ & $41.0(5)$ & $42.7(4)$ \\
Second Presentation & $74.6(5)$ & $76.0(3)$ & $74.0(4)$ & $63.6(4)$ & $75.0(3)$ & $68.5(53)$
\end{tabular}

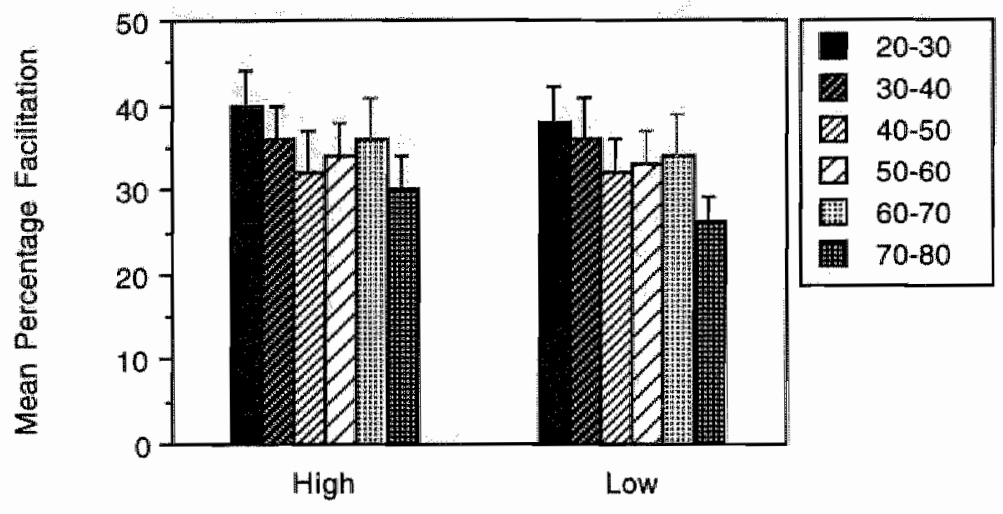

Figure 5: Mean Percentages Facilitation of the Six Age-Groups as a Function of Word. Frequency (High, and Low).

The results of this experiment are contrary to what we expected. Although a slight reduction in the facilitation effect over the age groups can be seen, this reduction does not reach significance. This is in contrast to the age-dependent impairment in the facilitation effect we found in experiment 2 with a perceptual identification task in which auditory feedback was given. Providing visual feedback induces perhaps other processing of the stimuli than giving auditory feedback. We will address this issue in the discussion of this chapter. 


\subsection{PERCEPTUAL IDENTIFICATION AND RECOGNITION OF WORDS AND NONWORDS IN THREE AGE-GROUPS (EXPERIMENT 6)}

To explore whether the deficit observed in our elderly samples in Chapter 2 can be attributed to a deficit in episodic processing we performed the present experiment. Our assumption in this experiment was that the lacilitation effect in the auditory feedback task resulted as a consequence of a single, very short visual presentation of the stimulus, and that auditory feedback would add nothing to the facilitation effect. This was based upon the observation of Jacoby and Dallas (1981, exp. 6) that changing modality between study and test largely abolished facilitation. Therefore, the agerelated impairment on the auditory task should be a consequence of impaired automatic processing of episodic information. The observed increased facilitation effect in the visual feedback task could be the result of feedback serving as an extra presentation within the same modality. However, the finding of preserved priming of the elderly on this task would mean that elderly subjects compensate in one way or another for deficit in automatic episodic memory processing. As is mentioned in the introduction, ageing could parallel the effects of amnesia. If the presence of semantic cues is necessary for preserved priming performance for older adults as it for amnesic subjects, then a possible compensatory mechanism in the visual feedback task could be that the extra presentation allows elderly subjects to process the stimulus at a deeper (semantic) level because visual feedback allows them more processing time. Hence, eliminating semantic processing by presenting nonwords should result in reduced priming in the elderly.

\section{METHOD}

\section{Experimental Subjects:}

Three age-groups were tested on perceptual identification performance and recognition memory performance. The group of young subjects consisted of four women and eight men with ages varying between 21 and 34 years (Mean $A g e=27.2, S D=4.5$ ). Theil mean education was 12.8 years ( $S D=3.8$ ). The middle-aged group consisted of four women and five men with ages varying between 38 and 66 (Mean $\mathrm{Age}=51.4, \mathrm{SD}=11.2$ ). Their mean education was 10.1 years ( $\mathrm{SD}=$ 1.6). And last ${ }_{0}$ the group of elderly subjects consisted of seven women and five men with ages varying between 73 and 77 (Mean $A g e=75.2, S D=1.5$ ). Their mean education was 8.8 years $(S D=3.5)$. They were paid for their participation. All subjects had normal or corrected-tonormal vision.

\section{Material:}

Apparatus was the same as in experiment 4. Also, the same perceplual identification procedure for words was used as in the experiments 4 and 5 (i.e. base-line procedure, followed by perceptual identification). As nonwords take longer to be identified (Feustel et al., 1983; Salasoo et al., 1985) we introduced a second base-line procedure for nonwords in which a list containing 
50 nonwords was presented. The nonwords were chosen from a Finnish dictionary. These words are meaningless but very well pronounceable for a Dutch-speaking population. The second perceptual identification task consisted of a list containing 25 nonwords which served as fillers and were presented only once, and 25 norwwords which were presented twice. This resulted in a list of 75 stimulus presentations. Word-length of nonwords was three or four letters.

Repetitions of words and nonwords were randomly dispersed across the lists. Also, two lists of 24 items each were made for the recognition tasks. The items of each list consisted of two stimuli, a target which had been presented before in the perceptual identification task, and a distractor. The recognition list with words contained 12 items of each frequency: half of the target items of each frequency had been presented only once in the perceptual identification task, the other half twice. The recognition list of the nonwords contained 12 target items which had been seen once and 12 which had been seen twice.

\section{Procedure:}

The procedure of the experiment was basically the same as in experiment 4 and 5 , with the following exceptions: There were two base-line procedures; two perceptual identification tasks, and two recognition tasks. One for words, and one for nonwords. Half of the subjects received the word tests first, and the other half received the nonword tests first. The order was: base-line procedure, perceptual identification, and recognition task. In both the word and the nonword condition visual feedback was given if a stimulus was incorrectly identified.

In the recognition tasks, subjects were instructed to choose the stimulus they had seen before in the perceptual identification task. If in doubt they were encouraged to choose the stimulus which seemed most probable, and the response was registered.

\section{RESULTS}

Data of the perceptual identification tasks and the recognition tasks were analyzed with ANOVA in a 3 (Group: Young, Middle-Aged, and Old) by 3 (Frequency: High, Low, and None) by 2 (Facilltation: First vs. Second Presentation) design with repeated measures on the last two factors. Tukey post-hoc analyses were performed on the facilitation effects, defined as the difference between identification, or recognition at first and second presentation. Stimulus presentation durations were nonparametrically analyzed with a Kruskall-Wallace analysis of variance by ranks, corrected for ties.

\section{Perceptual Identification.}

The results for the perceptual ldentification tasks are presented in Table 10 (lidentification), and Figure 6 (Facilitation). There is no difference in the percentage correct Identification on first presentation of high-frequency words $[F(2,30)=1.2]$, nor for low-frequency words $[F(2,30)=1.1]$, or for nonwords $[F(2,30)=0.5]$. High-, and low frequency words are more probable to be identified than nonwords, $F(2,69)=$ 20.3, $p<0.01$. Stimuli are more readily identified at second presentation than all first presentation, $F(1,30)=378.0, p<0.01$. As can be seen form Figure 6 , the facilitation effect for nonwords is smaller than for words, $F(2,69)=13.6, p<0.01$. However, the 
groups do not differ in the amount of faclitation $[F(2,30)=1.8]$. Also, none of the interaction terms reaches significance.

Tukey's Studentized Rang (HSD) Test on the facilitation effects for high- and lowfrequency words and nonwords reveals no difference between groups in the amount of facilitation of high-frequency words at the alpha $=0.05$ level, nor a difference in the facilitation effect of nonwords. There is, however, a difference between the young and teh elderly group and between the middle-aged and the elderly group in the amount offacilitation for low-frequency words.

The groups differ in the amount of time needed to correctly identify words at baseline levell, $H(d f=2)=58.2, p<0.01$ (Mean. Young $=20.0 \mathrm{msec}(\mathrm{SEM}=1.2)$, Midile-aged $=28.3 \mathrm{msec}(\mathrm{SEM}=2.0)$, and Old $=31.3 \mathrm{msec}(\mathrm{SEM}=2.4)$. The groups also differ in the stimulus presentation durations of nonwords, $H(d f=2)=20.2, p<0.01$ (Mean: Young $=29.6 \mathrm{msec}(\mathrm{SEM}=1.9) ;$ Middle-aged $=41.1 \mathrm{msec}(\mathrm{SEM}=4.2)$, and $\mathrm{Old}=59.6$ msec $(S E M=10.4)$.

\section{Recognition Memory.}

The results for recognition memory can be seen in Table 11. The groups differ on the percentage of correctly recognized items, $F(2,30)=6.9, p<0.01$. Also, nonwords are recognized less well than high- and low-frequency words, $F(2,60)=38.6, p<0.01$. Words and nonwords which were seen twice in the perceptual identification task are recognized more readily than words and nonwords which were only presented once, $F(1,30)=22.2, p<0.01$. This means that a facilitation effect can be observed for recognition memory. Also, this facilitation effect differs across frequencies, $F(2,60)=$ $3.2, p<0.05$. This is probably due to ceiling effects on the recognition of high-and lowfrequency words.

Table 10: Mean Percentage of Correctly Identified Words (High- and Low-Frequency), and Nonwords as a Function of Repetition (First and Second Presentation; SEM between parentheses).

\begin{tabular}{l|ccc} 
& Young & Midide-aged & Old \\
\hline Hiligh & & & \\
First Presentation & $31.3(4)$ & $33.9(4)$ & $38.8(4)$ \\
Second Presentation & $68.8(4)$ & $68.9(6)$ & $71.3(4)$ \\
Low & & & \\
First Presentation & $34.6(3)$ & $36.7(4)$ & $42.5(5)$ \\
Second presentation & $69.6(5)$ & $73.3(4)$ & $66.3(5)$ \\
No & & & \\
First Presentation & $32.0(3)$ & $26.2(2)$ & $29.0(5)$ \\
Second Presentation & $56.0(5)$ & $42.7(6)$ & $49.3(5)$
\end{tabular}




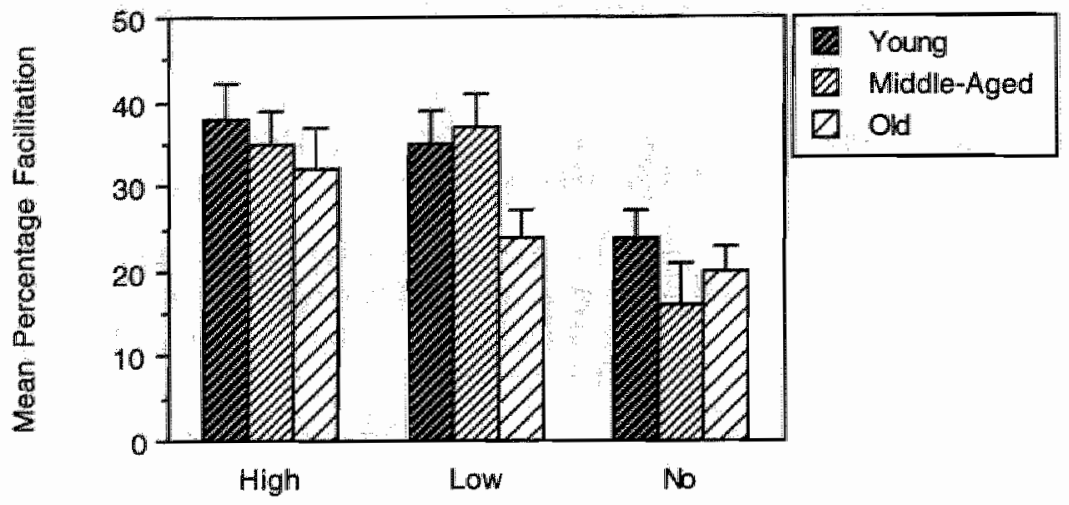

Figure 6: Mean Percentages Facilitation of Words (High-and Low-Frequency), and Nonwords (No) for the Three Age-Groups

Table 11: Mean Percentage of Correctly Recognized Target Items (High- and Low-frequency Words, and Nonwords) as a Function of Repetition (Once or Twice Presented; SEM between Parenthesies).

\begin{tabular}{l|ccc} 
& Young & Middle-aged & Old \\
\hline High & & & \\
Once presented & $90.3(3)$ & $81.5(4)$ & $81.9(4)$ \\
Twice Presented & $94.4(3)$ & $88.9(4)$ & $80.6(5)$ \\
Low & & & \\
Once Presented & $83.3(5)$ & $79.6(5)$ & $70.8(6)$ \\
Twice Presented & $95.8(8)$ & $88.9(4)$ & $84.7(4)$ \\
No & & & \\
Once Presented & $65.3(3)$ & $63.9(2)$ & $54.2(4)$ \\
Twice Presented & $84.7(4)$ & $72.2(8)$ & $68.1(4)$
\end{tabular}

\subsection{DISCUSSION}

The results of the experiments described in this chapter can be summarized as follows: First, increasing the range of the facilitation effect by giving vilsual feedback, and thus avoiding floor-effects, does not result in a difference in facilitation between DAT patients, depressed patients and normal elderly: DAT patients exhibit unimpaired 
priming performance in comparison with depressed patients and normal elderly control subjects. Second, in contrast to what we found in experiment 2 , no age-related impairment can be seen in the amount of facilitation if visual feedback is given after a word has been incorrectly identified. And third, the hypothesis that the age-difference we found in experiment 2 is due to an impaired processing of episodic information is not confirmed: Elderly exhibit the same priming effect for nonwords as do young and middle-aged subjects. On an explicit test of memory there is an age-related impairment.

The results of experiment 5 were in contrast to what we expected. Obviously, changing the mode of feedback between the two tests of perceptual priming differentially affects the age-dependency of the facilitation effect. Our results of the present study are in accordance with the study of Light and Singh (1987, Exp. 3) who found no difference between young and elderly subjects in perceptual identification performance. They are, however, contrary to the results of Davis et al. (1990), notwithstanding the fact that we also tested subjects of above seventy years of age. These authors assert that "[...] elderly can be expected to perform normally on some implicit tasks and to be impaired on other tasks assessing implicit memory. Specifically, whether normal priming occurs in the elderly may depend on subtle differences in task difficulty, [and] the degree to which instructions minimize memory aspects of the task [...]." (p. 296). Our data also suggest that certain task variables are crucial in finding age-related deficits or not on implicit memory performance (for a review of the importance of task variables, see: Richardson-Klavehn \& Bjork, 1988).

It is, therefore, questionable whether implicit memory can be seen as a unitary concept. This issue is also addressed in a study of Hunt \& Toth (1990). They found a functional dissociation between word-fragment completion and perceptual identification, both considered to be implicit measures of memory. (see also: Perruchet \& Baveux, 1989). Witherspoon and Moscovitch (1989) even found stochastic independence between several implicit memory tasks, a criterion which is mostly used as evidence for the existence of two separate memory systems, i.e. implicit vs. explicit memory (Parkin et al., 1990; Shimamura, 1986; Squire et all, 1985). Another purpose of the study of Davis et al. (1990) was to investigate whether a similar pattern (i.e. impaired explicit memory and preserved priming) of the performance of amnesic patients and that of aged subjects could be observed. In contrast to what they found, our present results support this hypothesized pattern. However, Cermak et al. (1985) found no priming effects in amnesic patients on a perceptual identification task in which pseudowords served as stimulus material, while our elderly subjects show normal priming effects for nonwords. Schacter (1985) argues that, for amnesic subjects to show preserved priming, the presence of a semantic cue is essential. In other words, priming performance, at least in amnesic subjects, is dependent upon semantic processing. This is not the case for elderly subjects as is shown by the present data. Moreover, the age-related impairment we found in Chapter 2 cannot be attributed to a general deficit in episodic processing. If this were the case, no priming of nonwords would have been found in the elderly. 


\section{Chapter 4}

\section{A comparison of the effect of different modes of feedback on perceptual identification performance}

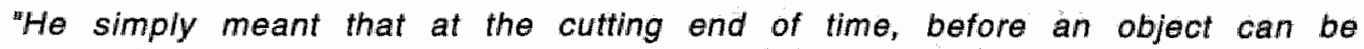
distinguished, there must be a kind of non-intellectual awareness [..]. You can't be aware that you have seen a tree untll after you've seen the tree, and between the instant of vision and the instant of awareness there must be a time lag. We sametimes think of that time lag as unimportant. But there is no justification for thinking that the time lag is unimportant -none whatsoever:"

Robert M. Pirsig: Zen and the Art of Motorcycle Maintenance.

\subsection{INTRODUCTION}

In Chapter 2 (Exp. 1) we tested moderately-to-severely impaired DAT patients and normal elderly control subjects, as well as a group of young and elderiy subjects (Exp. 2), and a group of mildly impaired DAT patients, depressed patients and normal elderly (Exp. 3) on a perceptual identification task in which auditory feedback was given. In Chapter 3, we tested similar experimental groups (Exp. 4 and Exp. 5) on a perceptual identification task in which visual feedback was given. We found an age-difference in the facilitation effect on the task with auditory feedback, but not on the task with visual feedback. On both tasks we could not find any differences between the DAT and depressed patients, nor between patient groups and the control groups. The differential results concerning the age-dependency of the facilitation effect between the two tasks were rather surprising. As we argued in Chapter 2, the observation of an age-related impairment in the facilitation effect on the task with auditory feedback could indicate that there is an decline in automatic memory performance with increasing age. 
A possible explanation for the failure to find an age-related deficit in the task with visual feedback could be that the elderly compensate for their deficit in the task with visual feedback. However, a different mode of feedback was not the only difference between the two tasks. We also used different displays. Letter-size is greater on the screen which consisted of LED-displays. This resulted in much smaller base-line presentation durations in the visual feedback task, as well as in a reduction of the variance in presentation durations. However, changing the apparatus between the auditory and visual feedback tasks may have had different consequences for the facilitation effects we observed in these two types of tasks.

To get a direct comparison between the effect of mode of feedback, we conducted the present experiments in which the screen with the LED-displays was used and the mode of feedback was treated as a within-subject factor. We also added a task in which no feedback was given to explore the importance of feedback. In this way it is possible to get an impression of how much auditory and visual feedback each contribute to the facilitation effect. If stimuli are presented in different modalities at study and test a smaller (Bassili, Smithe \& MacLeod, 1989) or no (Jacoby, 1983a) facilitation effect results. This phenomenon is generally called modality-specificity. Based upon the phenomenon of modality-specificity, and the statement of Hasher and Zacks (1979) that feedback has no additional ameliorating effect upon automatic processes, we assumed that the facilitation effect seen in the auditory feedback task represents the true measure of automatic processing: This facilitation effect results only as a consequence of the single, very short visual presentation. Furthermore, we hypothesized that other factors contribute to the facilitation effect in the visual feedback task, factors that dissimilarly affect facilitation performance of young and older adults. We hypothesized that compensatory mechanisms might act in this task, because the visual feedback represents an additional (unmasked) presentation from which the elderly benefit more so that their performance equals that of the young.

Our expectations concerning the three different perceptual identification tasks are as follows: First, in line with our previous findings, there will be no differences between DAT patients and normal elderly controls on any of the three perceptual identification tasks.

Second, we expect to replicate the findings concerning the age-dependency of the facilitation effects on the visual and the auditory tasks. That is, no age-related impairment on the visual feedback tasks, in contrast to an age-related decline in the fecllitation effect after auditory feedback is given.

And third, we expected to find similar outcomes on perceptual identification after auditory feedback is given, and the task in which no feedback is given. In other words, the facilitation effects of these two tasks should be similar in magnitude, and thus, facilitation on the task without feedback should also show an age-related decrement.

Additionally, we varied the lag between first and second presentation to get an Impression of the persistence of the priming effect. Priming as a consequence of episodic activation is presumed to persist over longer intervals, whereas priming as a result of semantic activation decays rapidly (Cermak et al., 1985; Forster \& Davis, 1984; Jacoby \& Dallas, 1981; Musen \& Treisman, 1990). Should semantic activation (via cross-modal feedback) contribute to the facilitation effect on perceptual identification with auditory feedback, we expect to find a decrease in facilitation performance over increasing lags. 


\subsection{PERCEPTUAL IDENTIFICATION WITH VISUAL, AUDITORY, AND NO FEEDBACK IN DAT PATIENTS AND NORMAL ELDERLY (EXPERIMENT 7)}

\section{METHOD}

\section{Experimental Subjects:}

All DAT as well as all depressed patients from experiment 3 and experiment 4 were approached to participate in the present experiment. Of the depressed patients, only eight volunteered to cooperate. The remaining patients refused co-operation. Thus, the size of the depressive sample is rather small, and data of these patients will not be presented here.

DAT patients. For the initial selection criteria for the DAT patients see the description of the patient groups in experiment 3 and 4 . Of the DAT patients we approached for re-examination, 15 were able and willing to participate in the present experiment. Seven of the remaining patients were approached, but could not be re-examined because they were too impaired: four of them now lived in a nursing home. Four patients were not approached because the interval between first and second examination was only a few months. The interval between the first and the second examination of the tested patients varied between 1.2 and 3 years.

The data of one patient were excluded because of technical problems. Two patients refused to continue after part of the experiment was concluded, so the data of these patients were excluded from the experiment. This resulted in a group of 12 DAT patients. Demographic data are presented in Table 12.

Control Subjects. Selection criteria and procedure of the control subjects were the same as described in previous chapters. They were matched for age, and as far as possible for education to the DAT patients. All subjects were paid for their participation "and had normal "or coirrectedto-normal vision.

\section{Material:}

Apparatus was the same as in the previous experiment. All words presented in the experiment were of intermediate frequency $(6 / 100.000<$ freq. < 90/100.000; Uit den Boogaart, 1975). Words varied in length between three and four letters and were presented in upper-case letters. In the training session 50 words were presented for perceptual identification. Three lists were made for perceptual identification. Each list consisted of 20 words which were presented twice, and 20 words which were presented only once and served as fillers. This resulted in three lists of each 60 stimulus presentations. The lag between the first and the second presentation of words varied between 1 and 20 intervening stimuli. For each perceptual identification test a recognition test list was made. These lists each contained 20 items. Each item consisted af two words: One word which had been presented in the perceptual identification task was paired with a word of 
equal frequency value which had not been presented. Half of the target words had been presented twice. The remaining 10 target words had been presented only once.

\section{Procedure:}

First a training session was giwen. The computation of the base-line presentation duration, as well as the stimulus presentation procedure were the same as in the experiments described in Chapter 3. Then the first perceptual identification task followed, after which the corresponding recognition lask was given. Then the second perceptual identification and recognition test was given, followed by the third. The three perceptual identification tasks differed in the way feedback was given: One with visual feedback, one with auditory feedback and one in which no feedback was given. The visual feedback procedure was the same as in the experiments described in Chapter 3 , the auditory feedback procedure was the same as in Chapter 2. During the perceptual identification task without feedback, nothing was said or done to provide the subjects of information about whether or not the response was correct. instructions included that the subjects were informed if or how they would receive feedback if a word was incorrectly identified. The three test lists were rotated across subjects and experimental conditions. The order of the types of feedback conditions was rotated across subjects, so that each feedback condition was presented first, second, or third an equal amount of times.

Table 12: Demographic Data of the DAT patients and the Control Group.

\begin{tabular}{l|cc} 
& DAT & Controll \\
\hline Male & 8 & 7 \\
Fernale & 4 & 5 \\
Age & $69.0(6)$ & $69.3(6)$ \\
Education & $10.7(4)$ & $7.7(2)$
\end{tabular}

\section{RESULTS}

Data of the three perceptual Identification and recognition tasks were analyzed separately with ANOVA in a 2 (Group) by 2 (Facilitation) design with repeated measures on the last factors. Table 13 (lldentification), and Figure 7 (Facilitation) show the results of the perceptual identification tasks, Table 14 that of the recognition tasks.

The analysis of the factor Lag was as follows: First we counted the number of facilitated items from lag 1 to 5 (Block 1), from lag 6 to 10 (Block 2), from lag 11 to 15 (Block 3), and lag 16 to 20 (Block 4). Subsequently, we analyzed these data with ANOVA in a 2 (Group) by 4 (Block) design with repeated measures on the last factor. The number of facilitated items per block was only analyzed for the visual and auditory 
Table 13: Mean Percentage Correctly Identified Words as a Function of Mode of Feedback (Visual, Auditory, or No Feedback) and Repetition (First and Second Presentation; SEM between Parentheses).

\begin{tabular}{l|cc} 
& DAT & Control \\
\hline Visual Feedback & & \\
First Presentation & $39.2(7)$ & $39.2(4)$ \\
Second Presentation & $58.8(7)$ & $63.8(5)$ \\
Auditory Feedback & & \\
First Presentation & $31.7(6)$ & $38.3(4)$ \\
Second presentation & $34.2(6)$ & $50.4(6)$ \\
No Feedback & & \\
First Presentation & $29.6(6)$ & $28.8(4)$ \\
Second Presentation & $27.5(5)$ & $32.1(4)$
\end{tabular}

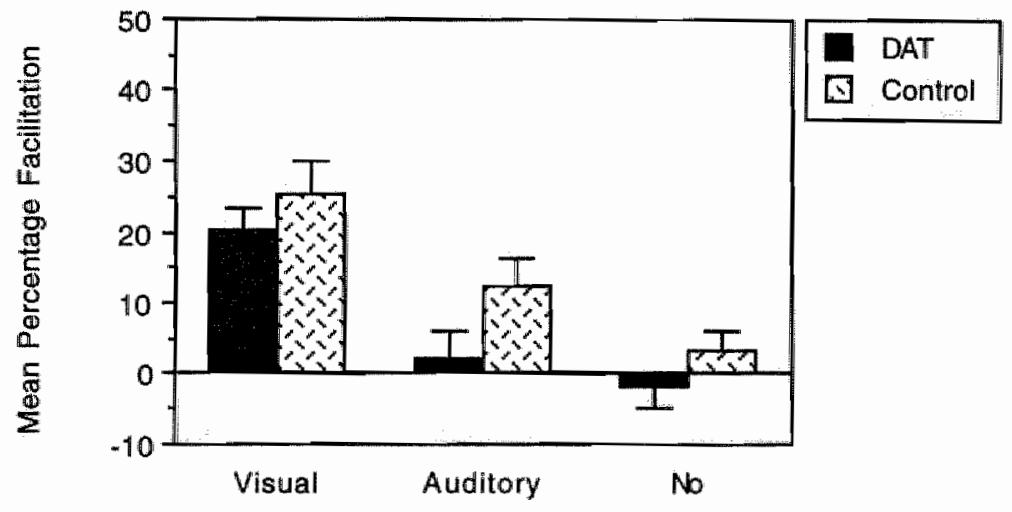

Figure 7: Mean Percentages Facilitation of Words after Visual, Auditory or No Feedback.

feedback tasks, because no facilitation effects were observed in the No-feedback condition. The outcomes of this analysis are represented in Figure 8.

Perceptual identification with visual feedback results in a similar amount of facilitation for DAT patients and the healthy older adults $[F(1,22)=0.7]$. The number of facilitated items stays the same as the lag between first and second presentation is increased $[F(3,66)=1.6]$, and both DAT patients and controls show the same patterm with increasing lag $[F(3,66)=0.2]$. There is also a facilitation effect for recognition memory, $F(1,22)=19.3, p<0.01$. DAT patients, however, recognize fewer items as normal elderly subjects do, $F(1,22)=5.3, p<0.05$.

The results of perceptual identification after auditory feedback is given again no show 

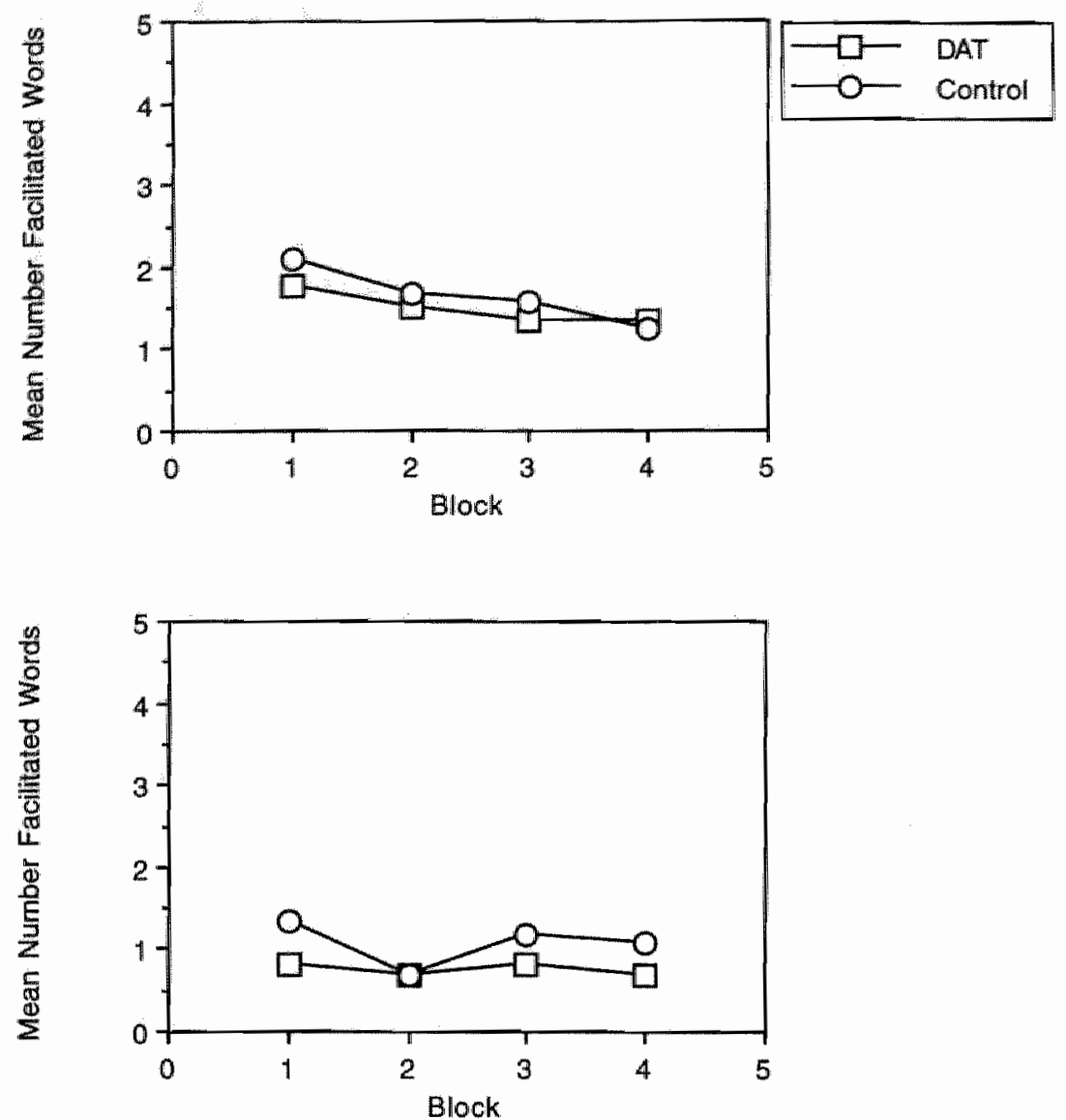

Figure B: Number of Facilltated Words as a Function of Lag after Visual (above) or Auditory (below) Foedback (Block $1=\operatorname{Lag} 1 \cdot 5$; Black $2=$ Lag 6-10; Block $3=11-15$; Block $4=$ Lag 16 20). 
Table 14: Mean Percentage Correctly Recognized Target Items as a Function of Mode of Feedback(Visual , Auditory, or No Feedback) and Repetition (Once or Twice Presented, SEM between Parentheses).

\begin{tabular}{l|cc} 
& DAT & Control \\
\hline Visual Feedback & & \\
Once Presented & $65.0(6)$ & $76.7(3)$ \\
Twice Presented & $76.6(4)$ & $88.3(2)$ \\
Auditory Feedback & & \\
Once Presented & $56.7(7)$ & $75.0(4)$ \\
Twice Presented & $71.7(7)$ & $89.2(4)$ \\
No Feedback & & \\
Once Presented & $42.5(5)$ & $55.8(3)$ \\
Twice Presented & $55.8(4)$ & $60.8(5)$
\end{tabular}

difference between the magnitude of the facilitation effect of DAT patients and their controls $[F(1,22)=2.6]$, although individual $T$-tests comparing differences in identification performance between first and second presentation in each group reveal that the normal elderly exhibit a facilitation effect, $T(11)=2.9, p<0.05$, while the DAT patients do not $[T(11)=0.6]$. Increasing the lag does not result in a decline of the number of facilitated items $[F(3,66)=1.1]$, nor do the DAT patients and the control subjects differ on this aspect $[F(3,66)=0.4]$. The analysis of the data on recognition memary (See Table 14) show a facilitation effect, $F(1,22)=10.0, p<0.01$. Again, DAT patients recognize fewer items, $F(1,22)=7.8, p<0.01$.

Surprisingly, neither DAT patients, nor elderly controls benefit from prior presentation on perceptual identification if no feedback is given, that is, no facilltation effect arises on this task $[F(1,22)=0.1]$. As no facilitation effect for the task without feedback could be observed we did not analyse the effect of lag. Also, none of the groups recognizes the items above chance, with the exception that elderly controls recognize items better than chance if they have been presented twice. This results in a difference on recognition memory performance between the two groups, $F(1,22)=4.2$, $\mathrm{p}<0.05$.

Stimulus presentation durations were analyzed by a Mann-Whitney U-test corrected for ties: As in our previous experiments, DAT patients require more time than control subjects to correctly identify $40 \%$ of the words at first presentation (resp.: mean = $82.9 \mathrm{msec}, \mathrm{SEM}=38.1$, and mean $=31.3 \mathrm{msec}, \mathrm{SEM}=3.0 ; z=3.8, p<0.01$ for a twotailled test). 


\subsection{PERCEPTUAL IDENTIFICATION WITH VISUAL, AUDITORY, AND WITHOUT FEEDBACK IN YOUNG, MIDDLE-AGED, AND ELDERLY SUBJECTS (EXPERIMENT 8)}

\section{METHOD}

\section{Experimental Subjects:}

Three age groups were tested. Siubject selection and recruitment procedures were the same as in previous experiments. Demographic data are presented in Table 15.

Material and Procedure:

Material and procedure were identical to that of experiment 7 .

Table 15: Demographic Data of the Three Age-Groups

\begin{tabular}{l|ccc} 
& $\begin{array}{c}\text { Young } \\
(\mathrm{n}=15)\end{array}$ & $\begin{array}{c}\text { Middlle-aged } \\
(\mathrm{n}=10)\end{array}$ & $\begin{array}{c}\text { Old } \\
(\mathrm{n}=11)\end{array}$ \\
\hline Male & 8 & 7 & 4 \\
Femalle & 7 & 3 & 7 \\
Age & $19.4(2)$ & $61.3(9)$ & $75.3(5)$ \\
Education & $11.6(2)$ & $9.3(3)$ & $6.9(2)$
\end{tabular}

\section{AESULTS}

Data of the present experiment were analyzed in the same way as in experiment 7 . The results for the perceptual identification tasks are represented in Table 16 (Identification), and Figure 9 (Facilitation), those on recognition memory performance in table 17. The effect of Lag is illustrated in Figure 10.

In the visual feedback task a facilitation effect can be observed, $F(1,33)=72.3, p<$ 0.01 . As we expected there is no difference between groups in the amount of facilitation $[F(2,33)=1.9]$. A similar number of items is facilltated at all lags $[F(3,99)=$ $0.3]$, and the groups do not differ in this aspect $[F(6,99)=0.4]$. Recognition memory, after the visual feedback task reveals no difference in performance between the three groups $[F(2,33)=0.2]$. Nor does a facilitation effect arise for recognition memory $[F(1,33)=2.7]$.

In contrast to what we found for perceptual identification after visual feedback was given, the age groups do differ in the amount of facilitation on the task in which auditory feredback is given, $F(2,33)=3.6, p<0.05$. Lag does not affect the number of facilitated items $[F(3,99)=0.7]$, and this pattern is similar for all age-groups $[F(6,99)$ 
Table 16: Mean Percentage Correctly ldentified Words as a Function of Mode of Feodback (Misuall, Auditory, or No Feedback) and Repetition (First and Second Presentation $\mathrm{SEM}$ between Parentheses).

\begin{tabular}{|c|c|c|c|}
\hline & Young & Middle-aged & Old \\
\hline \multicolumn{4}{|l|}{ Visuall Feedback } \\
\hline First Presentation & $40.7(4)$ & $38.0(4)$ & $35.5(6)$ \\
\hline Second Presentation & $72.0(4)$ & $56.0(5)$ & $59.1(7)$ \\
\hline \multicolumn{4}{|l|}{ Auditory Feedback } \\
\hline First Presentation & $3.4 .3(6)$ & $41.0(4)$ & $31.8(7)$ \\
\hline Second presentation & $52.3(5)$ & $60.5(5)$ & $40.0(7)$ \\
\hline \multicolumn{4}{|l|}{ No Feedback } \\
\hline First Presentation & $33.0(5)$ & $30.5(5)$ & $33.2(7)$ \\
\hline Second Presentation & $35.7(5)$ & $36.5(4)$ & $34.1(7)$ \\
\hline
\end{tabular}

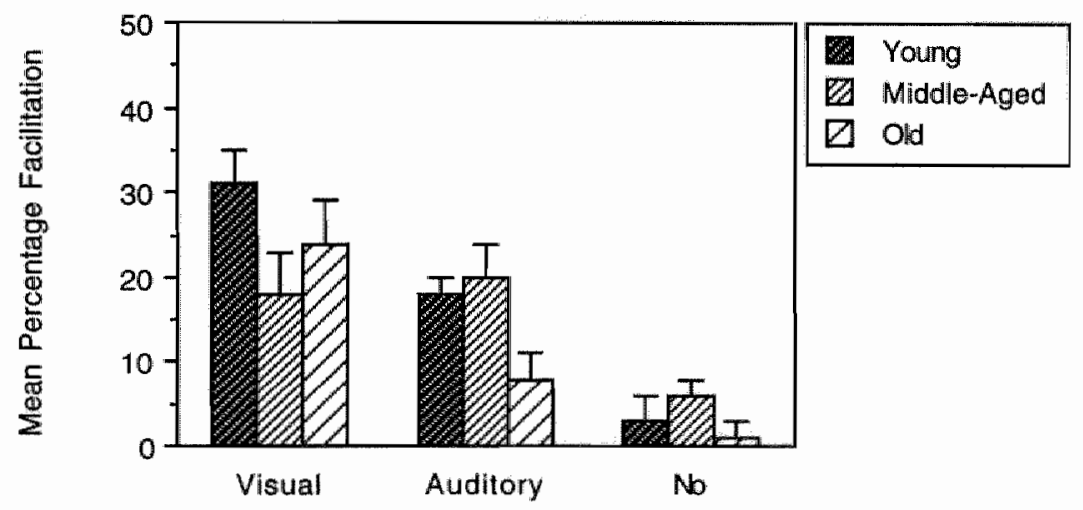

Figure 9: Mean Percentages Facilitation of Words after Visual "Auditory, or No Feedback.

$=1.6]$. After the auditory feedback task the groups also differ on the recognition of target words, $F(2,33)=5.5, p<0.01$. On this task a faclitation effect can be observed for recognition memory, $F(1,33)=22.8, p<0.01$, but there is no interaction between age and the amount of facilitation $[F(2,33)=0.3]$.

On the task in which no feedback is given, there is an effect of repeated presentation, $F(1,33)=4.3, p=0.05$. However, individual $T$-tests reveal that the observed facilitation effect is mainly due to the performance of the middle-aged, as only these subjects exhibit a benefit from repeated presentation, $T(9)=2.4, p<0.05$. Both the young and the elderly do not [resp: $T(14)=0.9$, and $T(10)$ is 0.4 ]. Nevertheless, no interaction between age and facilitation can be found $[F(2,33)=0.9]$. Recognition memory after the perceptual identification task without feedback shows a 
difference between groups in recognition of target items, $F(2,33)=5.1, p=0.01$. Individual T-tests show that the middle-aged and the elderly groups perform at chance level in recognizing litems that have only been presented once in the perceptual identification task without feedback [Resp: $T(9)=0.7$, and $T(10)=1.1$ ]. A facilitation effect can also be observed, $F(1,33)=8.3, p<0.01$. However, there is no interaction between group and facllitation $[F(2,33)=0.4]$. Stimulus presentation duration was nonparametrically analyzed by a Kruskal-Wallis one-way analysis of variance by ranks, corrected for ties. The three groups differ in the amount of time they require to correctly identify $40 \%$ of the stimuli at first presentation: $H(d f=2)=22.22, p<0.01$ (Young: mean $=19.0$ msec, $S E M=1.4$; Middle-aged: mean $=26.0$ msec, $S E M=1.9$; Old: mean $=37.3 \mathrm{msec}, \mathrm{SEM}=2.3$ ). 

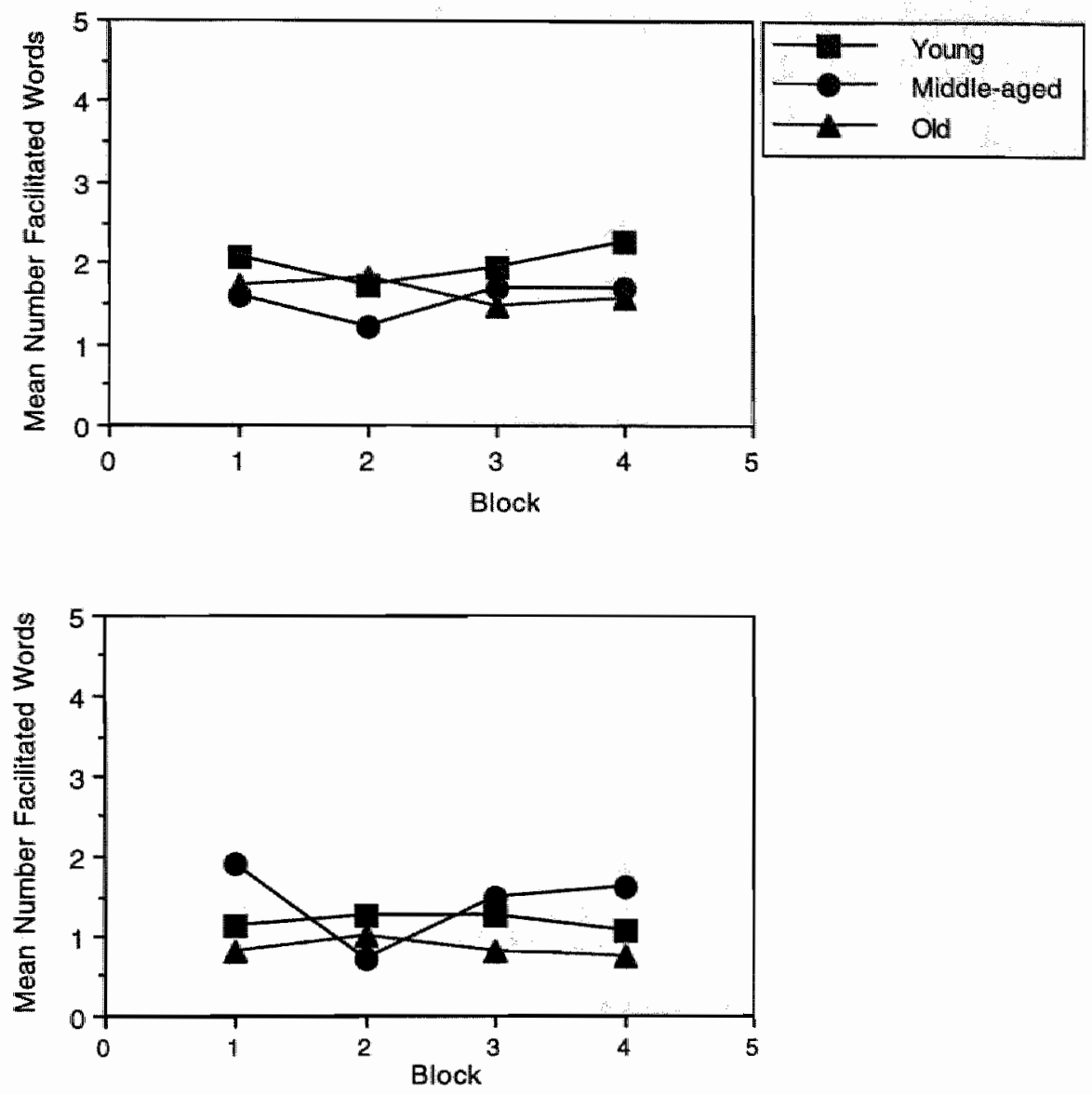

Figure 10: Number of Facilitated Words as a Function of Lag after Visual (above) or Auditory (below) Feedback (Block 1= Lag 1-5; Block $2=\operatorname{Lag} 6-10$; Block $3=11-15$; Block $4=$ Lag 1620). 
Table 17: Mean Percentage Correctly Recognized Target Items as a Function of Mode of Feedback (Vistuall, Auditory, or No Feedback) and Repetition (Once or Twice Presented; SEM between Parentheses).

\begin{tabular}{l|ccc} 
& Young & Middle-aged & Old \\
\hline Visuall Feedback & & & \\
Once Presented & $86.0(2)$ & $81.0(4)$ & $78.2(4)$ \\
Twice Presented & $83.3(6)$ & $90.0(3)$ & $87.3(3)$ \\
Auditory Feedback & & & \\
Once Presented & $83.3(4)$ & $81.0(6)$ & $71.8(4)$ \\
Twice Presented & $98.0(1)$ & $94.0(3)$ & $81.8(5)$ \\
No Feedback & & & \\
Once Presented & $66.0(5)$ & & $54.0(4)$ \\
Twice Presented & $76.7(4)$ & $67.0(5)$ & $60.9(4)$
\end{tabular}

\subsection{DISCUSSION}

The major finding of the present study is that the results of the experiments described in Chapter 2 and 3 can be replicated: First, there is no difference in performance between DAT patients and normal elderly in the facilitation effect on the perceptual identification tasks with visual and auditory feedback. And second, there is no agerelated impairment in performance on the task with visual feedback, in contrast to the age-related decrement in the magnitude of the facilitation effect which can be observed on the auditory feedback task.

The results of the perceptual identification task in which no feedback is given, however, stand in contrast to what we expected. We anticipated to find a similar facilitation effect on the auditory feedback task and the task without feedback. The reason why we made this assumption was that cross-modal (i.e. auditory) feedback should add little or nothing to the facilitation effect resulting from the single, short visual first presentation of an item. Consequently, we hypothesized that an age-related impairment should be observed on both tasks.

However, none of the groups we tested, with the exception of the middle-aged group in experiment 8 , showed a facilitation effect in the task without feedback. Presenting an item for a very short duration seems not enough for the formation of an episodic trace: for most subjects, feedback about the correctness of a response seems a necessary prerequisite for a facillitation effect to occur. It is, however, not clear why the middle-aged group forms an exception.

Salasoo et al. (1985), proposed a two factor model (See: Figure 11) on perceptual identification performance which might offer an explanation. In their model, the first factor involves the existence of "codes". Activation of pre-existing codes is responsiblle for the rapid (automatic) identification of words. The second factor involves the existence of episodic images which evolve based upon the analysis of context cues. Activation of these episodic images is a necessary prerequisite for a facilitation effect 
to emerge. Both activation of codes as well as the episodic images feedback on the letter and feature level of analysis. They argue ".] that for episodic images to facilitate identification, it is necessary that appropriate context cues be used to probe memory. Thus, when context cues are unavailable [.] facilitation will decrease or even disappear." (p. 67) It is possible that the very short duration with which the stimuli are presented in the task without feedback is long enough for the fast (automatic) activation process of the "codes", but not long enough for the formation of episodic images. Thus, identification performance at second presentation is similar to that at first presentation. Facilitation does not occur because no (or incomplete) episodic images exist and thus no interaction can be observed between the activation of the codes and the contribution of the episodic images. In line with this model, a possible explanation for facilitation in the auditory feedback task would be that episodic images are formed through the feedback route, or through the formation of context cues provided by the auditory feedback. Thus, the age-related decrease in facilitation in the auditory feedback task could result either because feedback from activation of the codes does not occur or, at least to a lesser extent than in the young. Or, alternatively, that auditory feedback does not yield enough context cues to the elderly to result in a strong enough episodic image.

The first possibility is in accordance with the assumption (Balota \& Duchek, 1988; Burke et al., 1987; Rabbit, 1984) that elderly subjects suffer from semantic encoding deficits (e.g. problems in gaining access to semantic memory). Therefore it is presumed that most of the impairments observed on tests of episodic memory are attributable to the sensitivity of those tasks to semantic processing. This would also explain why we find preserved performance on the task in which nonwords are used as stimuli, as no semantic representation exists for these words, and hence access to semantic structures is irrelevant.

The second possibility is in agreement with the evidence described by Light (1991). In her review she comes to the conclusion that evidence for several theories about the memory impairments in old age is often contradictory, or scarce. However, the only constant factor on which memory impairments can be found is that on context processing (see also Craik, 1983, 1986). Older adults come to rely more upon the presence of context cues. If these context cues are unavailable memory impairments arise in the elderly.

Concerning the results of recognition memory the following can be observed: On all tasks DAT patients recognize fewer words than normal elderly. Recognition performance after the task without feedback is hardly above chance, especially for the DAT group. In experiment 8 , no age-related impairment in recognition can be seen after visual feedback is given, but there is an age-related impaiment after the auditory task and the task without feedback. After the perceptual identification without feedback, the young perform well above chance, even after only one presentation. The middle-aged and the elderly recognize items which were presented only once at chance level. After two presentations, however, they recognize the items above chance. This offers an interesting issue about the relation between perceptual identification and recognition memory: It is possible that recogniltion memory can be observed in the absence of a facilitation effect. These differential results for perceptual identification and recognition memory can be added to the long list of evidence concerning a dissociation between implicit and explicit memory measures. 


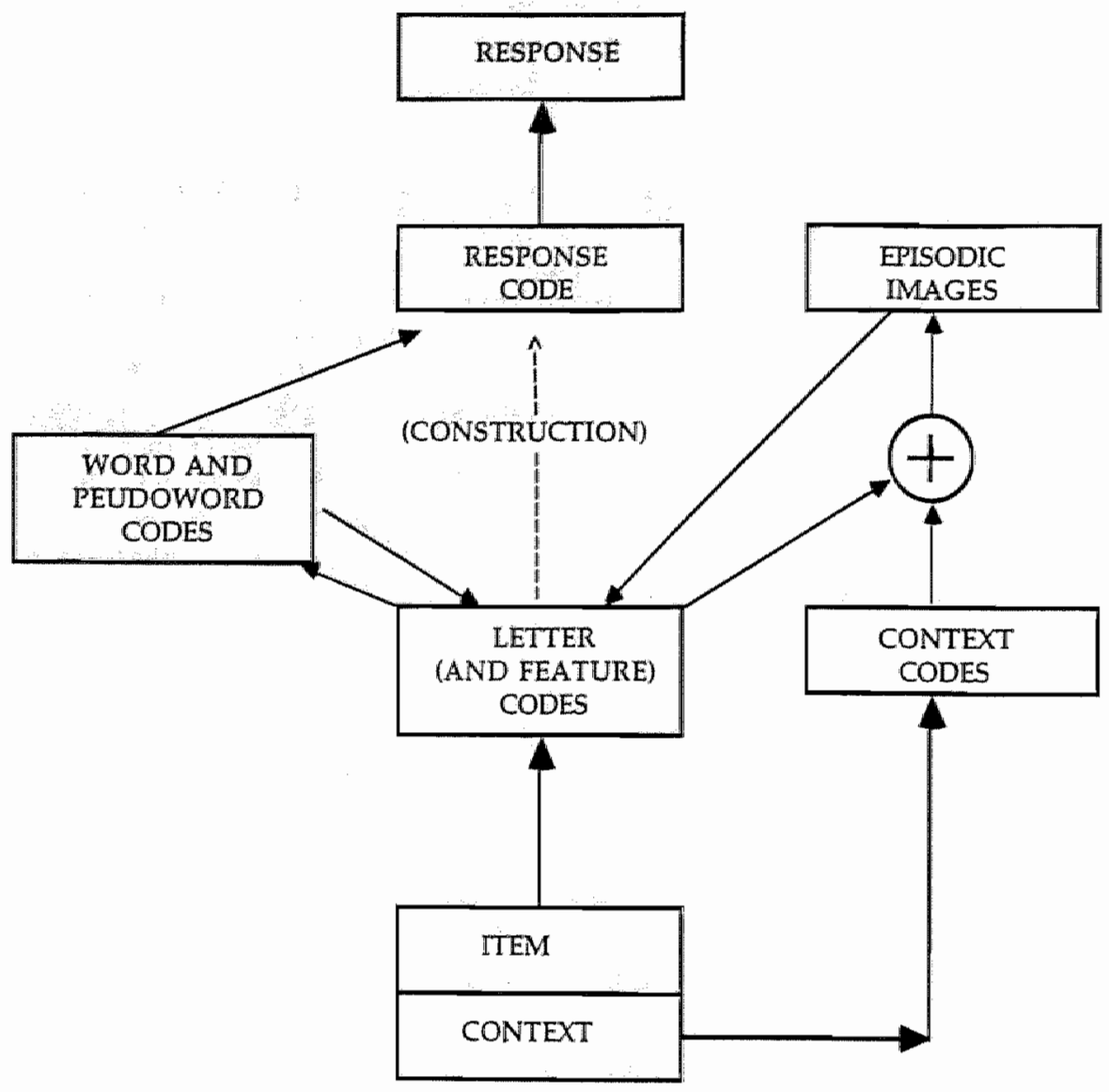

Figure 11: Representation of the Salasoo et al. (1985) model. 


\section{Chapter 5}

\section{The facilitation effect after three different intervals between study and test.}

"Real memory, at the cost of much effort, learns to remember but not to forget". Umberto Eco: Foucault's Pendulum.

\subsection{INTRODUCTION.}

In the previous chapter we proposed that the age-related impairment on the auditory feedback task arises, 1) either because auditory feedback does not provide as many contextual cues at retrieval as visual feedback does. Possibly, older adults are more sensitive to the amount of contextual information that is available. Or 2), elderly subjects exhibit a reduced facilitation effect in the auditory feedback task as a result of a deficit in semantic processing. These possibillties will be further explored in the following chapter. But first a comparison will be made of the specific task variables in the three feedback tasks to clarify the line of reasoning.

Similar Findings Across The Experimental Groups.

In figure 12 the procedure that was used in the three feedback tasks is schematically represented.

- As can be seen in this schematic representation, first and second presentations of stimult are the same, not only within each procedure but also across procedures.

- However, the results of the No-Feedback condition indicate that a single, short, masked presentation of stimuli does not lead to a facilitation effect. 
- Therefore, it may be concluded that a facilitation effect only arises if feedback is given. Also, given the observation that the first presentation is the same in all three procedures, it may be assumed that it does not contribute to the facilitation effect in any of the tasks. Thus, feedback can be considered to be the first presentation to contribute to a facilitation effect. In the next paragraphs, the presentation of feedback will be considered to be the first presentation, and will be designated as such.

- The way in which feedback is given matters for the observed amount of facilitation. Auditory feedback results in less facilitation than visual feedback. So the question is: what constitutes the difference between the two procedures?

- They differ in two ways. First, in the auditory feedback procedure there is a modality change between first (note: feedback) and second presentation, whereas in the visual feedback procedure modality of presentation is constant. The second difference lles in the duration of the first presentation. In the auditory task presentation duration is rather constantly limited. The word is presented for as long as it takes the experimenter to pronounce the word. In the visual feedback procedure the word disappears from the screen only after the subject has read it aloud, so ample time is provided to analyze the stimulus and its characteristics.

To summarize: There are two differences in task variables that may account for thedifference in the amount of facilitation between the feedback tasks, which can be observed in all experimental groups:

1) modality (constant vs. changed),

2) stimulus presentation duration (ample vs. limited).

The findings obtained thus far indicate an age-associated difference in sensitivity to these task variables. So, either elcerly people are more sensitive to the change in modality. Or they are more sensitive to manipulations in stimulus presentation duration. That is, limiting the stimulus presentation duration at encoding is disadvantageous for the elderly groups.

Introducing The Interval Task.

In the experiments which will be presented below the persistence of the episodic image underlying the facilitation effect will be further explored in each experimental group. Measuring the consistency is accomplished by introducing a delay between study and test. To ensure optimal conditions for the elderly to form episodic images, words are:

1) presented within the same modality at study and test,

2) presented for as long as it takes the subject to read them aloud at first presentation,

3) presented unmasked at first presentation.

(Note that study conditions are thus similar to the feedback procedure used in the visual feedback task, in which no age-related deficit was found)

Hence, testing implicit memory for words immediately after study is completed (interval $=0$ min.), the same amount of facilitation as was observed in the visual feedback task is expected for all experimental groups. In other words: Neither age-related impairments, nor DAT-related impairments are expected to occur at the shortest interval. 
The strength of the episodic image can be assessed by increasing the interval between study and test and measuring the facilitation effect at each delay. Episodic activation has been reported to persist over hours to days (Musen \& Treisman, 1990), even up to a year (Kolers, 1976). By introducing a delay, also an impression can be gained about the relative contribution of semantic processing. Semantic activation fades quickly (Cermak et al, 1985). These authors found Korsakoff patients, who are known to exhibit gross episodic memory deficits (Diamond \& Rozin, 1984; Schacter, 1985; Schacter \& Graf, 1986b) to be unimpaired on an immediate test of implicit memory, but they showed almost no retention at all when implicit memory was tested at the longer delays. They reasoned that performance on the immediate test must be based upon semantic activation which is presumed to be intact in these patients (Kinsbourne \& Wood, 1982; Schacter, 1985). Possibly, DAT patients show a similar pattern (i.e. unimpaired performance at the immediate test based on semantic processing which fades quickly, but no facilitation effects at the longer intervals).

In paragraph 5.2 the results will be presented of an experiment in which DAT patients and normal elderly are compared on their performance on the interval task.

Thus far, no differences between performance of DAT patients and healthy elderly have been found. It is, however, possible that facilitation performance of these patients is similar to that of age-matched controls, but sustained by or based on different processes such as seems to be the case in amnesic Korsakoff patients.

On the other hand, even if underlying processes are presumed to be similar in DAT patients and normal elderly, it is also possible that DAT patients forget information at a faster rate. The studies of Hart et al. (1987c), and Moss et al. (1986) lend some support for this notion. Faster forgetting in the perceptual identification task should be revealed by a steeper decay of the facilitation effect over time of DAT patients when compared to healthy elderly. 


\begin{tabular}{|c|c|c|c|}
\hline & $\begin{array}{l}\text { Auditory Feedback } \\
\text { Task }\end{array}$ & $\begin{array}{l}\text { No Feedback } \\
\text { Task }\end{array}$ & $\begin{array}{l}\text { Visual Feedback } \\
\text { Task }\end{array}$ \\
\hline $\begin{array}{l}\text { First } \\
\text { Presientation }\end{array}$ & $\begin{array}{l}\text { masked } \\
\text { - indual modality } \\
\text { assessed } \\
\text { stimulus duration }\end{array}$ & $\begin{array}{l}\text { masked } \\
\text { - visual modality } \\
\text { - individually } \\
\text { assessed } \\
\text { stimulus duration }\end{array}$ & $\begin{array}{l}\text { - masked } \\
\text { - visual modality } \\
\text { - individually } \\
\text { assessed } \\
\text { stimulus duration }\end{array}$ \\
\hline Feedback & $\begin{array}{l}\text { - unmasked } \\
\text { auditory modality } \\
\text { stimulted } \\
\text { lims duration }\end{array}$ & & $\begin{array}{l}\text { - unmasked } \\
\text { - visulall modality } \\
\text { - unlimited } \\
\text { stimulus duration }\end{array}$ \\
\hline \multirow[t]{2}{*}{$\begin{array}{l}\text { Second } \\
\text { Presentation }\end{array}$} & $\begin{array}{l}\text { - masked } \\
\text { - isual modality } \\
\text { assessed } \\
\text { stimulus duration }\end{array}$ & $\begin{array}{l}\text { - masked } \\
\text { - visual modality } \\
\text { - individually } \\
\text { assessed } \\
\text { stimulus duration }\end{array}$ & $\begin{array}{l}\text { - masked } \\
\text { - visual modality } \\
\text { - individually } \\
\text { assessed } \\
\text { stimulus duration }\end{array}$ \\
\hline & Facilitation & Facilitation (0) & Facilitation $(++)$ \\
\hline
\end{tabular}

Figure 12: Schematic Representation of the Different Feedback Procedures Used in the Various Experiments Previously Presented. 


\subsection{A COMPARISON OF THE PERFORMANCE OF DAT PATIENTS AND NORMAL ELDERLY SUBJECTS ON PERCEPTUAL IDENTIFICATION AFTER THREE DIFFERENT DELAYS (EXPERIMENT 9)}

\section{METHOD}

\section{Experimental Subjects:}

All fifteen DAT patients that were approached for experiment 4.2 were tested. Also, the same twelve elderly control subjects were tested. Three additional control subjects were matched in age and education to the three DAT patients whose data were excluded from the analyses in experiment 7. Demographic data are presented in Table 18.

Table 18: Demographic Data of the DAT Patients and the Control Group.

\begin{tabular}{l|cc} 
& $\begin{array}{c}\text { DAT } \\
(n=15)\end{array}$ & $\begin{array}{c}\text { Control } \\
(n=15\end{array}$ \\
\hline Male & 10 & 10 \\
Female & 5 & 5 \\
Age & $69.9(8)$ & $69.5(9)$ \\
Ediucation & $10.3(4)$ & $8.1(2)$
\end{tabular}

\section{Material:}

Stimuli were presented using the same apparatus as is described in Chapter 3 and 4. All words presented in the experiment were of intermediate frequency $(6 / 100.000<$ freq. $<90 / 100.000$; Uit den Boogaart, 1975). Words varied in length between three and four letters and were presented in upper-case letters. In the training session a list of 50 words was presented for perceptual identification. These words were not repeated in the test phase of the experiment. For the study phase of the experiment a list of 60 words was constructed. For the test phase three lists of words were generated. Each list consisted of 20 words which had been presented in the study phase of the experiment (Old words), and 20 words which served as fillers (New words). Each word from the study phase was repeated only once in one of the test lists. This resulted in three test lists of 40 stimulus presentations each. 


\section{Pracedure:}

First, the same base-line procedure as is described in Chapter 4 was given. This was followed by the study phase. All words were presented for study under disguise of a reaction time measurement, thus diverting attention away from any memory component of the study task to avoid the use of "involuntary" explicit memory strategies (see: Schacter, 1987). They were Instructed to read the words aloud as fast as possible and that their reaction times would be recorded. Reaction times were not actually recorded. No reference was made to an impending memory test. The experimenter pressed the button and the first word appeared on the screen. Hall a second after the onset of a verbal response was registered by the voice-key the word disappeared. This was repeated untill all 60 words had been presented for study.

Immedlately after completion of the study phase the first perceptual identification tasik of the test phase was given (delay $=0$ min). Fifteen minutes after completion of the study phase the second perceptual identification task was given (delay $=15 \mathrm{~min}$ ), and the third after 45 minutes (delay $=45 \mathrm{~min}$ ). Test lists were rotated across delays and subjects. Word presentation and feedback procedure (at second presentation only) were the same as described in Chapter 3.

\section{RESULTS}

The data of the perceptual identification tasks were analyzed with ANOVA in a $2 \times 3 \times$ 2 Group (DAT patients vs. normal controls), Delay (delay $=0,15$, and $45 \mathrm{~min}$ ), Facilitation (new vs. old words) design " with repeatted measures on the last two factors. In table 19 the results of the perceptual identification task are presented, in Figure 13 the decline of the facilitation effect as a function of increasing interval is illustrated.

There is no difference in base-line performance between groups on any of the three delays (delay $=0$ min: $F(1,28)=1.5$; delay $=15 \mathrm{~min}: F(1,28)=2.7$; delay $=45 \mathrm{~min}$ :" $F(1,28)=2.7)$ : the percentage correctly identified new words is similar at all delays. There is a main effect for delay, $F(2,56)=5.7, p<0.01$. A facilitation effect can be observed at all delays, $F(1,28)=51.5, p<0.01$. A decrease in the amount of facilitation over delays can be observed, $F(2,56)=3.4, p<0.05$. None af the other terms reaches significance.

There is no difference between groups in the decay of the facilitation effect over the three delays, $[F(1,28)=0.1]$. Nor do the groups differ in the rate of decline of the facilitation effect from the first to the second delay, $[F(1,28)=0.3]$, or for the decay in facillitation from the second to the third delay, $[F(1,28)=0.1]$. Thus the activation necessary for the manifestation of the facilitation effect does not decline at different rates for DAT patients and normal control subjects.

Stimulus presentation durations were nonparametrically analyzed by a Mann-Whitney $U$-test corrected for ties. DAT patients need more time to correctly identify $40 \%$ of the words, $z=4.2 ; p<0.01$ (DAT patients: mean $=74.3$ msec $_{2} S D=118.5$; Control subjects: mean $=23.7$ msec, $S D=8.8$ ).

The results found in the present study are in accordance with the results we found in our previous studies: Performance of the DAT patients is analogous to that of normall elderly control subjects. 
Table 19: Correctly Identified Words as a Function of the Interval between Study and Test (SEM) between Parentheses).

\begin{tabular}{l|cc} 
& DAT & Control \\
\hline O Minutes & & \\
New Words & $53.3(5)$ & $45.7(4)$ \\
Old words & $66.0(5)$ & $63.7(2)$ \\
15 Minutes & & \\
New Words & $51.0(5)$ & $39.0(5)$ \\
Old Words & $62.3(5)$ & $51.3(4)$ \\
45 Minutes & & \\
New Words & $51.3(6)$ & $39.0(5)$ \\
Old Words & $55.0(6)$ & $46.0(6)$
\end{tabular}

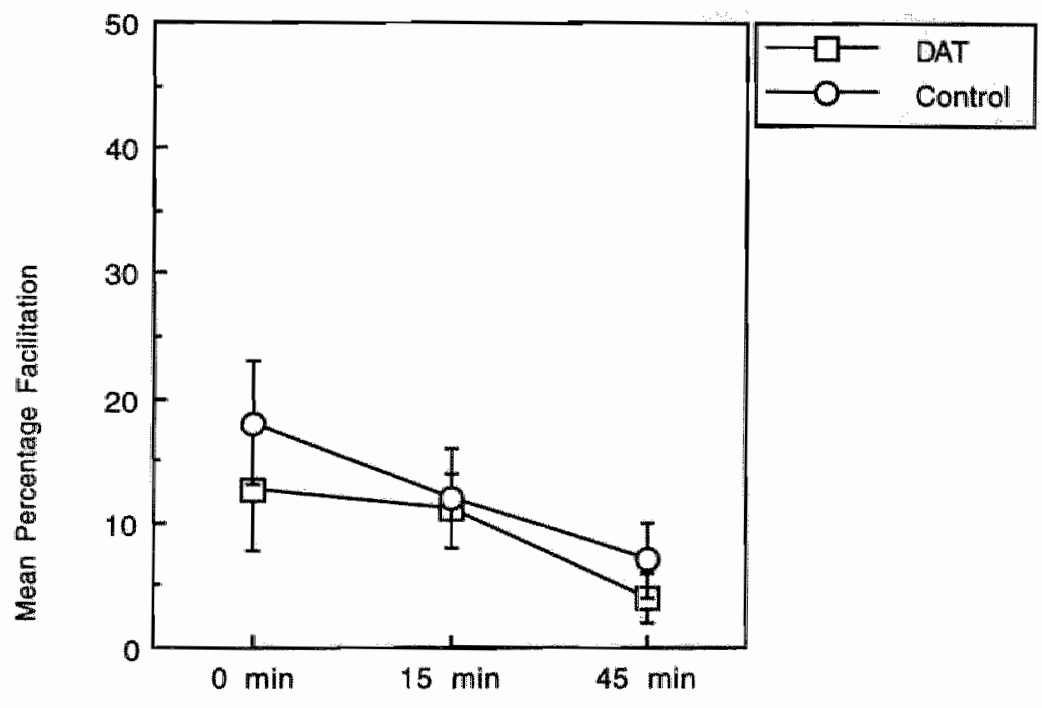

Figure 13: Mean Percentages Facilitation as a Function of the Interval Between Study and Test. 


\subsection{A COMPARISON OF THE PERFORMANCE OF YOUNG, MIDDLE- AGED, AND ELDERLY SUBJECTS ON PERCEPTUAL IDENTIFICATION AFTER THREE DIFFERENT DELAYS (EXPERIMENT 10)}

\section{METHOD}

\section{Experimental Subjects:}

Twenty-rine young, twenty-four middle-aged, and twenty-five elderly subjects were tested on perceptual identification performance after three varying delays. The selection procedure and criteria of experimental subjects were similar to those described in experiment 4 and 7 . Demographic data are presented in Table 20. Material and procedure were the same as described in the previous experiment.

Table 20: Demographic Data of the Three Age-Groups.

\begin{tabular}{l|ccc} 
& $\begin{array}{c}\text { Young } \\
(n=15)\end{array}$ & $\begin{array}{c}\text { Middle-aged } \\
(n=10)\end{array}$ & $\begin{array}{c}\text { Old } \\
(n=11)\end{array}$ \\
\hline Male & 15 & 14 & 11 \\
Female & 14 & 10 & 114 \\
Age & $21.8(4)$ & $55.2(8)$ & $74.8(4)$ \\
Education & $12.6(2)$ & $10.2(3)$ & $9.1(4)$
\end{tabular}

\section{RESULTS}

Data were analyzed in the same way as is described in the previous experiment. As can be seen in Table 21, there is a difference in base-line identification performance between the groups on delay $=0 \mathrm{~min}, F(2,75)=5.0 ; p<0.01$, which has disappeared on dellay $=$ $15 \mathrm{~min}[F(2,75)=2.6]$, and delay $=45 \min [F(2,75)=0.5]$.

There is a main effect of delay, $F(2,150)=14.6, p<0.01$. A facilitation effect can be observed at all delays, $F(1,75)=237.1, p<0.01$. Furthermore, the amount of facilitation decreases over delays (See: Figure 14), $F(2,150)=29.1, p<0.01$. And, most importantly, elderly subjects show less facilitation, $F(2,75)=6.9, p<0.01$. None of the other terms reaches significance.

Tukey's post-hoc analyses on the facilitation effects at each delay reveals that the elderly show less faclitation than the young group, and also less facilitation than the middle-aged group on the first and the second delay. At the third delay all groups exhibit an equal amount of facilitation.

Additional trend-analyses were performed on the facilitation effects. This was done to evaluate whether the decay of the facilitation effect over delays was different 
Table 21: Correctly Identified Words as a Function of the Interval between Study and Test (SEM between Parentheses)

\begin{tabular}{l|ccc} 
& Young & Middle-aged & Old \\
\hline O Minutes & & & \\
New Words & $33.1(3)$ & $42.3(3)$ & $44.4(3)$ \\
Oid words & $63.5(3)$ & $68.8(2)$ & $61.4(3)$ \\
15 Minutes & & & \\
New Words & $34.7(4)$ & $40.0(3)$ & $46.2(4)$ \\
Old Words & $54.3(4)$ & $56.3(3)$ & $54.2(3)$ \\
45 Minutes & & & \\
Now Words & $37.4(4)$ & $42.3(4)$ & $41.4(4)$ \\
Old Words & $46.2(4)$ & $51.0(4)$ & $48.4(3)$
\end{tabular}

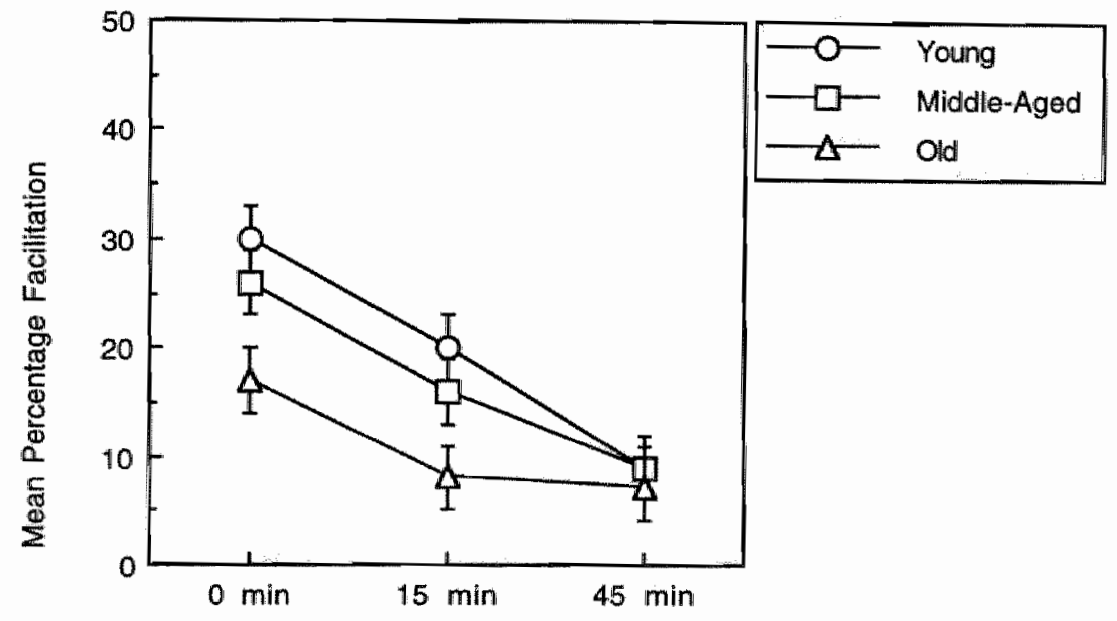

Figure 14: Mean Percentages Facilitation as a Function of the Interval Between Study and Test.

between groups. Therefore, the general means, linear and quadratic trend coefficients of the facilitation effects were calculated over the three delays. They were analyzed by a Group (Young, Middle-Aged, Old) by Delay $(0,15,45 \mathrm{~min}$ ) ANOVA with repeated measures on the last factor. Also, general means and linear trend coefficients were computed separately for the facilitation effects from the first to the second delay, and from second to the third delay. These were analyzed by a 3 by 2 (Group by Delay) ANOVA. 
Trend-analyses revealed the following: no differences between groups in the linear trend component over the three delays can be seen. $[F(2,75)=2.7]$. Also, the decay of the facilitation effect from the first to the second delay does not differ between groups $(F(2,75)=0.1]$, nor does it differ from the second to the third delay $[F(2,75)=1.9]$. Thus, the difference in facilitation effects between the elderly and the other two agegroups cannot be explained by faster decay rates.

Kruskal-Wallis one-way analysis of variance by ranks, corrected for ties of the stimulus presentation durations reveals a difference between groups: $H(d f=2)=36.02$, $p<0.01$. (Young: mean $=15.3, S D=5.0 ;$ Middle-aged: mean $=20.8, S D=5.7 ;$ Old: mean $=28.4, \mathrm{SD}=7.9$ ).

\subsection{DISCUSSION}

A similar pattern of performance can be observed for DAT patients and normal elderly on the present task. There is, however, an age-related deficit in perceptual identification performance on the interval task on the first and the second delay: Middle-aged subjects perform as good as the young subjects, but the elderly exhibit less facilitation than both the younger groups. However, the rate at which the facilitation effect declines is similar for all age-groups.

What can also be observed in all groups is that the facilitation effects in the present task decays rather steeply from the first to the second delay. Musen and Treisman (1990), for example, found hardly any decay in the repetition priming effect for linepatterns over a delay of 8 days. Sloman, Hayman, Ohta, Law, and Tulving (1988), on the other hand, found that the priming effect in a fragment-completion task showed a fast decline over the first 5 minutes. After 5 minutes, the decline in activation was very slow. The discrepancy in the rate of decline of the experiment of Sloman et al. (1988) and that of the study of Musen and Treisman (1990) is attributed by the latter authors attributed to specific characteristics of the stimuli. They state "[..] that the uniqueness, novelty, and specificity of the visual detals of the stimuli all contribute to priming of long duration (p. 135)". Their line-drawings are of course more unique and novel than words.

Our experiment in which words served as stimuli show similar results to that of the study of Sloman et al. (1988): Between a delay of 0 minutes and that of 15 minutes the rate of decay is considerable. But after a delay of 45 minutes one can still observe a facilitation effect. Although we did not test at intermediate nor at longer intervals. the facilitation at this point in time might have reached asymptote. The remaining facilitation effect at the 45 minute delay might be very persistent. From figure 14 it might be inferred that elderly subjects reach this asymptote earlier than the other agegroups because their initial activation level is lower, and not because facilitation declines more rapidly.

\section{Some Suggestions To Explain The Age-Differences.}

In the introduction it was suggested that the age-related deficit observed in the auditory feedback task was possibly caused by either a greater sensitivity to a change in modality of the elderly, or a greater sensitivity to limitations in stimulus duration at first presentation. First, we will further explore these alternatives. 
Sensitivity To Modality Changes: Episodic Processes.

As is explained before, episodic information is modality- and context-specific, while semantic information is modality- and context-independent.

In the Salasoo et al. (1985) model, facilitation only occurs if an episodic image is formed. This episodic image is based on the analysis of context codes, and strengthened by the results of the analysis at the letter (and feature) code level.

Craik and his associates (Craik, 1977, 1983, 1986, Craik \& Byrd, 1982; Craik \& Rabinowitz, 1984; Craik \& Simon, 1980) suggest that elderly subjects come to rely more on contextual information. If this is the case, one can assume that no age. difference will be observed if the task at hand provides sufficient contextual suppoit at retrieval.

In other words, if the episodic image formed at first presentation of a stimulus contains sufficient contextual cues that overlap with the contextual cues activated at second presentation, no age difference in implicit retrieval will be observed.

Assuming that older adiults rely more on context the probability will increase that an age difference will occur the less complete the overlap between study and test is. The overlap between study and test will be less whenever there are fewer contextual cues available or activated at either end (i.e. study and test).

As can be seen in figure 12, retrieval conditions are always the same in all procedures and for all subjects. The origin for differences in outcome concerning the facilitation effects must therefore be sought at the encoding end.

By presenting the stimulus in another modality fewer context cues which can improve visual retention are available, so this could explain the reduced performance of the elderly.

On the other hand, how can the results of the interval task be explained? Context cues are available to the same extent as in the visual feedback task and still an agerelated deficit arises. This could mean that the available context-cues are not analyzed to the same extent in older adults as in younger adults.

Sensitivity To Modality Changes: Semantic Processes.

Another possible mechanism to explain the age-difference found in the auditory feedback task might be based on the assumption that there is a deficit in semantic processing in the elderly. In general, crossmodal priming effects are presumed to result from activation of the semantic codes (Kirshner, Dunn \& Standen, 1989). According to this assumption, facilitation in the auditory feedback task is based upon semantic processing. According to the Salasoo et al. (1985) model, the activation of the semantic codes feeds back upon the letter and word feature level thereby strengthening the episodic images responsible for the facilitation effect. Thus, disturbances in semantic processes in the elderly would have an indirect impact upon the "completeness" of the episodic image. Although experimental support for agerelated deficits within the domain of semantic memory is scarce, Rabbit (1984) suggests that older adults have more difficulty in gaining access to semantic structures. Following his reasoning it can be argued that the more the task at hand requires access to semantic structures, the greater the age-related deficit will be. The presence of age-related impairments in the auditory and the interval task would thus mean that semantic processing plays a larger role in the facilitation effects of these tasks. 
Sensitivity To Stimulus Presentation Duration.

As is stated before, in the visual feedback procedure the stimulus is presented again until the subject has read the word aloud and thus a response is given. In other words; all processes in the Salasoo et al. (1985) model are completed. However, in the auditory feedback procedure no response is required from the subject at first presentation. Moreover, stimulus presentation duration is limited. Therefore, no inference can be made about how much processing has taken place when the first presentation is ended.

If elderly people are slower to process information they will be put at a disadvantage, compared to young people, the less time is given them to analyze stimuli. Suppose that the older subjects were only allowed time enough to analyze up to the letter-code level in the auditory feedback task. Young subjects, being faster to process information, would have completed the whole process to a greater extent. Consequently, the episodic image of the elderly group would be weaker or less complete. (Note that this may be caused either because access to semantic memory is only partially gained, or because analysis of contextual cues is less complete).

However, in contrast to what was expected the oldest age-group also showed less facilitation than the younger two groups at the first two intervals of the present task. Nevertheless, the facility to activate contextual cues at study was ample, and comparable to that of the visual feedback task. Therefore, a stimulus presentation duration long enough to build up episodic images is not the only, or predominant factor for the manifestation of an unimpaired facilitation effect in the elderly. If this were the case, unimpaired facilitation should have been found in the interval task, as it was found in the visual feedback task.

Some reflections on The Facilitation Effect of DAT Patients.

Whatever gross disturbances may be found in other memory tasks, no impairment can be discerned in the perceptual identification tasks used in the present study. In contrast to what was found concerning the amnesic Korsakoff patients in the study of Cermak et al. (1985), priming effect of DAT patients on immediate implicit retention in this task is not solely based upon semantic processing. Although DAT patients, as well as normal elderly, show less priming than younger subjects in the present study at delays of 0 and 15 minutes, facilitation can be observed at delays which exceed immediate recollection. Thus, it may be concluded that DAT patients, when compared to normal elderly, retain the capacity to form and use an episodic image. Also, the DAT patients in the present study do not display any evidence of a faster decline in the facilitation effect. Hence, these DAT patients do not exhibit an accelerated rate of forgetting, as measured by the amount of facilitation. The suggestion by Hart et al. (1987c), and Moss et al. (1986) that DAT patients forget at a faster rate cannot be confirmed by the present perceptual identification data. 


\title{
Chapter 6
}

\section{General discussion}

\author{
"What does the fish remind you of?" \\ "Other fish." \\ "And what do other fish remind you of?" \\ "Other fish." \\ Joseph Heller: Catch-22.
}

\subsection{SUMMARY OF THE RESULTS OF THE PRESENT STUDY}

The main objective at the outset of this study was to investigate whether tasks based on the perceptual identification paradigm could serve as a useful instrument to discriminate between the memory impairments of DAT and depressed patients at an early stage of the disease. The premise of this objective was based upon the assumption of Weingartner and his associates that DAT patients show deficits on tasks which are mainly based upon automatic processes, whereas depressed patients only show deficits on tasks that involve controlled processes. Impaired performance on automatic processing of DAT patients would also be different from the effects of old age as elderly subjects are presumed to be unimpaired on tests which are based upon automatic processes.

The performance of DAT patients on the perceptual identification tasks presented in this study is similar to that of their age-matched control groups. Only in exp. 4.1 do they differ from normal elderly in that they do not exhibit a facilitation effect on the auditory feedback task. However, in general, DAT does not result in deficits which are superimposed upon or qualitatively different from the effects of normal ageing on perceptual identification performance. Also, no difference can be observed between performance of DAT and depressed patients. The expected pattern of results, to wit a difference between normal elderly and depressed patients on the one hand, and DAT patients on the other, was not confirmed within the paradigm of perceptual 
identification. In view of these results, the main conclusion is that the perceptual identification paradigm cannol contribute to the development of a useful instrument for the early differential diagnosis between DAT and depression. However, the obtained data render some interesting questions in relation to the discussion of the effects of ageing and recent models of memory. These topics will be addressed in the next few sections.

The findings concerning the effects of ageing and perceptual identification performance can be summarized as follows: As is shown in Chapter 4, one short masked presentation of a stimulus does not result in a facilitation effect. The feedback provided in the perceptual identification tasks described in the first four chapters can be considered the first relevant presentation of stimuli. In other words, feedback can be viewed as the study presentation of items. As such, modality of presentation changes between study and test in the auditory feedback tasks, and is constant in the wisual reedback tasks. From the results of the auditory feedback tasks, it can be concluded that young and elderly subjects are differentially sensitive to changes in modality between study and test. Two possible explanations were proposed: the agerelated difference observed in the modality-changle condition (exp. 2 and exp. 8) results either from deficient semantic processing in the elderly, or from reduced processing of contextual cues which is considered to be an aspect of episodic processing (see Chapter 5).

The results of experiment 6 in which nonwords were used as stimuli show that elderly subjects are as capable as young subjects to form episodic images without the contribution of semantic processing under the proper experimental conditions. However the data obtained on the interval task suggest that the episodic images underlying the facilitation effect are not always as strong, or complete as they are in young adults. Even if ample time is given to analyze the words at study, older adults obviously do not always do so as efficiently as young subjects.

In the next few sections three central questions will be discussed. First, do tests based upon the perceptual identification paradigm serve as usefull instruments to discriminate between the memory performance of normal aged subjects, DAT patients, and depressed patients?

Second, how can the present results be interpreted in light of the episodic/semantic distinction? Topics, such as the relative contribution of episodic and semantic processes in perceptual identification, and the role of automatic and controlled processing will be discussed.

And last, how can the results presented in this thesis be related to what is generally found on tests of implicit memory, and could this have some theoretical implications?

\subsection{PERCEPTUAL IDENTIFICATION IN NORMAL AND MEMORY- IMPAIRED OLD ADULTS}

There is, to our knowledge, only one study (Light \& Singh, 1987) in which perceptual identification performance of young and elderly subjects was compared, and only few that assessed perceptual identification performance of DAT patients. Light and Singh (1987) tested young and older adults on two implicit memory measures, namely wordcompletion and perceptual identification, as well as on several explicit memory measures (cued recall, recognition and free recall). On all measures of explicit memory 
they found age differences, in contrast to what was found on the implicit memory measures. They did find a slightly poorer performance of the elderly on both implicit memory tasks, but these differences did not reach significance. They concluded that: The most conservative conclusion to be drawn [...] thus seems to be that there may be real, though small age effects in repetition priming and in word completion though probably not in perceptual identification (p. 539)". The assumption that perceptual identification performance may be unimpaired in the elderly is not corroborated by the outcomes of the present study. However, Light and Singh tested a group of elderly subjects with a mean age of below 70 . The age differences that arise in the experiments reported in this thesis only arise in subjects of over seventy years of age.

Grafman et al. (1990) investigated perceptual identification performance in DAT patients. They also tested a group of seven patients suffering from Progressive Supranuclear Palsy, but did not include a healthy, age-matched control group. They found only a marginal benefit from previous presentation of the stimuli in the DAT group. Besides the fact that no control subjects were tested, there is an additional problem with the procedure of the task they used. As in the present study, they did include a base-line procedure to individually assess presentation durations. However, the words that were to be repeated in the test phase of the experiment were presented for the first time in this base-line procedure. Hence, the base-line procedure also served as the study task. Unfortunately, no information is provided whether or not the words were identified at first presentation, nor about how long they had been presented. It is therefore quite possible, considering the extreme long presentation duratilions which have been found in the present study, that DAT patients did not percelve the words at all at first presentation. And, what has not been perceived at encoding at least up to a certain point, can not be facilitated at retrieval even if presentation duration is individually determined at the latter stage:

Keane, Gabrieli, Fennema, Growdon and Corkin (1991) tested DAT patients and healthy elderly in a series of experiments using perceptual Identification tasks. They briefly presented words, followed by a mask. If the word was incorrectly identified, it was repeated at a longer presentation duration (l.e. 16.7 msec longer). Their dependent measure was the time required to identify the stimulus. Although DAT patients needed disproportionally more time to identify the words, the pattern of priming effects was consistently comparable to that of the healthy elderly. These results are in accordance with the outcomes of the experiments described in the previous chapters.

In short, research concerning the relation between ageing and perceptual identification performance is still too limited to justify rellable conclusions. The research concerning perceptual identification performance in relation to the effects of DAT Is also limited. The few studies which did address this issue do not render unequivocal results.

\subsection{THE CONTRIBUTION OF SEMANTIC AND EPISODIC COMPONENTS IN PERCEPTUAL IDENTIFICATION}

To what extent episodic or semantic aspects contribute to various tests of memory is a much discussed issue. This is partly due to the vague definitions and constantly changing interpretations of the terms episodic/semantic. Some authors assume that 
only recall and recognition represent measures of episodic memory. In this case the episodic/semantic distinction is defined in terms of task variables (see, for a discussion of this issue McKoon et al, 1986). But other investigators define this distinction in terms of certain characteristics of the stimulus material. According to this view episodic information processing also plays a role in other tasks than recall and recognition, such as repetition or direct priming tasks. How much episodic or semantic aspects contribute in specific tasks is often hard to discern. For example, word stem completion is often considered to be a semantic lask. Generally, the presented fragments can be completed with a number of words. Completion by the previously studied word can be interpreted as a result of the semantic activation of the preexisting representation of that word. But even so, the fact that the stem is completed with that specific word may be a reflection of the episodic trace of the event being reactivated by the fragments. Jacoby and Dallas (1981) examined the relation between perceptual identification performance and recognition memory in a series of experiments. They found that some manipulations had similar effects on recognition memory and perceptuall identification, while others revealed different effects on the two tasks. For example, levels-of-processing manipulations (Exp; 1) influenced recognition memory, but had no effect on perceptual identification performance. Similarly, elaboration at encoding (Exp. 2a, b), or a longer study time (Exp. 3) increased the number of recognized items, but did not increase the number of identified words. Also, the facilitation effect observed in the perceptual identification suffered dramatically from a shift in modality (Exp. 6) whille it did not affect recognition memory. In contrast, number of repetitions at study, spacing of repetitions at study (Experiment $4 a, 4 b$ ) had similar effects on both tasks.

Jacoby and Dallas (1981) suggest that dissimilar effects of some factors are due to the different ways in which they influence the processing of the meaning of words (such as additional study time; see also: Stanovitch and West, 1983), while the factors that have similar effects on the two tasks exert their influence through the processing of graphemic and physical characteristics of an item. In other words, processing of meaning is irrelevant in perceptual identification tasks. Perceptual identification performance depends upon the processing of graphemic and physical characteristics. In line with this interpretation, Jacoby and Dallas noted that incorrect identifications had some physical similarities with the correct words but were not simillar in meaning. Thus, additional processing of meaning only enhances recognition performance. Semantic memory has only a limited, if any, contribution to perceptual identification according to Jacoby and Dallas (1981).

An interactive model is provided by Salasoo et al. (1985) described in the intraduction of this thesis. Briefly summarizing its major outlines, it is assumed that a fast identification response takes place in case of activation of existing codes, but that facilitation effects only arise if and when episodic images of the specific events are laid down to ald identification at second presentation. The analysis of contextual cues is the main source for the establishment of episodic images. The activation of existing word codes feeds back upon the letter and feature level. This feedback at the letter and feature level can enhance the formation of episodic images. Thus existing (pseudo)word codes can indirectly strengthen the results of the analysis of contextual cues. In case nonwords are presented, the existing codes which can strengthen episodic images are limited to the letter and feature level. However, if words are presented, pre-existing word codes (c.f. lexical access) provide additional support. The difference between the amount of facilitation of words and nonwords, which has been repeatedly demonstrated, either measured by an increase of the proportion correct identification 
of repeated items, or by a decrease in identification times at second presentation, is taken to reflect the influence of additional activation of premexisting word codes.

According to this model the relative contribution of semantic and episodic aspects can be manipulated. For example, by presenting nonwords (for which no representation in semantic memory is available), episodic aspects are the most important factor. In this case the episodic image for the nonword can only result from the analysis of context cues, plus an analysis of information up to the letter/feature level.

Semantic components, on the other hand, can be accentuated by changeing the modality between study and test. Cross-modall priming effects are presumed to result from activation of the semantic codes (Kirsner, Dunn \& Standen, 1989).

Generally, in studies on perceptual identification the facilitation effect of nonwords is smaller than that of words (Feustel et al., 1983; Schacter \& Graf, 1986a). Nornwords also take longer to be identified. The results of the perceptual identification task with nonwords presented in experiment 6 are in line with what has been previously reported. Moreover, this is the case for young persons as well as for old subjects. Also, increasing the number of intervening items (i.e. increasing lag) does not result in a decrease in the amount of facilitation, neither in the young, nor in the elderly subjects. Within the framework of Salasoo et al (1985), these findings suggest that processes necessary for the establishment of episodic images (that is, processing up to the level of letter and feature analysis (Cerella et al., 1982; Cerella, 1985; Hartley \& Mokenzie, 1991; Madden \& Allen, 1991), as well as the analysis of contextual cues) are intact in the elderly.

In summary, it is assumed that perceptual identification performance is neither exclusively episodically based, nor exclusively semantically based. It depends upon the task to what extent episodic or semantic memory (traces) are addressed.

\section{Episodic Memory in Normal and Memory-impaired Aged Subjects.}

As we have seen before, age-related deficits are most consistently found on recall and recognition tasks, considered to be the classical (explicit) tests of episodic information processing. Light (1990) states in her review on current views concerning age-related memory deficits that one of the few constant findings is a fallure of the elderly to process contextual information. Context-sensitivity is, per definition, one of the main characteristics of episodic information processing. However, Denney, Miller, Dew and Levav (1991) report quite different findings. They elegantly analyzed the relation between context and the episodic event (and its reflection: the episodic trace). They showed young and elderly subjects words printed on unique backgrounds. One group of subjects was instructed to remember the words, a second to remember the background, and a third to remember both. Subsequently, all subjects were tested for recognition memory of target information, context information, or both. By means of their design they were able to vary what represented target and what context information. In the words-only condition, words were target, and background was context. However, in the background-only condition, words were context and background was target information. Young subjects had higher recognition scores overall, but elderly did not show a specific impairment in recognizing cantextual information, whether contextual information was represented by the word, nor when context consisted of background information. These results suggest that elderly subjects are capable of analyzing context cues as well as young subjects. Craik (1983) suggests that old adults are able to analyze context cues, but onlly if the task or the data guide their attention in that direction. The results of the perceptual identification task with nonwords presented in this study render an explanation in terms of an age- 
related disturbance in episodic processing as such less probable.

Taken together, the abovementioned results suggest that older adults are capable of analyzing episodic aspects of tasks or stimulus material. However, they do not always do so spontaneously.

\section{Semantic Memory in Normal and Memory-impaired Aged Subjects.}

In general if is assumed that the basic semantic structures, as well as processes that are used to retrieve information from semantic memory are spared with age (Craik, 1977). For example, the majority of studies on spreading of activation within the semantic network suggests that there are no age-related changes in spreading of activation (Balota \& Duche $k_{,}$1988). These authors found that, although older adults exhibited an increase in lexical access time, activation within the semantic network builds up at the same rate in young and older adults. They even found somewhat larger semantic priming effects for the ellderly. They conclude that older adults are slower to input and output, but that there are no differences in the characteristics of activating related concepts in memory.

Another aspect of semantic memory was studied by Arbuckle, Vanderleck. Harsany \& Lapidus (1990). They investigated whether older adults show deficits in schema (c.f. general knowledge) availability or accessibility. They found a similar pattern of schema accessibility and avallability in young and old adults. Nebes et al. (1989) found no difference in semantic priming effects on a word naming task, nor on a lexical decision task between young and older adults, whereas Mitchell, Hunt and Schmitt (1986) found intact generation effects in the elderly.

The evidence for whether or not semantic memory is intact in DAT, is not unequivocal. Some studies report a breakdown of semantic knowledge (Chertkow \& Bub, 1990; Mitchell, Hunt \& Schmitt, 1986; Salmon, Shimamura, Butters \& Smith, 1988), some only find certain aspects of semantic memory to be impaired (Bird \& Luszcz, 1991; Herlitz \& Vitilanen, 1991), whereas others find preserved semantic processing. For example, Martin and Fedio (1983) investigated the organizational structure of semantic memory by requiring subjects to generate the names of items that could be found in a supermarket. DAT patients produced fewer categories, and fewer exemplars per category than normal controls (see also Ober, Dronkers, Koss, Delis \& Friedland, 1986). Nebes et al (1984) tested DAT patients on a semantic priming task to investigate whether the structure of semantic memory was intact. They presented words in a continuous list. The list was constructed so that every two words formed a pair, and the SOA was not specified. Subjects had to read aloud the words and reaction time of every second word in a pair was actually measured. Although the DAT patients were overall slower to react, their priming effect was similar to that of normal contrals.

Nebes et al. (1989) found a disproportional larger priming effect in their DAT patients compared to the priming effect in DAT patients they found in an earlier study (Nebes, Boller \& Holland, 1986). They postulated that, with incidental retrieval, the stimulus material and task situation themselves provide retrieval cues and guide memory access. They argued that, consequently, DAT patients should be most successful in tasks in which their access and use of semantic information is heavily constrained and guided by the task and stimuli.

The results of the studies described in the last two paragraphs indicate that if episodic memory is assessed with tasks that require little or no contribution from semantic processing, relatively limited, and inconsistent age effects are found. The same is true if the task at hand only relates to semantic memory processing. In the 
next section support for the view that age-related differences in performance are most obvious on tasks that involve a combined contribution of both semantic and episodic aspects will be discussed. Also, possible mechanisms to explain the age-related differences in light of the Salasoo et al. (1985) model will be discussed, as well as a more recent model of perceptual identification proposed by Masson \& MacLeod $(1992)$.

\section{Combining Semantic and Episodic Aspects to Explain Normal and Pathological Performance on the Perceptual Identification Tasks.}

Rabbit (1984) suggests that elderly people have a deficit in semantic encoding which results in impairments on episodic tasks in which semantic processing also plays a role (See also: Burke, White \& Diaz, 1987). Simillarly, Balota and Duchek (1988), and Anderson (1983) suggest that older adults produce large deficits in episodic memory tasks that demand semantic processing at both encoding and retrieval.

A similar suggestion concerning the deficits shown by DAT patients is offered by Randolph (1991). He states that episodic and semantic memory decline in parallel in DAT. Therefore, major deficits arise on tests that place a demand an both kinds of processing. Weingartner et al. (1983) also suggest that DAT patients, like normal elderly, seem to be deficient in using semantic information to facllitate their episodic memory. They presume that an impairment in gaining access to semantic memory might account for the major deficits DAT patients show on episodic memory tasks, although their data do not seem to justify such an assumption. However, supporting data for this notion are provided by Corkin (1982). She found that DAT patients did mot show the normal advantage of semantic processing to aid episodic memory (i.e. recognition memory).

The model of Salasoo et al. (1985) does not explicitly include the analysis of meaning as being part of the processing necessary for perceptual identification. Semantic aspects only play an indirect role in the establishment of episodic images in that existing word codes feedback upon the letter and feature level. Activation of these codes mainly serves to enhance rapid identification of items.

Masson and MacLeod (1992) present an interesting model in which they ascribe a specific function to the analysis of the meaning of words in perceptual identification. It is not contradictory to what Salasoo et al. (1985) presented. Moreover, the analysis of meaning and its function could easily be integrated within this model. Within this model, it is imaginable how semantic aspects can play a more important role in the establishment of facilitation effects. $\| n$ other words, they hypothesize a greater contribution of semantic memory on this episodic memory task, and describe a possible mechanism through which semantic aspects can contribute to episodic images. In a series of experiments Masson and Macleod set out to investigate the role of context in perceptual identification performance. Their main assumption is that the amount of priming is determined by to what extent operations acting at first presentation of a stimulus overlap with the operations acting at second presentation. A major difference with the model of Salasoo et al. (1985) described previously is that Masson and MacLeod"s model it is not the episodic Image that underlies the memory of an event, but the pattern of operations in itself forms the memory of the event. They refer to research presented by McClelland and Rumelhart (1981) in which visual presentation results in activation of candidates which are similar in features (e.g. heard - heart), while by auditory presentation a different set is activated (e.g. heard - hurt). Eventually the same meaning will be processed, but the discriminative operations to reach this end are different so that the overlap in operations to form an interpretation 
of the stimulus will be less if modality between first and second presentation is changed than is the case il modality is held constant.

According to Masson and MacLeod, there are two classes of encoding operations: Those contributing to the initial interpretation of a stimulus, which are highly contextspecific, and those elaborating on that interpretation (sometimes correcting inconsistencies of the initial interpretation). In perceptual identification the first class operates, in recognition both operate, but predominantly the elaborative operations. Interpretative operations are not only highly context-specific but also depend upon the recruittment of knowledge closely related to the meaning of stimuli. Characteristically , by presenting a stimulus a set of possible candidates is recruited and further processing results in discriminating the target from this set of alternatives and thereby suppressing or deactivating the knowledge that is acquired of the alternatives. These discriminative operations are due to processing of the meaning of the word. However, they demonstrated that the context in which a word is presented can determine whether or how an item is discriminated from its alternatives. For example, if a word is highly integrated in its context, as is the case with words embedded in a sentence, discrimination of the individual words does not take place (c.f. Oliphant, 1983), and little or no facilitation is observed when the isolated word is presented for perceptual identification. In perceptual identification of single words these discriminative operations are mecessary. Similarly, if a cue is presented from which an antonym is to be generated, later perceptual identification of the isolated antonym does not result in a facilitation effect. The explanation they give for this phenomenon is that by presenting the cue a set of possible candidates is generated which depends upon the meaning of the cue, while at perceptual identification the set of alternatives is determined by the meaning of the target (i.e. the antonym). Therefore, discriminative operations are different at first and second presentation.

In short, in this model semantic memory is hypothesized to contribute in perceptual identification performance in that analysis of the meaning of words is necessary to discriminate between alternatives whilch are being activated. Moreover, it offers an explanation for the reduction in facilitation by changing modality between first and second presentation. There is some facilitation because, eventually, the meaning of the word is analyzed. But the amount of facilitation is reduced compared to within-modality facilitation effects, because the overlap in operations between study and test is limited. That is, the difference in operations is a result of different routes to the construction of meaning of words.

The finding that there is an age-related difference between performance on the auditory feedback tasks may be viewed within the Masson and MacLeod model. Possibly, elderly subjects are particularly dependent on the amount of overlap between study and test (see also craik, 1983). In the auditory feedback tasks the route through which the meaning of words is reached at study (auditory route) is different from that by which the meaning of words is reached at test (visual route). So the overlap between study and test is less than in the perceptual identification tasks in which modality between study and test is constant. If memory performance of older adults depends more upon the amount of overlap between study and test, this could serve as an explanation for the results obtained on the auditory feedback tasks. However, a reduced amount of overlap between operations acting at study and test does not suffice to explain the results of the interval tasks. We therefore also have to assume that elderly people are less inclined to engage in self-initiated discriminative operations concerning the meaning of words.

The outcome of a study by McDowd and Oseas-Kreger (1991) provides support foi 
this latter notion. They investigated the relation between ageing and inhibitory processes in a negative priming experiment. Negative priming is based upon the active inhibition of irrelevant information. Core of this kind of priming is that if a stimulus which had to be ignored or inhibited (e.g. If It was a distractor to another stimulus) at first presentation is later presented as target information, processing of that stimulus will be impaired. The reason for this impaired processing is that operations at first presentation are directed at inhibition, whereas inhibiting operations are not appropriate at second presentation. Elderly subjects did not show such a negative priming effect, which led McDowd and Oseas-Kreger to the conclusion that inhibitory processes are impaired in the elderly. The results of a series of experiments of Rabinowitz and Ackerman (1982) also brace the assumption that elderly lack discriminating operations. They found that elderly people encode global semantic characteristics of stimuli, not enough to encode the unique features of the experimental context.

Further support for this assumption is offered by Masson and MacLeod in an explanation of the results of a study by Begg and Snider (1987). The latter investigated the effect of generating words versus the effect of just reading the words on cued recall. They only found an advantage on cued recall of generated words over words that had been read if the two encoding conditions were intermixed in the study task. They explained this outcome by postulating that the generate condition turned their subjects into "lazy readers". Masson and MacLeod extend this by arguing that subjects engaged in less recruitment of knowledge regarding the meaning of items necessary for perceptual identification. The same reasoning could apply to the outcomes of the interval task. If we assume that elderly people are less inclined to engage in discriminative operations to procure knowledge of the meaning of words, just reading the words, as is the case in the interval tasks, does not direct them to those operations.

Why, then, do elderly people not show a deficit in the visual feedback task? At first sight, there seems to be no difference between the first presentations of stimuli in the visual feedback tasks and the interval tasks. The stimuli are presented for similar presentation durations and also within the same modality. Notwithstanding the similarities, an age-related difference in performance is observed. However, an important difference is the measure of attention directed to the stimuli at first presentation. In the visual feedback task, an item can only be facilitated if it was incorrectly identified at first presentation. Thus, attention in the feedback procedure is drawn to the correct word. In the interval task, no specific attention is drawn to the individual items. The difference in the measure of attention at encoding obviously does not affect performance of young adults. Their facilitation effects are similar, whother facilltation is measured in the visual feedback tasks, or in the interval tasks. However, performance of the elderly does seem to be affected. Possibly, feedback which results in attention being directed to the correct stimulus (within the same modallty) induces the necessary discriminative operations in the elderly. Discriminating operations are irrelevant on the task in which nonwords are used. Nonwords present a unique bit of information and hence do not have to be discriminated from possible candidates, not at the time they are initially presented, nor at repeated presentation. Therefore. preserved priming for nonwords can be expected. As in the present study, the priming effect of nonwords is usually found to be smaller than that of words in young normal subjects (Feustel et al., 1983; Schacter \& Graf, 1986a). The difference in priming between words and nonwords could be taken as the contribution of semantic memory processing in the priming performance of normal subjects. 
An Age-Felated Impairment in Automatic or Controlled Processing?

Jacoby and Dallas (1981) state that the facilitation effect in perceptual identification is based upon the automatic retrieval of an episodic trace established at first presentation of stimuli. The conclusion of their series of experiments that even episodic information can be retrieved automatically, is not contradicted by the present results. However, although the way in which retrieval is tested is the same in all experimental conditions, performance of the elderly seems to be affected by the different encoding conditions of the tasks, or the differences in overlap between study and test. Kausler \& Hakami (1983) argue that automaticity at retrieval does not necessarily imply automaticity at encoding. Perhaps automaticity even pertains to the encoding phase of the perceptual identification, at least in some instances and probably only in the young. Perhaps elderly people fail to encode certain aspects automatically, whilst young subjects do. Hasher and Zacks' (1979) model concerns automatic processes at encoding. They proposed two types of automatic processes: inherited and practised. The first category entalls processes evaluating the frequency-of-occurrence, the spatial location, and the time of events. The second category consists of processes that act upon the evaluation of the meaning of words.

Kausler and his assoclates investigated in a series of experiments whether processes of the first category which Hasher and Zacks assumed to be automatic were unaffected by ageing. They did find age-dependency of processes which were presumed to be automatic. Moscovitch (1982) registered the reading-speed of old and new sentences. He found that young adults benefited more than old adults from having read the sentences before. From these findings it may be concluded that either the criterion of age-independence is not valid, or older adults show impairments in automatic processing. Light and Singh (1987) argue that the intention to remember is an important factor in determining the magnitude of age-differences. According to these authors, age-similarity is obtained when instructions direct attention away from conscious efforts to remember. In their study the facilitation effect is not reliably affected by age or encoding condition (pleasantness rating vs. vowel counting).

As suggested above, possibly young subjects analyze items up to a certain level, regardless of experimental condition in the modality-constant conditions (i.e. the visual feedback tasks and the interval tasks). Within the Masson and Macleod model, this would mean that whether or not attention is directed at identification of a stimulus the first time it is presented, young subjects engage in discriminating operations in order to recruit knowledge about the meaning of words automatically. Older adults do not seem to do so automatically. Instead, they need a certain amount of attention to use the meaning of words as a discriminating stimulus.

\subsection{IMPLICIT MEMORY AS A DESCRIPTION OF STRUCTURES OR TASK MANIPULATIONS}

In general, preserved implicit memory is found in aged subjects, at least on priming of single items (Hess \& Arnould, 1986; Kaus!er, Wright \& Hakami, 1981; Light, Singh \& Capps, 1986; Mitchell, 1989; Moscovitch, 1982). Our results on the older age groups, however, as well as recent results of other studies (Chiarello \& Hoyer, 1988; Hultsch, Masson \& Small, 1991; Mitchell, Brown \& Murphy, 1990; Rose, Yesavage, Hill \& Bower, 1986), pose some questions about the assumption that implicit memory is preserved in 
the elderly. For example, Davis et al. (1990) tested seven age groups on a word-stem completion task. They found impaired priming performance, but only in the older groups. Our results are in accordance with their results. We too do not find any reliable age differences until the age of 70 . However, Chiarello \& Hoyer (1988) even found impailred word-stem completion in samples under the age of seventy.

Considering the contradictory results obtained in the various studies on preserved implicit memory in the elderly it is difficult to hold a position that implicit memory is a unitary concept. Although different procedures are likely to pose a problem for the comparability of the various studies, even in the present study in which the general procedure is the same, and retrieval conditions are held constant between the varlous experiments, we see dissociable effects of ageing.

Therefore, there may be some questions about the validity and even the usefulness of making a distinction between implicit and explicit memory (See for a description of the theoretical pitfalls of this distinction. Richardson-Klavehn \& Bjork, 1988). The same methods that are used to demonstrate a dissociation between implicit and expllcit memory have recently been used to confirm a dissociation between various measures of implicit memory. For example, sometimes a dissoclation in performance can be abserved within the same patient group on several implicit memory tasks. Heindell, Salmon, Shults, Walicke and Butters (1989) tested a variety of patient groups with their proper controls on two implicit memory tasks, namely pursult-rotor motor learning and lexical priming in a word-stem completion task. They even found a double dissociation between their patient groups and the two tasks involved: DAT patients performed like their controls on the pursuit-rotor task but not on the word stem completion task. However, patients suffering from Huntington's disease showed exactly the opposite pattern. Bondi and Kaszniak (1991) compared non-demented patients suffering from Parkinson's disease with DAT patients on various measures of implicit memory (Exp. 1: skill-learning and perceptual memory in a perceptual identification task with pictures, Exp. 2: word-stem completion, Exp. 3: pursuit rotor tracking (motor-skill learning), and explicit memory (Exp. 1: free-recall, Exp. 2: cued recall). The DAT patients were impaired compared to normal subjects and Parkinson patients on perceptual memory, and word-stem completion, but not on skill-learning, or pursuit rotor tracking (considered to be procedural memory tasks). Keane et al. (1991), however, also found a dissociation in DAT patients between word completion and perceptual identification. Their patients were impaired on word-completion, but not on perceptual identification. The difference in outcome between the former and the latter study, might be attributed to different kind of stimull that were used (pictures vs. words).

Even stronger evidence comes from a study of Perruchet \& Baveux (1989). They analyzed data of four implicit memory tasks by means of correlation and factor analysis. They tound a relation between performance on a perceptual clarification procedure and a word completion test on the one hand, and a relation between tachistoscopic identification and anagram solution on the other hand. The latter two tests, however, were not related to the first two tests. They state that implicit memory can be affected by the nature of the initial encoding or by orientation of attention during initial exposure to stimulation. Hunt \& Toth (1990) also found a dissimilar effect of orthography on word fragment completion and perceptual identification. Orthography influenced word fragment completion but did not affect perceptual identification performance.

And finally, Witherspoon and Moscovitch (1989) found stochastic independence between two implicit memory tasks. Word fragment completion and perceptual 
Identification were independent from one another, although the order in which these tests were given determined whether independence was found (word stem completion before perceptual identification rendered dependency, probably because subjects became aware of a relationship between the two tasks and performance might have been influenced by strategic processing. When the overlap between the two tasks (exp: 2) was reduced, again no dependency was found). In short, the evidence against the existence of implicit and explicit memory as two separate memory systems is accumulating.

Even if the distinction is used in a descriptive way, that is, to describe types of memory tasks, it does not seem very useful. As stated before, implicit and explicit memory, by definition, only relate to the retrieval end of memory processing. In our experiments the conditions under which implicit retrieval took place were always the same: First, subjects were not informed about the nature of the memory task and not even instructed to remember the material (reducing the possibility of "involuntary explicit memory ${ }^{\mathrm{j}}$ ). Second, the retrieval of the previously presented information was measured by shortly presenting the stimuli for a second time. Thus, retrieval was always the same, whether the task at hand involved auditory, visual or no feedback at all, or whether the second presentation was given after a certain interval. In spite of the retrieval conditions being the same, differential results concerning age-related impairments were observed. Hence, even within the same experimental task, no constant preserved implicit memory could be observed. What did, however change, were the conditions under which encoding took place. Perruchet and Baveux (1989) also state that implicit memory can be affected by the nature of the initial encoding task or by orientation of attention during initial exposure to stimulation (see also the results of Forster \& Davis, 1984 and Oliphant, 1983). Therefore, it may be more prudent to use categories like the intentional/incidental distinction just as a description of what kind of instructions were given, not only at the encoding end, but also at the retrieval end of information processing.

Instead of plunging into some theoretical labyrinth it is perhaps better to take task variables at both encoding and retrieval (and the amount of overlap) into consideration to map the memory impairments accompanying old age (See also: Light, 1991; Perruchet \& Baveux, 1989; Witherspoon \& Moscovitch, 1989). 


\section{Chapter 7}

\section{Summary and conclusions}

"They also depend upon the a priori images we have accumulated in our memory. We constantly seek to find [..] analogues to our previous experiences. If we didn't we"d be unable to act".

Robert M. Pirsig: Zen and the Art of Motorcycle Maintenance.

\section{INTRODUCTION}

The original purpose of the present study was to explore whether it would be possible to discriminate between the memory impairments of depressed patients and patients suffering from Dementia of the Alzheimer Type using memory tasks which are based on paradigms from experimental psychology. Especially at an early stage of DAT, differential diagnosis can be very complex. On the one hand, DAT patients may be well aware of their impairments, and as a result may develop a depressed mood state. On the other hand, depression, especially in the elderly, may be accompanied by memory impairments.

in Chapter 1 an effort is made to survey the current views on the memory impairments which occur as a result of the normal ageing process, depression in old age, and Dementia of the Alzheimer Type. These are particularly discussed in relation to three theoretical frameworks from experimental psychology: the distinction between implicit and explicit memory, the distinction between automatic and controlled memory processes, as well as the distinction between episodic and semantic memory.

The results of the majority of studies on the memory performance of DAT patients show that almost all aspects of memory are affected by the disease. Concerning the memory impairments of depressed patients it is generally assumed that impairments occur as a result of factors not directly related to memory, such as changes in motivation or changes in the use of strategies. Such factors play a crucial role on tasks which are based upon the so called controlled memory processes. Controlled processes require conscious attention, can be enhanced by practice, and are negatively 
affected by fatigue and ageing. The quality of controlled processing is related to intelligence. In contrast, automatic processes cannol be strengthened by practice, are independent of educational level. and the use of strategies. Once started, they cannot be interrupted. Per definition, age is of no significance to this kind of processes. It is generally assumed that the so called priming tasks are based upon automatic processes.

A priming task was used in the experiments described in this thesis: the perceptual identification task. Words are presented on a screen for a very short duration. In the base-line procedure the presentation duration is assessed for each individual so that the chance to correctly identify the words is about $40 \%$. As such, the percentage correct identifications at first presentation of the words is the same for all individuals.

Words are repeated in the test procedure of the experiments. Repetition of the words results in a facilitation effect: the difference in percentage correct identification between the second and the first presentation of the words. This facilitation effect is assumed to reflect the automatic retrieval of an episode (i.e. an event tied to its temporall and spatial context).

DAT patlents and depressed patients were selected by and tested in a hospital in Helmond (the Netherlands); this hospital has changed names three times during the course of the study (from "het Lambertus Ziekenhuis", to "het Streekziekenhuis Helmond/Deurne", to "het Elkerliek Zlekenhuis"). Most of he control groups of normall elderly were community-dwelling elderly and tested at the Department of Neuropsychology and Psychobiology of the University of Limburg (Maastricht).

\section{Experiment 1}

In the first experiment performance of a group of moderately to severely impaired DAT patients was compared to that of a group of normal elderly (matched for age and education) on a perceptual identification task and a recognition task.

Automatic processes are, by definition, unaffected by ageing. In contrast, DAT is assumed to afflict nearly all aspects of memory, among which the automatic processes. Therefore, it was hypothesized that DAT patients would exhibit less facilitation on the perceptual identification task. If words were incorrectly identified auditory feedback was given in this experiment.

In contrast to what was hypothesized. DAT patients showed a similar amount of facilitation as the normal elderly group. However, the facilitation effect was smaller than expected in both groups. The groups did differ in their performance of the recognition task.

\section{Experiment 2}

To examine whether the assumption of age-independence of automatic processing also applies to the processes underlying the facilitation effect an additional experiment was done. The performance of a group young healthy subjects was compared to that a group of old subjects on the same perceptual identification task. If automatic processing is not affected by ageing both groups should exhibit similar amounts of facilitation. This was not the case: The elderly group showed less facilitation than the young group.

\section{Experiment 3}

In a third experiment, parallel to the experiments described above, a group of imild to moderately impaired DAT patients, as well as a group of depressed patients were 
tested on the same perceptual identification task. Performance of both patient groups was compared to that of a control group. The latter consisted of patients who visited the hospital because of memory complaints, which could, however, not be objectifled by extensive neuropsychological testing. Again, no difference existed between the facilitation effects of the DAT patients and the control group. Similarly, no difference in facilitation performance arose between the two groups of patients. Concerning the results of the recognition task there was a marginal difference between DAT and depressed patients on the one hand and the control group on the other hand. The results of the first three experiments were contrary to what was expected:

1. DAT patients showed a similar amount of facilitation as the depressed patients and the normal elderly.

2. The facilitation effect resulting from this experimental procedure did seem to be age-dependent.

Based on the results of experiment 2, the general objective of this study was extended to the effects of normal ageing on the perceptual identification task. Therefore, paired experiments are described in the subsequent chapters, of which one involves the comparison of groups of patients and a group of control subjects, and the other concerns the comparison of different age groups of normal subjects.

\section{Experiment 4}

As was stated before, the amount of facilitation in the previous experiments was rather smaller than expected. Possibly an explanation is to be found in the assessment of the base-line presentation duration. Due to the limitations of the computer screen which was used, the presentation duration could only be increased by intervals of 20 msec. Perhaps the performance of the separate experimental groups is differentially influenced by such a step-wise increase. Second, it is known that changes in modality of presentation between first and second presentation diminish the facilitation effect. And third, the size of the words was rather small, which could be more disadvantageous for the elderiy groups.

Conceivably, a difference in the facilitation effects between experimental groups was lost in a floor-effect. The procedure and apparatus were modified to circumvent the abovementioned impediments. A screen consisting of LED-displays was developed. With this screen presentation duration could be varled with intervals of $5 \mathrm{msec}$. Also the size of the words was considerably increased. The procedure was adapted in that visual feedback was given after a word was incorrectly identified.

Mildly to moderately severely impaired DAT patients were compared to depressed patients and normal control subjects. The facilitation effect resulting from the modifled procedure was more substantial than in the previous experiments. However, again, no general difference between the patient groups on the one hand and the control group on the other hand could be demonstrated. There only was a difference between DAT patients and depressed patients on the facilitation of low-frequency words (however, depressed patients and the control subjects did not differ).

\section{Experiment 5}

To explore if an age-related decrease in the facilitation effect could be demonstrated with the modified procedure, six age groups were tested. Subjects varied in age between 20 and 80 . Although there was a slight reduction in the facilitation effect with increasing age, no difference existed between the age groups. 


\section{Experiment 6}

In this experiment performance on a perceptual identification task in which the stimulus material consisted of nonwords was compared to that on a perceptual identification task with regular words. Three age groups were tested.

In general it is assumed that the most widespread memory impairments arise as a result deficient episodic memory processing. In contrast to existing words, nonwords are not represented in semantic memory. The hypothesis was that if the difference in facilitation observed in the tasks in which auditory feedback was given resulted from impairments in episodic memory processing then again an age-related reduction in the facilitation effect should arise on the task in which nonwords were presented. However, this hypothesis could not be confirmed: The three experimental groups exhibit similar facilitation effects on the nonword task.

Summarizing the results of the previously presented experiments:

- An age-related reduction of the facilitation effect can be observed if auditory feedback is provided.

- No age-related impairment of the facilitation effect can be demonstrated if visual feedback is given.

- The age-related impairment observed in the auditory feedback task does not seem to result from deficient episodic processing.

- DAT patients generally do not differ from depressed patients, nor do both patient groups differ from control subjects in perceptual identification performance.

\section{Experiment 7}

One of the adaptations in the perceptual identification task used in the experiments described in the previous chapter was a change in feedback procedure. Other modifications included changes in base-line presentation duration assessment and the use of a different screen. Also, differences in experimental groups could be held responsible for the differences in facilitation performance found between the experiments described in Chapter 2 and those described in Chapter 3 . Furthermore, the role of feedback in the manifestation of the facilitation effect was not clear.

Therefore, type of feedback procedure was varied as a within-subject factor. A group of DAT patients and a group of normal controls were tested on three different perceptual identification tasks: one with visual feedback, one with auditory feedback, and another without feedback. Thus, an attempt was made to replicate the results of the auditory feedback task, and directly compare with the results on the visual feedback task with the factors apparatus and base-line assessment held constant.

Based upon the results of the previous experiments it was hypothesized that the experimental groups would exhibit similar amounts of facilitation on all tasks. This was confirmed by the results. However, an unexpected outcome was that no facilitation effect ensued from the first presentation of a word if no feedback was given after an incorreot identification.

\section{Experiment 8}

Three age groups were tested on the three feedback tasks. If the age-related reduction of the facilitation effect on the auditory feedback tasks described in the second chapter were to be understood as the result of artefacts due to apparatus or procedure then no such age-related effects were to expected in this experiment. However, the results did correspond to what was found earlier: There was an age- 
related decrease in the amount of facilitation on the perceptual identification task in which auditory feedback was given, but not on the task in which visual feedback was given. And again, no facilitation effect could be observed if no leedback was given.

Summarizing the results of experiment 7 and 8 :

- An age-related difference exists in the facilitation effect on the auditory feedback task, which cannot be explained by artefacts due to variations in apparatus or procedure...

- Such an age-related difference does not exist if visual feedback is given in the otherwise similar task.

- No facilitation effect ensues from the first presentation of a word if no feedback is given.

- DAT patients do not differ from normal elderly in their performance on any of these tasks.

\section{Experiment 9}

From the aforementioned it may be concluded that facilltation only arises if feedback is given. Thus, feedback may be considered as the first (relevant) presentation of a stimulus. The differential results on the two (visuallauditory) feedback tasks must be the result of the differences between the two tasks. The first difference is modalitychange vs. modality constancy between first and second presentation. The second difference is the presentation duration of the first (note: feedback) presentation. In the auditory feedback task the stimuli are presented for as long as it takes to pronounce a word. In the visual feedback task, the word only disappears from the screen after subject have had ample time to analyze and pronounce the word. Possibly, elderly subjects are more sensitive to changes in modality, or to limited presentation duration. Therefore, a new perceptual identification task was developed. In this task, first presentation of the words was comparable to that of the visual feedback task in that arnple time was provided to analyze and pronounce the words, and words were presented within the visual modality. After three different delays a perceptual identification task was given. In this way the course of the facilitation effect over time could be explored.

A group of DAT patients was compared to a group of normal contral subjects on the interval task. Finding no differences on the perceptual identification tasks described in the previous chapters, no difference between groups was expected, and this hypothesis was confirmed by the results.

\section{Experiment 10}

Young, middle-aged, and old subjects were subjected to the interval task. It was hypothesized that if the age-related differences found until now were due to a differential sensitivity of the elderly to changes in modality or presentation duration then no age-related impairment should arise on this task in which these factors were similar to that of the visual feedback task.

However, the results show a difference in the facilitation effect on the first and second delay (1.e. 0 and $15 \mathrm{~min}$., respectively) between the oldest group on the one hand and the two younger groups on the other hand. Also, the facilitation effect in all groups decays rather steeply from the first to the second delay.

The results are discussed in light of the distinction between episodic and semantic processes. Also, the effects of possible differential sensitivity to variations in stimulus presentation duration of the elderly are discussed. 


\section{Chapter 6}

In this chapter the results of the present study are discussed within the different frameworks. First, the outcomes are related to the distinction between episodic and semantic memory. The relative contribution of both episodic and semantic processes to the manifestation of the facilitation effect is evaluated.

It is concluded that the relative contribution of either type (semantic or episodic) of processes depends upon the specific task characteristics.

Subsequently, the results on the effects of normal and pathological ageing of the present study are discussed within the episodic/semantic framework. They are related to the model of Salasoo et al. (1985), and a more recent proposition of Masson and Macleod (1992), in which a feasible interactional process between contextual aspects and aspects of meaning is proposed. A tentative explanation of the present results could be that elderly subjects are less inclined to engage in self-initiated discriminative operations concerning the meaning of words.

Next, the results are evaluated within the framework of automatic/controlled processing.

It is concluded that the criterion of age-independence of automatic processes needs to be reconsidered. The results of the present study, as well as those of several other studies, show that either automatic processes not always prove to be unaffected by the consequences of ageing. Or, it must be conciuded that processes which up until recently were assumed to be automatic cannot be labelled as such.

The discussion concerning the distinction between implicit and explicit memory, leads up to the recommendation to scrutinize task varlables more closely instead of adding new paradigms which only obscure the discussion in the vast domain of research on memory.

It is concluded that implicit memory, whichever interpretation is given to the term, cannot bo viewed as a unified concept.

The general conclusions of the study presented in this thesis can be summarized as follows:

- DAT patients do not differ in the amount of facilitation on the perceptual identification tasks, used in this study, neither from depressed subjects, nor from normal control subjects. Thus, these perceptual identification tasks do not provide the basis for a possible instrument to serve the purpose of differential diagnosis at an early stage of the disease.

- The criterion of age-independence of automatic processes , proposed by Hasher and Zacks (1979) is untenable, and should be reconsidered.

- The systematic variation of task characteristics sheds a different light upon the usefulmess to distinguish between implicit and explicit memory.

- The perceptual identification paradigm offers an interesting and promising instrument in the exploration of the basic mechanisms underlying impaired memory performance in the elderly. 
De oorspronkelijke opzet van dit onderzoek was te onderzoeken of met behulp van experimenteel-psychologische taken de geheugenstoornissen van patiënten, lijclend aan Dementie van het Alzheimer Type (DAT) te onderscheiden zouden zijn van de geheugenstoornissen die depressieve patiënten vertonen. Vooral in een vroegtijdig stadium van DAT is dit onderscheid moeillik te maken, omdat enerzijds, een patiënt in het beginstadium van DAT zich zeer wel bewust kan zijn van de hiaten in zijn functioneren, en als gevolg daarvan een depressieve stemming kan ontwikkelen. En, anderzijds, kan een depressief toestandsbeeld gepaard gaan met geheugenstoornissen.

In hoofdstuk 1 wordt getracht een overzicht te geven van de vigerende opvattingen met betrekking tot de geheugenstoornissen die gepaard gaan met depressie, DAT en het normale verouderingsproces. Deze worden in het bijzonder besproken in het licht van drie theoretische kaders: het onderscheid tussen impliciet en expliciet geheugen, het onderscheid tussen automatische en gecontroleerde geheugenprocessen en het onderscheid tussen episodisch en semantisch geheugen.

De resultaten van onderzoek naar de geheugenstoornissen van DAT patiënten tonen aan dat nagenoeg alle aspecten van het geheugen zijn aangedaan. Aangaande de geheugenstoomissen van depressieve patienten wordt over het algemeen aangenomen dat deze met name gelegen zijn in factoren die niet direct te maken hebben met geheugen, zoals veranderingen in de motivatie en een verschil in het gebruik van strategieën. Dergelijke factoren spelen een rol op taken, waarbij zgn. gecontroleerde processen een rol spelen. Gecontroleerde processen zijn processen die onder de bewuste aandacht van de persoon staan, ze verbeteren door oefening, ze worden beïnvloed door het gebrulk van strategieën en factoren als vermoeidheid, motivatie e.d. kunnen van invloed zijn op de prestatie. Een hoger opleidingsniveau correleert over het algemeen positief met gecontroleerde processen, leeftijd is meestal negatief gecorreleerd met dit type processen. Gecontroleerde processen staan in contrast met de zgn. automatische processen. Deze kunnen niet (meer) door oefening verbeterd worden, ze zijn strategie-onafhankelijk, en, eenmaal in gang gezet, zijn ze niet meer te onderbreken. Opleidingsniveau en, per definitie, leeftijd hebben geen invloed op het verloop ervan. Automatische processen spelen vooral een rol bil zogenaamde primingtaken. Voor het onderzoek, beschreven in dit proefschrift, werd gebruik gemaakt van een dergelijke priming-taak, to weten: de perceptuele identificatle taak.

In de perceptuele identificatie taak worden woorden zeer kort (in de orde van enkele tientallen milliseconden) op een scherm gepresenteerd. In een base-line procedure wordt voor leder individu apart bepaald hoe lang de woorden op het scherm moeten blijven om een kans van $40 \%$ correcte identificatie te verkrijgen. Zo is de kans dat een woord bij eerste aanbieding goed geïdentificeerd wordt voor elke proefpersoon min of meer gelijk. In de testfase worden een aantal woorden herhaald. Herhaling van de woorden resulteert in het zgn. facilitatie effect: het verschil in percentage correcte identificatie bil] tweede aanbieding en dat bij eerste aanbieding van de woorden. Dit facilitatie effect zou een weerspiegeling zijn van de automatische retrieval van een episode (een gebeurtenis, die is ingebed in een context van tijd en plaats).

Het patiënt gebonden onderzoek vond plaats in het ziekenhuis van Helmond (gedurende de loop van het onderzoek is de naam van dit zlekenhuis driemaal veranderd: van "het Lambertus ziekenhuis", naar "het Streekziekenhuis Helmond/ 
Deurne" tot "het Elkerliek ziekenhuis"). De meeste controllegroepen werden getest op de vakgroep Neuropsychologie en Psychobiologie van de Rijksuniversiteit Limburg te Maastricht.

\section{Experiment 1}

In het eerste experiment werd de prestatie op een perceptuele identificatie taak van een groep matig ernstige DAT patienten vergeleken met die van een controle groep; op leeftijd en opleiding gematchte, nommale ouderen. Omdat aangenomen werd dat nagenoeg alle aspecten van het geheugen worden aangetast door DAT, werd verondersteld dat de patientgroep minder facilitatie zou vertonen dan de controle groep. (let wel: automatische processen worden, per definitie, niet negatief beinvloed door leeftijd). Daarnaast werd een standaard herkenningstaak afgenomen.

In deze taak werd, indien een woord niet of foutief geildentificeerd was, auditieve feedback gegeven. In tegenstelling tot wat verwacht werd, vertoonden DAT patiënten en normale ouderen nagenoeg evenveel facilitatie. De groepen verschilden wel in de prestatie op de standaard herkenningstaak: DAT patienten herkennen minder woorden dan controles. Echter, het facilitatle effect van beide groepen was kleiner dan verwacht.

\section{Experiment 2}

Ter controle van de vooronderstelling dat het automatische proces, dat ten grondslag ligt aan het facilitatie effect, onafhankelijk van leeftijd is, werd een aanvullend experiment gedaan. Hiervoor werd een groep normale ouderen vergeleken met jonge, normale proefpersonen middels dezelfde perceptuele identificatle procedure. De groep jonge proefpersonen bleek meer facilitatie te vertonen dan de groep oudere proefpersonen Ook was er een verschil in herkenning van de woorden.

\section{Experiment 3}

Parallel aan de hierboven beschreven experimenten liep een experiment waarin licht tot matig ernstige DAT patiënten vergeleken werden met een groep depressieve patiënten en een groep controle patiënten. De controlegroep bestond uit personen, die voor een screening van hun geheugen naar het zliekenhuis kwamen en uit patiënten van de afdeling neurologie, waarbij geen duidelijke geheugenstoornissen geobjectiveerd konden worden.

Overeenkomstig met wat in experiment 1 gevonden werd, kon ook in dit experiment geen verschil in de mate van facilitatie worden vastgesteld tussen de DAT patiënten en de controle groep. Bovendien was er geen verschil in facilitatie tussen de DAT patiënten en de groep depressleve patiënten. Wat betreft de resultaten van de standaard herkenningstaak was er slechts een marginaal verschil te constateren tussen de DAT en depressieve patiënten enerzijds en de controle patiënten anderzljds.

De resultaten van de eerste drie experimenten waren tegengesteld aan wat verwacht werd:

1) de DAT patiënten vertoonden een met de depressleve patiënten en met de normale ouderen vergelijkbaar facilltatie effect op deze priming taak;

2) het facilitatie effect leek mogelijk leeftijdsafhankelijk.

Op basis van de resultaten van experiment 2, werd de vraagstelling van het project uitglebreid naar de effecten van normale veroudering. In de resterende experimenten 
Werden patientgroepen en in leeftijd en opleiding gematchte controle groepen getest. Daarnaast werden ook telkens normale proefpersonen, waarblj de groepen verschillden in leeftijd, met elkaar vergeleken.

\section{Experiment 4}

De mate van facilitatie was, zoals beschreven, minder groot dan verwacht werd. Dat het facilitatie effect gering was kon meerdere oorzaken hebben. Een mogelijke verklaring is wellicht gelegen in de bepalling van de base-line aanbiedingstijd. Wegens de beperkingen van de monitor kon de aanbiedingsduur slechts opgehoogd worden met intervallen van 20 milliseconden. Mogelijk dat hierdoor het facilitatie effect van de diverse groepen op een kwalitatief verschillende wijze beinviloed werd. Daarnaast is bekend dat, indien de eerste aanbieding van een stimulus middels een andere modaliteit wordt waargenomen dan de tweede, het resulterende facilltatie effect kleiner is dan wanneer de beide presentaties binnen dezelfde modaliteit worden aangeboden. Een derde mogelijke verklaring kan mogelijk gezocht worden in de geringe grootte van de aangeboden stimuli, wat de oudere groepen mogelijk nadelig beinvloedde. Mogelijk dat een eventueel verschil tussen de beide patiëntgroepen enerzijds en de normalle ouderen anderzijds verloren gaat in een "floor-effect".

Om bovenstaande bezwaren te ondervangen werd voor de volgende experimenten een scherm met LED-displays ontwikkeld waarmee de stimulus presentatieduur veel nauwkeuriger was aan te sturen. Bijkomend voordeel was dat de lletters duidelijk groter en helderder waren dan van de in de voorgaande experimenten gebruikte monitor. En er werd gebruik gemaakt van een perceptuele identificatie taak, waarbij visuele feedback gegeven werd.

Licht tot matig ernstige DAT patiënten werden vergeleken met een groep depressieve ouderen en een groep gezonde ouderen. Het facilitatie effect dat als gevolg van de veranderde procedure optrad was beduidend groter dan in de voorgaande experimenten. Maar, opnieuw bleek er geen algemeen verschil tussen de patiënt groepen enerzijds en de controle groep anderzijds te bestaan. Enkel wat betreft de facilltatie van laagfrequente woorden bleek een verschil: de groep DAT patiënten vertoonden minder facillitatie dan de groep depressieve patiënten (controles en depressieve patiënten verschilden niet).

\section{Experiment 5}

Om te onderzoeken of er een leeftijsggerelateerde afname in de mate van facilitatie aan te tonen was met de gemodificeerde procedure en apparatuur, werd een experiment uitgevoerd, waarbij zes groepen gezonde proefpersonen, variërend in leeftljd tussen de 20 en de 80 jaar, met elkaar werden vergeleken. Alhoewel er een lichte afname in het facilitatie effect was waar te nemen, verschilden de zes leeftijdsgroepen niet van elkaar.

\section{Experiment 6}

In dit experiment werd bij drie leeftijdsgroepen een perceptuele Identificatio taak afgenomen, waarbij bestaande woorden het stimulusmateriaal vormden en een taak waarbil nonwoorden als stimuli dienden. Drle verschillende leeftijdsgroepen werden getest.

Over het algemeen wordt aangenomen dat de geheugenstoornissen die gepaard gaan met veroudering met name in het episodische domein gelegen zijjn. In tegenstelling tot reguliere woorden bestaat er voor nonwoorden geen semantische representatie. Facilltatie van nonwoorden kan derhalve slechts op basis van episodische processen tot 
stand komen. Indien de afname in facilltatie, die werd waargenomen op de auditieve feedback taak, gelegen zou zijn in een stoomis in episodische processen werd een leettijdsafhankelijke afname in facilitatie verwacht wat betreft de nonwoorden. De drie leefthidsgroepen blijken echter een vergelijkbare prestatie op de taak met nonwoorden te vertonen.

De resultaten tot nu toe samengevat:

- Er is een leeftjdsgerelateerde afname in het facilitatie effect te observeren indien auditieve feedback gegeven wordt.

- Er is geen leeftijdsgerelateerde afname in het facilitatie effect te observeren indien visuele feedback gegeven wordt.

- De leeftijdsgerelateerde afname in het facilitatie effect is niet gelegen in een stoornis van episadische processen.

- Patiëntgroepen verschillen onderling niet in de mate van facilitatie, noch verschillen zij] van normale ouderen.

\section{Experiment 7}

De wijze waarop feedback geboden werd, was echter niet het enige punt waarop de perceptuele identificatie taken gepresenteerd in de voorafgaande hoofdstukken verschilden. Ook de wijze waarop de base-line presentatie duur bepaald werd en de apparatuur verschilde. Daarnaast zouden verschillen in de experimentele groepen verantwoordelijk geweest kunnen zijn voor het all dan niet optreden van een leeftijdsgerelateerde afname in facilitatie. Daarnaast was niet duidelijk hoe feedback het tot stand komen van een facilitatie effect beïnvloedt.

In de experimenten gepresenteerd in hoofdstuk 4, werden drie perceptuele identificatie taken (met auditieve, visuele of zonder feedback) aan alle proefpersonen aangeboden. Op deze wijze varieerde de factor teedback binnen subjecten. Voor alle drie de taken werd het LED-display gebruikt.

In experiment 7 werd de prestatie van een groep DAT patiënten vergeleken met die van een controle groep. Uitgaande van wat tot nu tot gevonden werd, was de verwachting dat beide groepen een vergelijkbare mate van facilitatie zouden vertonen. Deze verwachting werd bevestigd. Een onverwachte bevinding was echter dat, indien er geen feedback gegeven werd, er ook geen facilitatie optrad.

\section{Experiment 8}

Drie leeftijdsgroepen werden op dezelfde wijze getest als hierboven beschreven. Indien de verandering in apparatuur en de procedure van de bepaling van de base-line presentatie duur van invloed zou zijn op het al dan niet optreden van een leeftijdsverschil, werd op geen van de drie taken een leeftijdseffect verwacht.

Normale ouderen verschilden echter van de twee jongere groepen in de mate van facilitatie op de auditieve feedback taak. Op de visuele feedback taak werd ook nu geen leeftijdsgerelateerde afname in facilitatie waargenomen. De grootte van de facilitatie effecten kwam in hoge mate overeen met die van de eerdere experimenten. Opnieuw opvallende was het ontbreken van een facilitatie effect op de taak waarbij geen feedback gegeven werd. Blijkbaar was een enkele, zeer korte aanbieding van de woorden niet voldoende om een facilitatie effect te doen optreden.

De resultaten van de experimenten 7 en 8 samengevat:

- Er is een leeftijdsgerelateerd verschil in facilitatie op de auditieve feedback taak, dat niet als gevolg van artefacten met betrekking tot procedure of apparatuur 
verklaard kam worden.

- Een dergelijk leeftijdsgerelateerd verschil treedt niet op indien visuele feedback gegeven wordt.

- Er treedt geen facilitatie effect op, indien er geen feedback gegeven wordt na incorrecte identificatie van een woord.

- Op geen van deze taken is er een verschil in prestatie tussen DAT patiënten en normale ouderen.

\section{Experiment 9}

De resultaten van de hierboven beschreven experimenten in aanmerking genomen, lijkt de conclusie gerechtvaardigd, dat facillitatie enkell optreedt indien or feedback wordt gegeven. Aldus, kan men de presentatie van feedback eigenlijk beschouwen als de eerste relevante presentatie van een stimulus. De uiteenlopende resultaten van de auditieve en de visuele perceptuele identificatie taken moeten worden toegeschreven aan de verschillen in procedure. Het eerste verschil is het al of niet veranderen van modaliteit, waarbinnen de stimuli aangeboden en verwerkt worden. Een tweede verschil is de duur van de eerste (liet wel: de feedback) presentatie. In de auditieve taak wordt de feedback aangeboden gedurende de tijd die nodig is om het woord uit te spreken. In de visuele feedback taak wordt het woord zolang aangeboden als nodig is om het woord te analyseren en uit te spreken. Mogelijk dat ouderen sensitiever zijn voor verandering van modaliteit tussen eerste en tweede aanbleding, of gevoeliger voor verschillen in presentatie duur van de stimulus. Om deze mogelijkheden te onderzoeken werd een nileuwe perceptuele identificatie taak (de interval taak) ontwikkeld.

In deze taak was de wijze waarop de eerste presentatie van stimull plaats vond vergelijkbaar met die van de visuele feedback taken. Er werd ruimschoots de gelegenheid gegeven de woorden te analyseren en uit te spreken, onder het mom van een reactietijd meting. Het percentage correcte identificatie bij herhaalde presentatie werd na drie verschillende intervalien $(0,15$ en 45 minuten) gemeten. Op deze wijze kon ook het verloop van het facilitatie effect over tijd geëvalueerd worden.

De prestatie van een groep DAT patiënten werd vergeleken met die van een groep normale ouderen. Omdat in de voorgaande experimenten geen verschil tussen deze groepen aangetoond konden worden, werd ook nu geen verschil verwacht. deze hypothese werd bevestigd door de resultaten.

\section{Experiment 10}

Vervolgens werd de interval taak afgenomen bij drie groepen, in leeftijd variarende;

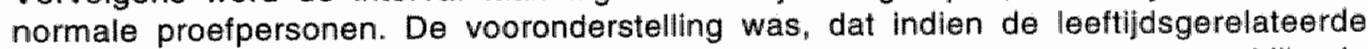
verschillen die tot nu toe geobserveerd werden het gevolg waren van een verschillende gevoeligheid van de ouderen voor veranderingen in modaliteit of presentatleduur, er geen verschil in de mate van facilltatie zou optreden op deze taak.

Echter, in tegenstelling tot wat verwacht werd, verschilden de groepen wel degelljk van elkaar: de oudere groep vertoonde minder facilitatie dan de twee jongere groepen, zowel na een interval van 0 minuten, als na een interval van 15 minuten. Op het laatste interval (45 minuten) was het onderscheid verdwenen. Het geobserveerde facllitatle effect nam voor alle groepen van het eerste naar het tweede interval nogall sterk af. De resultaten worden besproken in relatie tot het onderscheid tussen episodisch en semantisch geheugen, alsmede in relatle tot de algehele vertraging van cognitieve processen bij veroudering. 


\section{Hoofdstuk 6}

In dit hoofdstuk worden de diverse bevindingen van deze studie besproken in het kader van de in de inleiding beschreven theoretische kaders. In relatie tot de discussie of de oorsprong van het facilitatie effect gelegen is in episodische dan wel in semantische informatieverwerking, wordt geconcludeerd dat:

de relatieve bijdrage van elk type verwerking, of processen afhankelijk is van de speciffeke taakvariabelen.

Vervolgens wordt de discussle binnen dit kader nader toeglespitst op een mogelijk mechanisme, dat verantwoordelijk zou kunnen zijn voor de in deze studie geobserveerde leeftijdsafhankelijke verschillen. De bevindingen worden besproken aan de hand van het model van Salasoo et al. (1985) en een meer recent model van Masson en MacLeod (1992). In dit model wordt een mogellik interactioneel proces voorgesteld tussen aspecten met betrekking tot de verwerking context en aspecten met betrekking tot de verwerking van de betekenis van woorden. Een mogelijke verklaring van de in deze studie gevonden resultaten zou kunnen zijn dat oudere subjecten minder geneigd zijn tot zelf geïnitieerde operaties ten bate van het onderscheiden van de verschillende betekenissen van woorden.

Vervolgens worden de resultaten belicht in het kader van automatische en gecontroleerde processen. Dit leldt tot de conclusie dat:

het criterium van de leeftijdsonathankelijkheid van automatische processen dient nader beschouwd te worden. De resultaten van de onderhavige studie, alsmede die van andere recente studies tonen ofwel aan dat automatische processen niet altijd onaangetast zijn door leeftijd, ofwel dat processen die tot op heden als automatisch gelabeld werden dit etiket niet verdienen.

De discussie met betrekking tot het onderscheid tussen impliciet en expliciet geheugen, leidt tot de aanbeveling specifleke taakvariabelen aan een nadere inspectie te onderwerpen in plaats van steeds nieuwe concepten toe te voegen aan de toch al zo ingewikkelde discussie met betrekking tot de oorsprong van het geheugen. De conclusie in relatie tot het onderscheld impliclet/expliciet luidt:

de term impliciet geheugen, op welke wijze ook geinterpreteerd, kan niet beschouwd worden als een vastomschreven concept.

De algehele conclusies van de onderhavige studie zijn als volgt samen te vatten:

- DAT patiënten verschillen niet in de mate van facilitatie in de perceptuele identificatie taken gebruikt in deze studie. Dit geldt zowel in vergelijking tot normale ouderen, als in vargelljking met depressieve ouderen. Als zodanig kunnen de perceptuele identificatie taken zoals ze hier worden gepresenteerd niet bijdragen in de ontwikkeling van een nuttig instrument in de praktijk van de differentiaal diagnostiek.

- Het criterium van leeftijdsonafhankelijkheid van automatische processen, voorgesteld door Hasher en Zacks (1979) is onhoudbaar en dient opnieuw te worden bezien.

- Een systematische variatie van diverse taakvariabelen plaatst het nut van het onderscheid tussen impliciet ene expliciet geheugen in een nieuw licht. 
- Het paradigma van perceptuele identificatie vormt een veelbelovend en interessant kader voor het onderzoek naar de fundamentele mechanismen die ten grondslag kunnen liggen aan de verslechterde geheugenprestatie van ouderen. 


\section{REFERENCES}

Abbenhuis, M.A., Raaijmakers, W.G.M., Raaijmakers, J.G.W. \& van Woerden, G.J.M. (1990). Episodic memory in Dementia of the Alzheimer Type and in normal ageing: Similar impairment in automatic processing. The Quarterly Journal of Experimental Psychology: 42A(3), 569-583.

Anders, T.R. , Fozard, J.L. \& Lillyquist, T.D. (1972). Effects of age upon retrieval from shortterm memory. Developmental Psychology, 6(2), 214-217.

Anderson, J.R. (1983). A spreading activation theory of memory. Journal of Verbal Learning and Verbal Behavior, 22, 261-295.

Arbuckle, T.Y."Vanderleck, V.F., Harsany, M. \& Lapidus, L. (1990). Adult age differences in memory in relation to avallability and accessibility of knowledge-based schemas. Journar of Experimental Psychology: Learning, Memory, and Cognition, 16(2), 305-315.

Balota, D.A. (1983). Automatic semantic activation and episodic memory encoding. Journal of Verbal Learning and Verbal Behavior, 22, 88-104.

Balota, D.A. \& Duchek, J.M. (1988). Age-related differences in lexical access, spreading activation, and simple pronunciation. Psychology and Aging, 3(1), 84-93.

Bassili, J.N., Smithe, M.C. \& MacLeod, C.M. (1989). Auditory and visual word-stem completion: Separating dat-driven and conceptually driven processes. The Quarterly Journal of Experimental Psychology, 41.A, 439-453.

Begg, I. \& Snider, A. (1987). The generation effect: Evidence for generalized inhibition. Journal of Experimental Psychology: Learning, Memory, and Cognition, 13, 553-563.

Bird, M. \& Luszoz, M. (1991). Encoding specificity, depth of processing and cued recall in Alzheimer's disease. Journal of Clinical and Experimental Psychology, 13(4), 508-520.

Bondi, M.W. \& Kaszniak, A.W. (1991). Implicit and explicit memory in Alzheimer's disease and Parkinson's disease. Journal of Clinical and Experimental Neuropsychology, 13(2), 339358.

Butters, N. (1984). The clinical aspects of memory disorders: Contributions from experimental studies of amnesia and dementila. Journal of Clinical Neuropsychology, $6(1), 17-36$.

Burke, D.M., White, H. \& Diaz, D.L. (1987). Semantic priming in young and older adults: Evidence for age constancy in automatic and attentional processes. Journal of Experimental Psychology: Human Perception and Performance, 13, 79.88.

Cerella, J. (1985). Age-related decline in extrafoveal letter perception. Journal of Gerontology, $40,727-736$.

Cerella, J, Poon, L.W. \& Fozard, J.L. (1982). Age and iconic read-out. Journal of Gerontolagy, $37(2), \quad 197-202$.

Cermak, L.S., Talbot, N. Chandler, K. \& Wolbarst, L.R. (1985). The perceptual priming phenomenon in amnesia. Neuropsychologia, 23, 615-622. 
Chertkow, H. \& Bub, D. (1990). Semantic memory loss in Dementia of the Alzheimer's Type: What do various measures measure?. Brain, 113, 397-417.

Chiarello \& Hoyer, (1988). Adult age differences in implicit and explicit memory: Time course and encoding effects. Psychology of aging, 3, 358-366.

Cohen, N.J. \& Squire, L.R. ( 1980$)$. Preserved learning and retention of pattern analyzing skill in amnesia: Dissociation of knowing how and knowing that. Science, 210, 207-210.

Cohen, R.M. Weingartner, H., Smallberg, S.A Pickar, D. \& Murphy, D.L. (1982). Effort and cognition in depression. Archives of General Psychiatry, 39, 593-597.

Corkin, S. (1982). Some relationships between the global amnesias and the memory impairments in Alzheimer's disease. In: S.Corkin, K.L. Davis, J.H. Growdon, E. Usdin \& R.J. Wurtman (Eds.), Alzheimer's disease: A report of progress in research. (pp. 149-164). New York: Aaven Press.

Craik, F.I.M. (1977). Age differences in human memory. In: J.E. Birren \& K.W. Schaie (Eds.). Harrabook of the Psychology of Aging (pp. 384-420). New York: Van Rostrand Reinhold Company.

Craik, F.I.M. (1983). On the transfer of information from temporary to permanent memory. In: D.E. Broadbent (Ed.), Functional Aspects of Memory. London: Royal Society.

Craik, F.I.M. (1986). A functional account of age differences in memory. In: F. Klix \& $H$. Hagendorf (Eds.). Human Memary and Cognitive Capabilities: Mechanisms and Performances (pp. 409-422). Amsterdam: Elsevier Science Publishers B.V.

Craik, F.I.M. \& Byrd, M. (1982). Aging and cognitive deficits: The role of attentional resources. In: F.I.M. Craik \& S. Trehub (Eds.), Aging and cognitive processes (pp. 191-211). New York: Plenum Press.

Craik, F.l.M. \& Rabinowitz, J.C. (1984). Age differences in the acquisition and use of verbal information: A tutorial review. In: H. Bouma and D.G. Bouwhuis (Eds.), Attention and Performance. $X$. Control of Language Processes (pp. 471-499). Hillsdalle, Nul: Erlbaum.

Craik, F.I.M. \& Simon, E. (1980). Age differences in memory: The roles of attention and depth of processing. In: L.W. Poon, J.L. Fozard, L.S. Cermak, D. Arenberg \& L.W. Thompson (Eds.), New Directions in Memory and Aging (pp. 95-112). Hillsdale, NJ: Lawrence Erlbaum Assac.

Cummings, J.L.. Houlihan, J.P. \& Hill, M.A. (1986). The pattern of reading deterioration in dementia of the Alzheimer type: Observations and implications. Brain and Language, 29, 315* 323.

Davis "H.P., Cohen, A., Gandy, M., Colombo, P., vanDusseldorp, G., Simolke, N. \& Romano, J. (1990). Lexical priming deficits as a function of age. Behavioral Neuroscience, 104(2), $288-297$.

Denney, N.W., Miller, B.V., Dew, J.R. \& Levav, A.L. (1991). An adult developmental studly of contextual memory. Journal of Gerontology: Psychological Sciences, 46(2), 44-50.

Diagnostic and Statistical Manual of Mental Disorders (1980). Washington, DC: American Psychiatric Association, 3rd ed. 
Diagnostic and Statistical Manual of Mental Disorders-Revised (1987). Washington, DC: American Psychiatric Association, .

Diamond, R. \& Rozin, P. (1984). Activation of existing memories in anterograde amnesia. Journal of Abnormal Psychology, 93, 98-105.

Ellis, H.C., Thomas, R.L. \& Rodriguez, U.A. (1984). Emotional mood states and memory: Elaborative encoding, semantic processing, and cognitive effort. Journal of Experimental Psychology: Learning, Memory, and Cognition, 10, 470-482.

Feinberg, T. \& Goodman, B. (1984). Affective illness, dementia and pseudodementia. Journal of Clinical Psychiatry, 45, 100-103.

Feustel, T.C., Shiffrin, R.M. \& Salasoo, A. (1983). Episodic and lexical contributions to the repetition effect in word identification. Journal of Experimental Psychology: General, 112 . $309-346$.

Forster, K.I. \& Dawis, C. (1984). Repetition priming and frequency attenuation in lexical access. Journal of Experimental Psychology: Learning, Memory, and Cognition, 10, 680-698.

Gabrielii, لl.D.E. (1989). Dissociation of memory capacities in neurodegenerative disease. In: Proceedings of the Fifth Meeting of the International Study Group on the Pharmacology of Memory Disorders Associated with Aging. Zurich, Switzerland, January 20-22.

Graf, P. \& Mandler, G. (1984). Activation makes words more accessible, but not necessarily more retrievable. Journal of Verbal Learning and Verbal Behavior, 23, 553-568.

Graf, P. \& Schacter, D.L. (1985). Implicit and explicit memory for new associations in normal and amnesic subjects. Journal of Experimental Psychology: Learning, Memory, and Cognition, 13, 45-53.

Graf, P., Shimamura, A.P. \& Squire, L.R. (1985). Priming across modalities and priming across category levels: Extending the domain of preserved function in amnesia. Journal of Experimental Psychology: Leaming, Memory, and Cognition, 11, 385-395.

Graf, $P_{. y}$ Squire, L.R. \& Mandler, G. (1984). The information that amnesic patients do not forget. Joumal of Experimental Psychology: Leaming, Memory, and Cognition, 10, 164-178.

Grafman, J., Weingartner, H., Newhouse, P.A., Thompson, K., Lalonde, F., Litvan, I., Molchan, $S$. \& Sunderland, T. (1990). Implicit learning in patients with Alzhelmer"s disease. Pharmacopsychiatry, 23, 94-101.

Grober, E., Buschke, H., Chrystal ${ }_{1}$., Bang, S. \& Dresmer, R. (1988). Screening for dementia by memory testing. Neurology, 38, 900-903.

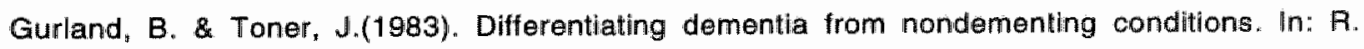
Mayeux and W.G. Rosen, The Dementias. New York: Raven Press.

Hart, R.P., Kwentus, J.A., Hamer, R.M. \& Taylor, J.R. (1987a). Selective reminding procedure in depression and dementia. Psychology and Aging, 2, 111-115.

Hart, R.P., Kwentus, J.A., Taylor, J.R. \& Harkins, S.W. (1987b). Rate of forgetting in dementia and depression. Joumal of Consulting and Clinical Psychology, 55, 101-105.

Hart, R.P., Kwentus, J.A., Wade, J.B. \& Hamer, R.M. (1987c). Digit symboll performance in mild 
dementia and depression. Journal of Consulting and Clinical Psychology, 55, 236-238.

Hart, R.P. Kwentus, J.A., Wade, J.B. \& Taylor, J.R. (1988). Modified Wisconsin Card Sorting Test in elderly normal, depressed and demented patients. The Clinical Neuropsychologist, 2(1), 49-56.

Hart; S. (1985). Explorations of Cognitive Dysfunction in Dementia of Alzheimer Type.

Unpublished Ph. D. thesis, University of Lonidon.

Hart, S. (1988). Language and dementia: A review. Psychological Medicine, 18, 99-112.

Hartley, A.A. MoKenzie, C.R.M. (1991). Attentional and perceptual contributions to the identification of extrafoveal stimuli: Adult age comparisons. Journal of gerontology: Psychological Sclences, 46(5), 202-206.

Hasher, L. \& Zacks; R.T. (1979). Automatic and effortful processes in memory. Journal of Experimental Psychology: General, 108, 356-388.

Heindel, W.C. Salmon, D.P., Shults, C.W., Walicke, P.A. \& Butters, N. (1989). Neuropsychological evidence for multiple implicit memory systems: A comparison of Alzheimer's, Huntington's, and Parkinson's patients. Journal of Neuroscience, 9(2), 582587.

Herlitz, A. \& Vitianen, M. (1991). Semantic organization and verbal episodic memory in patients with mild and moderate Alzheimer's Disease. Journal of Clinical and Experimental Neuropsychology, 13(4), 559-574.

Hertel, P.T. \& Hardin, T.S. (1990). Remembering with and without awareness in a depressed mood: Evidence of deficits in initiative. Journal of Experimental Psychology: General, $119(1), 45-59$.

Hess, T.M. \& Arnould, D. (1986). Adult age differences in memory for explicit and implicit sentence information. Journal of Gerontology, 41(2), 191-194.

Hinton, D.F., Sadun, A.A., Blanks, J.C. \& Miller, C.A. (1986). Optic-nerve degeneration in Alzheimer's disease. New England Journal of Medicine, 315, 485-487.

Howard, D.V. MoAndrews, M.P. \& Lasaga, M.I. (1981). Semantic priming of lexical decisions in young and old adults. Joumal of Gerontology, 36(6), 707-714.

Howard, D.V., Shaw, R.J. \& Helsey, J.G. (1986). Aging and the time course of semantic activation. Journal of Gerontalogy, 41, 195-203.

Hultsch, Masson \& Small, (1991). Adult age differences in direct and indirect tests of memory. Jounal of Gerontology: Psychological Sciences, 46(1), 22-30.

Hunt, R.R. \& Toth, J.P. (1990). Perceptual identification, fragment completion, and free recall: Concepts and data. Journal of Experimental psychology: Learning, Memory, and Cognition, $16(2), \quad 282-290$.

Jacoby, L.L. (1983a). Perceptual enhancement: Persistent effects of an experience. Journal af Experimental Psychology: Learning, Memory, and Cognition, 9, 21-38.

Jacoby, L.L. (1983b), Remembering the data: Analyzing interactive processes in reading. Journal of verbal Learning and Verbal Behavior, 22, 485-508. 
Jacoby, L.L. (1984). Incidental versus intentional retrieval: Remembering and awareness as separate issues. In: L.R. Squire and N. Butters (Eds.) Neuropsychology of Memory (pp. 145156). New York: Guilford Press.

Jacoby, L.L. \& Dallas, M. (1981). On the relationship between autobiographical memory and perceptual learning. Journal of Experimental Psychology: General, 110, 3106-340.

Jacoby, L.L. \& Hayman, C.A.G. (1987). Specific visual transfer in word identification. Joumal of Experimental Psychology: Learning, Memory, and Cognition, 13(3), 456-463.

Jacoby, L.L. \& Witherspoon, D. (1982). Remembering without awareness. Canadian Journal of Psychology, 36, 300-324.

Johnson, M.H. \& Magaro, P.A. (1987). Effects of mood and severity on memory processes in depression and mania. Psychological Bulletin; 101, 28-40.

Johnston, W.A., Dark, V.J. \& Jacoby, L.L. (1985). Perceptual fluency and recognition memory. Journal of Experimental Psychology: Learning, Memory, and Cognition, 11, 3-11.

Kahneman, D. (1973). Attention and Effort. Englewood Cliffs, NJ: Prentice Hall.

Kausler,D.H. \& Hakami, M.K. (1983). Memory for activities: Adult age differences and intentionality. Developmental Psychology, 19(6), 889-894.

Kausler,D.H., Hakami, M.K. \& Wright, R.E. (1982). Adult age differences in frequency judgments of categorical representations. Journal of Gerontology, 37(3), 365-371.

Kausler, D.H., Lichty, W. \& Davis, R.T. (1985). Temporal memory for performed activities: Intentionality and adult age differences. Developmental Psychology, 21(6), 1132-1138.

Kausier, D.H. \& Wiley, J.G. (1990). Temporal memory and content memory for actions: Adult age differences in acquisition and retrieval. Experimental Aging Pesearch, 16(3), 147-150.

Keane, M.M., Gabrieli, J.D.E., Fennema, A.C., Growdon, J.H. \& Corkin, S. (1991). Evidence for a dissociation between perceptual and conceptual priming in Alzheilmer's disease. Behavioral Neuroscience, 105(2), 326-342.

Kausler,D.H., Wright, R.E. \& Hakami, M.K. (1981). Variation in task complexity and adult age differences in frequency-of-occurrence judgments. Bulletin of the Psychomomic Society, $18(4), \quad 195-197$.

Kinsbourne, M. \& Wood, F. (1982). Theoretical considerations regarding the episodic semantic distinction. In: L.S. Cermak (Ed.), Human Memory and Amnesia. Hillsdale, NJ: Lawrenco Erlbaum Associales.

Kolers, P.A. (1976). Reading a year later. Joumal of Experimental Psychology: Human Loaming and Memory, 2, 554-565.

Kopeiman, (1985). Rates of forgetting in Alzheimer-type dementla and korsakoff's syndrome. Neuropsychologia, 23(5), 623-638.

Kopelman, M.D. (1986a). Clinical tests of memory. British Journal of Psychiatry, 148, 517 525.

Kopelman, M.D. (1986b). Recall of anomalous sentences in dementia and amnesia. Brain and Language, 29, 154-179. 
La Rue, A, D'Elia, L.F. Clark, E.O, Spar, J.E. \& Jarvik, L.F. (1986). Clinical tests of memory in dementia, depression and healthy aging: Journal of Psychology and Aging, 1(1), 69-77.

Lazarus, L.W., Newton, N., Cohler, B., Lesser, J. \& Schweon, C. (1987). Frequency and presentation of depressive symptoms in patients with primary degenerative dementia. American Journal of Psychiatry, 144(1), 41-45.

Lezak, M.D. (1983). Neuropsychological Assessment. New York: Oxford, University Press.

Light, L.L. (1991). Memory and aging: Four hypotheses in search of data. Annual Review of Psychology; 42, 333-376.

Light L.L. \& Singh, A. (1987). Implicit and explicit memory in young and older adults. Journal of Experimental Psychology: Learning, Memory, and Cognition, 13(4), 531-541.

Light, L.L., Singh, A. \& Capps, J.L. (1986). Dissociation of memory and awareness in young and older adults. Journal of Clinical and Experimental Neuropsychology, 8(1), 62-74.

Light, L.L., Zelinsky, E.M. \& Moore, M. (1982). Adult age differences in reasoning from new information. Journal of Experimental Psychology: Learning, Memory, and Cognition, $8,435-$ 447.

Madclen, D.J. \& Allen, P.A. (1991). Adult age differences in the rate of information extraction during visual search. Journal of Gerontology: Psychological Sciences, 46(3), 124-126.

Mahendra, B. (1985). Depression and dementia: The multi-faceted relationship. Psychological Medicine, 15, 227-236.

Mandler, G. (1980). Recognizing: The judgment of previous occurrence. Psychological Review, $87(3), \quad 252-271$.

Martin, A. \& Fedio, P. (1983). Word production and comprehension in Alzheimer's disease: The breakdown of semantic knowledge. Brain and Language, 19, 124-141.

Martone, M., Butters, N., Payne, M., Becker, J.T. \& Sax, D.S. (1984). Dissociations between skill learning and verbal recognition in amnesia and dementia. Archives of Neurology, 41 , 965.970 .

Masson, M.E.J. \& MacLeod, C.M. (1992). Reenacting the route to interpretation: Enhanced perceptual identification without prior perception. Journal of Experimental Psychology: General, $121(2), 145-176$.

Moclelland, J.L. \& Rumelhart, D.E. (1985). Distributed memory and the specific representation of general and specific information. Journal of Experimental Psychology: General, 114, 159188.

MeDowd, J.M. \& Oseas-Kreger, D.M. (1991). Aging, inhibitory processes, and negative priming. Journal of Gerontology: Psychological Sciences, 46(6), 340-345.

McKhan, G., Drachman, D., Folstein, M., Katzman, R., Price, D. \& Stadlan, E.M. (1984). Clinical diagnosis of Alzheimer's disease: Report of the NINCDS-ADRDA work group under the auspices of the Department of Health and Human Service task force on Alzheimer's disease. Neurology, 34, $939-944$.

McKoon, G. \& Ratcliff, R. (1986). Automatic activation of episodic information in a semantic 
memory task. Joumal of Experimental Psychology: Learning, Memory, and Cognition, $12(1)$, $108-115$.

McKoon, G. \& Ratcliff, R \& Dell, G. (1986). A critical evaluation of the episodic-semantic distinction. Journal of Experimental Psychology: Leaming, Memory, and Cognilion, 12, 295. 306 .

Miller, E. (1975). Impaired recall and the memory disturbance in presenile dementia. British Journal of Social and Clinical Psychology, 14, 73-79.

Mitchell, D.B. (1989). How many memory systems? Evidence from aging. Journal of Experimental Psychology: Learning, Memory, and Cognition, 15(1), 31-49.

Mitchell, D.B. \& Brown, A.S. (1988). Persistent repetition priming in picture naming and its dissociation from recognition memory. Journal of Experimental Psychology: Learning. Memory, and Cognition, 14(2), 213-222.

Mitchell, D.B., Brown, A.S. \& Murphy, D.R. (1990). Dissociations between procedural and episodic memory: effects of time and aging. Psychology of Aging, 5, 264-276.

Mitchell, D.B., Hunt, R.R. \& Schmitt, F.A. (1986). The generation effect and reality monitoring: Evidence from dementia and normal aging. The Journal of Gerontology, 41(1),79-84.

Morris, R.G. \& Kopelman, M.D. (1986). The memory deficits in Alzheimer-type dementia: A review. The Quarterly Journal of Experimental Psychology, 38A, 575-602.

Morris, R.G., Wheatly, J. \& Britton, P.G. (1983). Retrleval from long-term memory in senile dementia: Cued recall revisited. British Journal of Clinical Psychology, 22, 141-142.

Morton, J. (1969). Interaction of information in word recognition. Psychological Feview, $76(2), \quad 165-178$.

Moscovitch, M. (1982). A neuropsychological approach to perception and memory deficit in normat and pathological aging. In: F.I.M. Craik and S. Trehub (Eds.), Aging and cognitive processes. (pp. 55-79). New York: Plenum Press.

Moscovitch, M., Winocour, G \& McLachlan, D. (1986). Memory as assessed by recognition and reading time in normal and memoryimpaired people with Alzheimer's disease and other neurological disorders. Journal of Experimental Psychology: General, 115, 331.347.

Moss, M.B., Albert, M.S., Butters, N. \& Payne, M. (1986). Differential patterns of memory loss among patients with Alzheimer's disease, Huntington's disease, and alcoholic Korsakofl's syndrome. Archives of neurology, 43, 239-246.

Musen, G. \& Treisman, A. (1990). Implicit and explicit memory for visual patterns, Journal of Experimental Psychology: Learning . Memory, and Cognition, 16(1), 127-137.

Nebes, R.D., Boller, F. \& Holland, A. (1986). Use of semantic context by patients with Alzheimer's disease. Psychology and Aging, $1,261-269$.

Nebes, R.D., Brady, C.B. \& Huff, F.J. (1989). Automatic and attentional mechanisms of semantic priming in Alzheimer's disease. Journal of Clinical and Experimental Neuropsychology. $11(2), \quad 219-230$.

Nebes, R.D., Martin, D.C. \& Horn, L.C. (1984). Sparing of semantic memory in Alzheimer's 
disease. Journal of Abnomal Psychology, 93, 321-330.

Neely. J.H. (1977). Semantic priming and retrieval from lexical memory: Roles of inhibitionless spreading activation and limited-capacity attention. Journal of Experimental Psychology: General, 106(3), 226-254.

Neils, $J_{n}$ Newman, G.W. Hill, M. Weiler, E. (1991). The effects of rate, sequencing, and memory on auditory processing in the elderly: Journal of Gerontology: Psychological Sciences, 46 (2), $71-75$.

Newman, R.P., Weingartner, H., Smallberg, S.A. \& Calne, D.B. (1984). Effortful and automatic memory: Effects of dopamine. Neurology, 34, 805-807.

Ober, B.A., Drankers, N.F., Koss, E., Dellis, D.C. \& Friedland, R.P. (1986). Retrieval from semantic memory in Alzheimer-type dementia. Journal of Clinical and Experimental Neuropsychology, 8(1), 75-92.

O'Hara, M.W., Hinrichs, J.V., Kohout, F.J., Wallace, R.B. \& Lemke, J.H. (1986). Memory complaints and memory performance in the depressed ellderly. Psychology and Aging, 1, 208214 .

Oliphant, G.W. (1983). Repetition and recency effects in word recognition. Australian dournal of Psychology, 35(3), 393-403.

Park, D.C., Smith, A.D., Dudley, W.N., \& La Fronza, V.N. (1989). Effects of age and a divided attention task presented during encading and retrieval on memory. Journal of Experimental Psychology: Learning, Memory, and Cognition, , 15, 1185-1191.

Parkin. A.J., Reid, T.K. \& Russo (1990). On the differential nature of implicit and explicit memory. Memory \& Cognition, 18(5), 507-514

Partridge, F.M., Knight, R.G. \& Feehan, M. (1990). Direct and indirect memory performance in patients with senile dementia. Psychological Medicine, 20, 111-118.

Perruchet, P. \& Baveux, P. (1989). Correlational analysis of explicit and implicit memory performance. Memory \& Cognition, 17(1), 77-86.

Posner, M.I. \& Snyder, C.R.R. (1975). Attention and cognitive control. In: R.L. Solso (Ed.), Information Processing and Cognition (pp. 55-85). Hillsdale, NJ: Erlbaum.

Puglisi, J.T., Park, D.C., Smith, A.D. \& Dudley, W.N. (1988). Age differences in encoding specificity. The Journals of Gerontology: Psychological Sciences, 43, 145-150.

Rabbit P.M.A. (1982). How do old people know what to do next? In: F.I.M. Craik \& S. Trehulb (Eds.), Aging and cognitive processes. New York: Plenum Press.

Rabbit. P.M.A. (1984). How old people prepare themselves for events which they expect. In: $H$. Bouma \& D.G. Bouwhuis (Eds.), Attention and performance $X$ (pp. 515-527). Hillsdalle "Nu: Lawrence Ertbaum Associates Inc.

Rabinowitz, J.C. \& Ackerman, B.P. (1982). In: F.I.M. Cralk and S. Trehub (Eds.), Aging and Cognitive Processes. (pp. 145-154). New York: Plenum Press.

Randolph, C. (1991). Implicit, explicit, and semantic memory functions in Alzheimer's disease and Huntington's disease. Journal of Clinical and Experimental Neuropsychology, 13(4), 
$479-494$

Rankin, J.L. \& Hinrichs, J.V. (1983). Age, presentation rate, and the effectiveness of structural and semantic cues. The Journal of Gerontology, 38, 593-596.

Reder, L.M., Wible, C. \& Martin, J. (1986). Differential memory changes with age: Exact retrieval versus plausible inference. Joumal of Experimental Psychology: Learning, Momory, and Cognition, 12, 72-81.

Reisberg, B., Ferris, S.H., de Lean, M.J. \& Crook, T. (1982). The Global Deterioration Scale for assessment of primary degenerative dementia. American Joumal of Psychiatry, 139, 1136. 1139.

Richardson-Klavehn, A. \& Bjork "R.A. (1988). Measures of memory. Annual Review of Psychology, 39, 475-543.

Roediger, H.L. III \& Blaxton, T.A. (1987). Retrieval modes produce dissociations in memory for surface information. In: D.S. Gorfein \& R.R. Hoffman (Eds.), Memory and Cognitive Processes: The Ebbinghaus Centennial Conference (pp. 349-379). Hillsdale, NJ: Erlbaum.

Rose, Yesavage, Hill \& Bower, (1986). Priming effects and recognition memory in young and elderly adults. Experimental Aging Research, 12, 31-37

Rosenfield, I. (1988). The Invention of Memory: A New View of the Brain. New York: Basic Books.

Roy-Byrne, P.P., Weingartner, H., Bierer, L.M., Thompson, K. \& Post, R.M. (1986). Effortful and automatic processes in depression. Archives of General Psychiatry, 43, 265-267.

Salasoo, A., Shiffrin, R.M. \& Feustel, T.C. (1985). Building permanent memory codes: Codification, and repetition effects in word identification. Journal of Experimental Psychology: General, 114, 50-77.

Salmon, D.P., Shimamura, A.P., Butters, N. \& Smith, S. (1988). Lexical and semantic priming deficits in patients with Alzheimer's disease. Journal of Clinical and Experimental Neuropsychology, 10, 477-494.

Salthouse, T.A. (1988). Resource-reduction interpretations of cognitive aging. Developmental Review, 8(3), 238-272.

Salthouse, T.A., Mitchell, D.R.D., Skovronek, E. \& Babcock, R.L. (1989). Effects of adult age and working memory on reasoning and spatial abilities. Journal of Experimental Psychology: Learning, Memory, and Cognition, (15), 507-516.

Schacter, D.L. (1985). Priming of old and new knowledge in amnesic patients and normal subjects. Annals of the New Yark Academy of Sciences, 444, 41-53.

Schacter, D.L. (1987). Implicit memory: History and current status. Journal of Exporimental Psychology: Learning, Memory, and Cognition, 13(3), 501 518.

Schacter, D.L. \& Graf, P. (1986a). Effects of elaborative processing on implicit and explicit memory for new associations. Journal of Experimental Psychology: Learning. Memory, and Cognition, 12, 432-444.

Schacter, D.L. \& Graf, P. (1986b). Preserved learning in amnesic patients: Perspectives from research on direct priming. Journal of Cinical and Experimental Neuropsychalogy, 8, 727- 
743.

Schacter, D.L. \& Tulving, E: (1982), Memory, amnesia, and the episodic/semantic distinction. In: R.L. Isaacson \& N.E. Spear (Eds), The Expression of Knowledge (pp. 33-65). New York: Plenum Press.

Schläpfer, T.E., Groner, M., Lavoyer, E. \& Fisch, HI-U. (1991). Visual masking: A reliable measure for the assessment of cognitive dysfunction in the elderly? Journal of gerontology: Psychological Sciences, 46(4), 157-161.

Schneider, W. \& Shiffrin, R.M. (1977). Controlled and automatic human information processing: I Detection, search, and attention. Psychological Review, 84, 1-66.

Shiffrin, F.M. \& Schneider, W. (1977). Controlled and automatic human information processing: II Perceptual learning, automatic attending, and a general theory. Psychological Review, 84, $127-190$.

Shimamura, A.P. (1986), Priming effects in amnesia: Evidence for a dissociable memory function. The Quarterly Journal of Experimental Psychology, 38A, 619-644.

Shimamura, A.P. \& Squire, L.P. (1984). Paired associate learning and priming effects in amnesia: A neuropsychological study. Journal of Experimental Psychology: General, 11, 556570.

Shimamura, A.P., Salmon, D.P., Squire, L.R. \& Butters, N. (1987). Memory dysfunction and word priming in dementia and amnesia. Behavioral Neuroscience, 101(3), 347-351.

Silberman, E.K., Weingartner, H., Laraia, M., Byrnes, S. \& Post, M. (1983). Processing of emotional properties of stimuli by depressed and normal subjects. The Journal of Nervaus and Mental Disease, 171, 10-14.

Squire, L.R. (1982). The neuropsychology of human memory. American Review of Neuroscience, $5,241-273$.

Squire, L.R. \& Cohen, N.J. (1984). Human memory and amnesia. In: J. McCaugh, G., G. Lynch \& N. Weinberger (Eds.). Proceedings of the Conference on the Neurobiology of Learning and Memory. New York: Gullford Press.

Squire, L.R., Shimamura, A.P. \& Graf, P. (1985). Independence of recognition memory and priming effects: A neuropsychological analysis. Journal of Experimental Psychology: Learning, Memory " and Cognition, 11, 37-44.

Stanowitch, K.E. \& West, R.F. (1983). On priming by a sentence context. Journal of Experimental Psychology: General, 112(1), 1-36.

Strauss, M.E., Weingartner, H. \& Thompson, K. (1985). Remembering words and how often they occurred in memory impaired patients. Memory \& Cognition, 13(6), 507-510.

Tariot, P.N. \& Weingartner, H. (1986). A psychobiological analysils of cognitive fallures. Archives of General Psychiatry, $43,1183-1188$.

Tulving, E. (1972). Episodic and semantic memory. Im: E. Tulving \& W. Donaldson (Eds.), Organization of Memory (pp. 382-403). New York: Academic Press.

Tulving. E. (1983). Elements of episodic memory. New York: Oxford University Press. 
Tulving, E. (1984). Precis of elements of episodic memory. The Behavioral and Brain Sciences, $7,223-268$.

Tulving. E. (1985). How many memory systems are there? American Psychologist, 40, 385398.

Tulving, E. (1986). What kind of a hypothesis is the distinction between episodic and semantic memory? Journal of Experimental Psychology: Learning "Memory, and Cognition, 12, 307. 311.

Tulving, E. Schacter, D.L., Mclachlan, D.R. \& Moscovitch, M. (1988). Priming of semantic autobilographical memory: A case study of retrograde amnesia. Brain and Cognition, 8, 3-20.

Uit den Boogaart, P.C. (1975). Woordfrequenties in geschreven en gesproken Nederlands. Deventer: Oosthoek, Scheltema en Molkema.

Walsh, D.A. (1982). The development of visual information processing in adulthood and old age. In: F.I.M. Craik and S. Trehub (Eds.), Aging and Cognitive Processes. (pp. 99-125). New York: Plenum Press.

Warrington, E.K. \& Weiskrantz, L. (1982). Amnesia: A disconnection syndrome? Neuropsychologia, 20, 233-248.

Watts, F.N., Morris, L. \& MacLeod, A.K. (1987). Recognition memory in depression. Jaurnal of Abnormal Psychology, 96, 273-275.

Watts, F.N. \& Sharrock, R. (1987). Cued recall in depression. British Journal of Clin/cal Psychology, 26, 149-150.

Weingartner, H. (1984). Psychobiological determinants of memory faillure. In: L.R. Squire \& $N$. Butters (Eds.). Neuropsychology of Memary (pp. 203-212). New Yark: Guilford Press.

Weingartner. H., Grafman, J., Boutelle, W., Kaye, W. \& Martin, P. (1982). Determinants of memory failures in dementia. In: S. Corkin, KK. Davis, J. Growdon, E. Usdin \& R. Wurtman (Eds.), Alzheimer's Disease. A Report of Research in Progress (Aging, Vol. 19). (pp. 171176). New York: Plaven Press.

Weingartner, $H_{.,}$Kaye, W., Smaltberg, S., Cohen, R., Ebert, M.H., Gillin, J.C. \& Gold, P. (1983). Forms of memory fallure. Science, 221, 380-382.

Weingartner, H., Kaye, W., Smallberg, S.A., Ebert, M.H."Gillin, J.C. \& Sitaram, N. (1981). Memory failures in progressive idiopathic dementia. Journal of Abnormal Psychology, 90(3). $187-196$.

Wilson, B. \& Baddeley, A. (1988). Semantic, episodic, and autobiographical memory in a postmeningitic amnesia patient. Brain and Cognition, $8,31-46$.

Wingfield, A., Aberdeen, J.S. \& Stine, E.A.L. (1991). Jounal of Gerontology: Psycholagical Sciences, 46(3), 127-129.

Witherspoon, D. \& Moscovitch, M. (1989). Stochastic independence between two implicit memory tasks. Journal of experimental Psychology: Learning, memory, and Cognition, 15(1), 22-30.

Yates, F.A. (1990). De Geheugenkunst. Amsterdam: Uitgeverij Bert Bakker.. 


\section{DANKWOORD}

Een proefschrift heeft een begin en, gelukkig, ook een eind. Allereerst een woord van

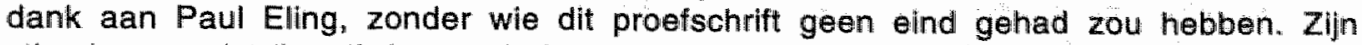
stimulans, nadat ik zelf de moed al opgegeven had is essentieel geweest. Ook dank ik de afdeling Vergelijkende en Fysiologische Psychologie te Nijmegen (o.I.v. Prof. Dr. Jo Vossen), alwaar ik het genoegen mocht smaken zowel de eerste steen van als de laatste hand aan dit boek te leggen. De gebroeders Raaijmakers, Wiljnand en Jeroen, dank ik mij in de gelegenheid gesteld te hebben het onderzoek te verrichten dat ten grondslag ligt aan dit proefschrift. Ook heb ik Jeroen's inhoudelijk commentaar zeer gewaardeerd. Jelle Jolles dank ik voor de snelle wijze waarop hij de hoofdstukken nakeek en becommentarieerde.

Ook tussen het begin en het eind zijn een aantal mensen belangrijk geweest. De collega's van de afdeling Klinische Psychologie van het Elkerliek ziekenhuis te Helmond, Gé van Woerden, Gerrit Meijer en Philine Schoondermark: zij hebben mij gedurende enkele jaren ontvangen als behoorde ik tot hun afdeling. $\mathrm{k}$ heb veel van hun geleerd, met name wat het patiëntgebonden onderzoek aangaat (de eerste patiënt zullen ook zij ongetwijfeld niet vergeten zijn). Franz Josef van der Staay, voor het mee opzetten van het eerste onderzoek en het bijeenschrapen van de apparatuur. Martin Keesen, voor het idee, de ontwikkeling en de bouw van de LED-displays. Mijn stagialies: Jan Vercoulen, Alie Stuivenga en Luce Rovers. Vooral de stage van de eerstgenoemde leverde levendige discussies op, doorspekt met een speciaal soort humor.

En, ten slotte, Tum ( $\dagger$ ), Guusje, Hendrik, Amatus en Vlam. Hun bijdrage ligt in de afleiding, in meerdere zin des woords. Treffend beschreven door Barbara Holland: "It's rare that the cat's concept of fun coincides with the human concept of work; when the human tries to do paperwork, all the cat sees is a person wasting on a sheet of paper valuable attention that might better be spent on cats;" (B. Holland, 1988: The Name of the Cat). 


\section{CURRICULUM VITAE}

Marja Abbenhuis werd op 8 april 1957 geboren te Uden. Na het gymnasium $\alpha$ volgde zij een aanvullende cursus natuur- en scheikunde aan het SAO te Utrecht. In 1976 begon zij met de studie Biologie aan de K.U. te Nijmegen, in 1977 stapte zij aldaar over naar Psychologie (oude stijl). In 1984 behaalde zij het doctoraal in de Vergelijkende en Fysiologische Psychologie met een caput in de Klinische Psychologie en als bijvak Criminologie. Tijdens de studie werkte zij part-time gedurende enkele jaren als taxichauffeur bij de Citax en gedurende een jaar als buschauffeur bij de CVD, beide te Nijmegen.

In 1985 werd zij bij de K.U. te Nijmegen aangesteld op een project getiteld: "automatische geheugenprocessen bij Dementie van het Alzheimer Type", gesubsidieerd door de SOOM. Vervolgens werkte zij tot 1990 als toegevoegd onderzoeker (NWO) bij de vakgroep Neuropsychologie en Psychobiologie van de Rijksuniversiteit Limburg te Maastricht. Het patiëntgebonden onderzoek vond plaats op de afdeling Klinische Psychologie van het Elkerliek ziekenhuis te Helmond.

Van 1989 tot 1992 vervulde zij de functie van Neuropsycholoog in een gecombineerd (somatiek en psychogeriatrie) verpleeghuis te Weert. Vanaf 1992 is zij werkzaam bij de afdeling Ouderen van de Riagg te Helmond. Sinds november 1994 is zij gedurende een jaar twee dagen per week aangesteld als projectleider van het project: "depressies bij ouderen". 